Supplementary information for:

\title{
Wetting Camphor: Multi isotopic Substitution Identifies the Complementary Roles of Hydrogen Bonding and Dispersive Forces.
}

Cristóbal Pérez ${ }^{1,2}$, Anna Krin ${ }^{1,2}$, Amanda L. Steber ${ }^{1,2,3}$, Juan C. López ${ }^{4}$, Zbigniew Kisiel ${ }^{5}$ and Melanie Schnell ${ }^{1,2,3}$

${ }^{1}$ Max Planck Institute for the Structure and Dynamics of Matter, Hamburg (Germany).

${ }^{2}$ The Center for Free-Electron Laser Science, Hamburg (Germany).

${ }^{3}$ The Hamburg Center for Ultrafast Imaging, Hamburg (Germany).

${ }^{4}$ Departamento de Quimica Fisica y Quimica Inorganica, Facultad de Ciencias, Universidad de Valladolid, Valladolid (Spain).

${ }^{5}$ Institute of Physics, Polish Academy of Sciences, Warszawa (Poland). 


\section{Table of Contents}

\section{Computational results}

Table S1

Calculated rotational constants, dipole moments, and relative energies for the normal species of complexes of camphor with water molecules.

\section{Experimental results}

\subsection{Frequencies and spectrosopic constants}

\section{$1 \mathrm{w}(\mathrm{I})$ complex}

Table S2

Spectroscopic constants for the normal and the $\mathrm{H}_{2}{ }^{18} \mathrm{O}$ substituted species of the $1 \mathrm{w}(\mathrm{I})$ complex of camphor with one water molecule.

Table S4

Measured rotational transitions for the normal species of the $1 \mathrm{w}(\mathrm{I})$ complex of camphor with one water molecule.

Table S5

Measured rotational transitions for the $\mathrm{H}_{2}{ }^{18} \mathrm{O}$ substituted species of the $1 \mathrm{w}(\mathrm{I})$ complex of camphor with one water molecule.

\section{$1 \mathrm{w}(\mathrm{II})$ complex}

Table S6

Spectroscopic constants for the normal and the $\mathrm{H}_{2}{ }^{18} \mathrm{O}$ substituted species of the $1 \mathrm{w}$ (II) complex of camphor with one water molecule.

Table S8

Measured rotational transitions for the normal species of the $1 \mathrm{w}(\mathrm{II})$ complex of camphor with one water molecule.

Table S9

Measured rotational transitions for the $\mathrm{H}_{2}{ }^{18} \mathrm{O}$ substituted species of the $1 \mathrm{w}(\mathrm{II})$ complex of camphor with one water molecule.

\section{2w(I) complex}

Table S10

Spectroscopic constants for the normal and the $\mathrm{H}_{2}{ }^{18} \mathrm{O}$ substituted species of the $2 \mathrm{w}(\mathrm{I})$ complex of camphor with two water molecules.

Table S12

Measured rotational transitions for the normal species of the $2 \mathrm{w}(\mathrm{I})$ complex of camphor with two water molecules.

Table S13-15 Measured rotational transitions for the $\mathrm{H}_{2}{ }^{18} \mathrm{O}$ substituted species of the $2 \mathrm{w}(\mathrm{I})$ complex of camphor with two water molecules.

$2 \mathrm{w}(\mathrm{II})$ complex

Table S16

Spectroscopic constants for the normal and the $\mathrm{H}_{2}{ }^{18} \mathrm{O}$ substituted species of the $2 \mathrm{w}$ (II) complex of camphor with two water molecules. 
Table S18

Table S19-21

\section{3w complex}

Table S22

Table S24

Table S25-31
Measured rotational transitions for the normal species of the $2 \mathrm{w}$ (II) complex of camphor with two water molecules.

Measured rotational transitions for the $\mathrm{H}_{2}{ }^{18} \mathrm{O}$ substituted species of the $2 \mathrm{w}$ (II) complex of camphor with two water molecules.

Spectroscopic constants for the normal and the $\mathrm{H}_{2}{ }^{18} \mathrm{O}$ substituted species of the $3 \mathrm{w}$ complex of camphor with three water molecules.

Measured rotational transitions for the normal species of the $3 \mathrm{w}$ complex of camphor with three water molecules.

Measured rotational transitions for the $\mathrm{H}_{2}{ }^{18} \mathrm{O}$ substituted species of the $3 \mathrm{w}$ complex of camphor with three water molecules.

\subsection{Structural analysis}

\section{$1 \mathrm{w}(\mathrm{I})$ complex}

Figure S1

Table S3

Table S32

\section{$1 \mathrm{w}(\mathrm{II})$ complex}

Figure S2

Table S7

Table S33

\section{$2 \mathrm{w}(\mathrm{I})$ complex}

Figure S3

Table S11

Table S34

\section{2w(II) complex}

Figure S4
Calculated structure for the $1 \mathrm{w}(\mathrm{I})$ complex of camphor with one water molecule.

Experimental coordinates of the water oxygen atom in the $1 \mathrm{w}(\mathrm{I})$ complex with one water.

The abbreviated results of the $\mathrm{r}_{0}$ geometry of $1 \mathrm{w}(\mathrm{I})$ complex with one water.

Calculated structure for the $1 \mathrm{w}(\mathrm{II})$ complex of camphor with one water. molecule.

Experimental coordinates of the water oxygen atom in the $1 \mathrm{w}$ (II) complex with one water.

The abbreviated results of the $\mathrm{r}_{0}$ geometry of $1 \mathrm{w}(\mathrm{II})$ complex with one water.

Calculated structures for the camphor complex $2 \mathrm{w}(\mathrm{I})$ with two water molecules.

Experimental coordinates of the water oxygen atom in the $2 \mathrm{w}(\mathrm{I})$ complex with two water molecules.

The abbreviated results of the $\mathrm{r}_{0}$ geometry of $2 \mathrm{w}(\mathrm{I})$ complex with two water molecules.

Calculated structures for the camphor complex $2 \mathrm{w}$ (II) with two water molecules. 
Table S17

Table S35

3w complex

Figure S5

Table S23

Table S36
Experimental coordinates of the water oxygen atom in the $2 \mathrm{w}$ (II) complex with two water molecules.

The abbreviated results of the $\mathrm{r}_{0}$ geometry of $2 \mathrm{w}$ (II) complex with two water molecules.

Calculated structure for the camphor complex with three water molecules.

Experimental coordinates of the water oxygen atom in the $3 \mathrm{w}$ complex with three water molecules.

The abbreviated results of the $\mathrm{r}_{0}$ geometry of $3 \mathrm{w}$ complex with three water molecules. 
Table S1: Calculated rotational constants, dipole moments, and relative energies for the normal species of complexes of camphor with water molecules.

\begin{tabular}{|c|c|c|c|c|c|c|}
\hline & Calc $^{a}$ & Calc $^{b}$ & $\mathrm{Calc}^{c}$ & $\mathrm{Calc}^{a}$ & $\mathrm{Calc}^{b}$ & $\mathrm{Calc}^{c}$ \\
\hline & \multicolumn{3}{|c|}{$1 \mathrm{w}(\mathrm{I})$} & \multicolumn{3}{|c|}{$1 \mathrm{w}(\mathrm{II})$} \\
\hline$A(\mathrm{MHz})$ & 1326 & 1345 & 1348 & 1339 & 1325 & 1339 \\
\hline$B(\mathrm{MHz})$ & 674 & 686 & 669 & 696 & 732 & 700 \\
\hline$C(\mathrm{MHz})$ & 655 & 656 & 642 & 677 & 720 & 682 \\
\hline$\left|\mu_{a}\right|(\mathrm{D})$ & 2.61 & 2.61 & 3.07 & 2.72 & 2.31 & 3.01 \\
\hline$\left|\mu_{b}\right|(\mathrm{D})$ & 0.06 & 0.20 & 0.24 & 0.79 & 0.70 & 0.75 \\
\hline$\left|\mu_{c}\right|(\mathrm{D})$ & 0.24 & 0.13 & 0.17 & 0.05 & 0.47 & 0.18 \\
\hline \multirow[t]{2}{*}{$\Delta E(\mathrm{~kJ} / \mathrm{mol})$} & 0.0 & 0.0 & 0.0 & 0.63 & 1.43 & 1.48 \\
\hline & \multicolumn{3}{|c|}{$2 \mathrm{w}(\mathrm{I})$} & \multicolumn{3}{|c|}{$2 w(I I)$} \\
\hline$A(\mathrm{MHz})$ & 1077 & 1083 & 1070 & 1149 & 1150 & 1142 \\
\hline$B(\mathrm{MHz})$ & 518 & 530 & 521 & 534 & 557 & 535 \\
\hline$C(\mathrm{MHz})$ & 476 & 489 & 479 & 484 & 509 & 483 \\
\hline$\left|\mu_{a}\right|(\mathrm{D})$ & 1.17 & 1.11 & 1.77 & 0.74 & 0.42 & 1.25 \\
\hline$\left|\mu_{b}\right|(\mathrm{D})$ & 0.06 & 0.12 & 0.15 & 0.27 & 0.19 & 0.53 \\
\hline$\left|\mu_{c}\right|(\mathrm{D})$ & 0.12 & 1.24 & 0.62 & 0.13 & 0.08 & 0.14 \\
\hline \multirow[t]{2}{*}{$\Delta E(\mathrm{~kJ} / \mathrm{mol})$} & 0.0 & 1.87 & 0.0 & 0.39 & 0.0 & 0.51 \\
\hline & \multicolumn{3}{|c|}{$3 w$} & & & \\
\hline$A(\mathrm{MHz})$ & 917 & - & 906 & & & \\
\hline$B(\mathrm{MHz})$ & 398 & - & 407 & & & \\
\hline$C(\mathrm{MHz})$ & 354 & - & 360 & & & \\
\hline$\left|\mu_{a}\right|(\mathrm{D})$ & 1.06 & - & 1.43 & & & \\
\hline$\left|\mu_{b}\right|(\mathrm{D})$ & 0.48 & - & 0.16 & & & \\
\hline$\left|\mu_{c}\right|(\mathrm{D})$ & 0.76 & - & 0.83 & & & \\
\hline$\Delta E(\mathrm{~kJ} / \mathrm{mol})$ & 0.0 & - & 0.0 & & & \\
\hline
\end{tabular}

${ }^{a} \mathrm{MP} 2 / 6-311++\mathrm{G}(\mathrm{d}, \mathrm{p})$ calculation.

${ }^{b} \mathrm{M} 06-2 \mathrm{X} / 6-311++\mathrm{G}(\mathrm{d}, \mathrm{p})$ calculation.

${ }^{c}$ B3LYP-D3/aug-cc-pVTZ calculation. 


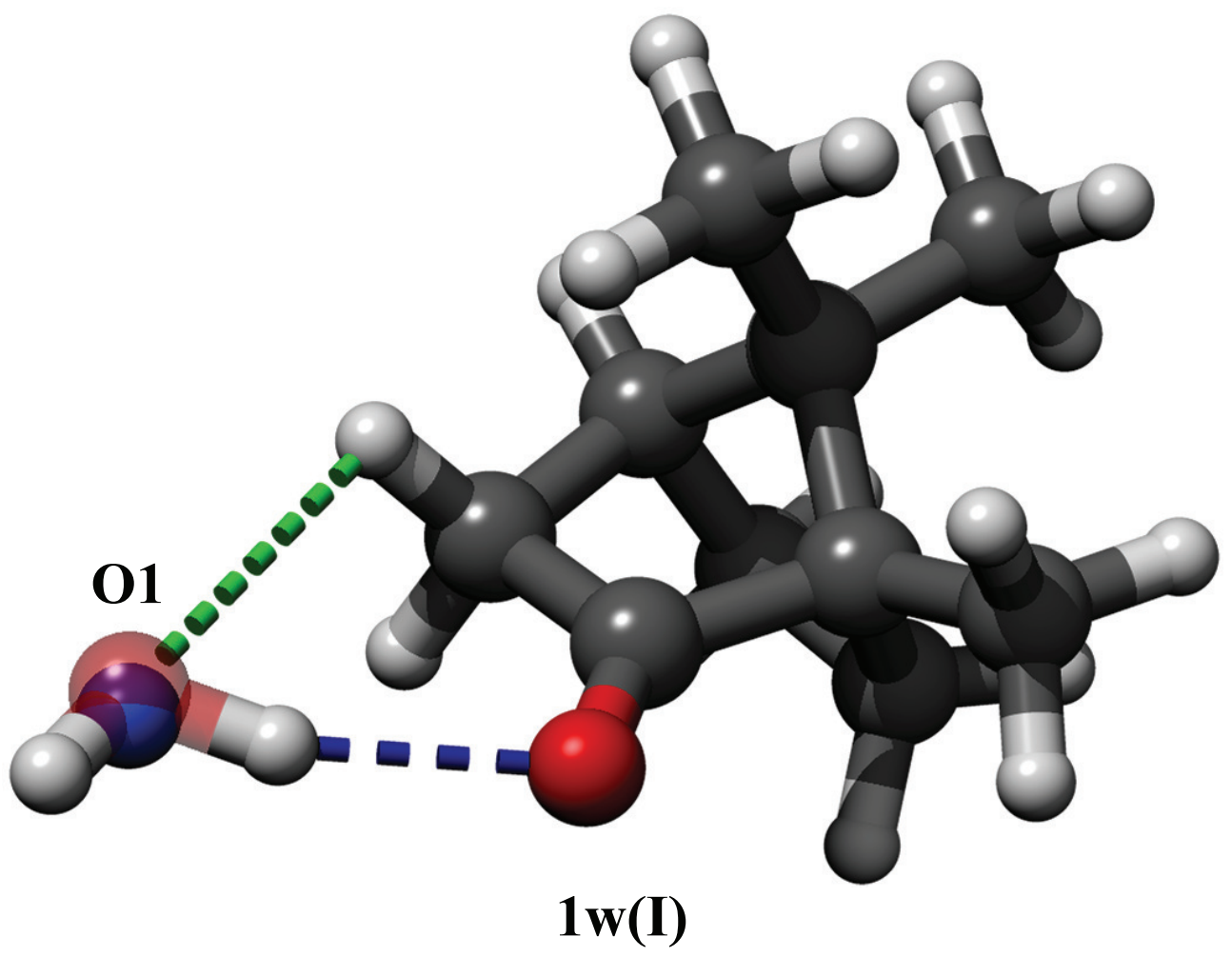

Figure S1: Calculated structure (MP2/6-311++G(d,p)) for the $1 \mathrm{w}(\mathrm{I})$ complex of camphor with one water molecule. Oxygen atom numbering used for the $\mathrm{H}_{2}{ }^{18} \mathrm{O}$ isotopologue of the complex is shown. The blue/purple sphere represents the experimental oxygen position from the Kraitchman analysis. Strong hydrogen bond is depicted in blue, dispersive interaction is shown in green. 
Table S2: Experimentally determined rotational and quartic centrifugal distortion constants for the normal and the $\mathrm{H}_{2}{ }^{18} \mathrm{O}$ substituted species of the $1 \mathrm{w}(\mathrm{I})$ complex of camphor with one water molecule (see Figure S1 for oxygen atom numbering).

\begin{tabular}{lcc}
\hline & normal & ${ }^{18} \mathrm{O} 1$ \\
\hline$A(\mathrm{MHz})$ & $1344.654(66)$ & $1343.093(59)$ \\
$B(\mathrm{MHz})$ & $662.98812(73)$ & $635.15575(25)$ \\
$C(\mathrm{MHz})$ & $637.90564(72)$ & $611.99682(24)$ \\
$\Delta_{J}(\mathrm{kHz})$ & $0.1482(28)$ & $a$ \\
$\Delta_{J K}(\mathrm{kHz})$ & $2.163(16)$ & $a$ \\
$\delta_{J}(\mathrm{kHz})$ & $-0.0197(47)$ & $a$ \\
$N_{\text {lines }}$ & 38 & 41 \\
$\sigma(\mathrm{kHz})$ & 4.64 & 4.42 \\
& & \\
$P_{a}\left(\mathrm{u} \AA^{2}\right)^{c}$ & $589.339(9)$ & $622.592(8)$ \\
$P_{b}\left(\mathrm{u} \AA^{2}\right)$ & $202.908(9)$ & $203.195(8)$ \\
$P_{c}\left(\mathrm{u} \AA^{2}\right)$ & $172.935(9)$ & $173.085(8)$ \\
\hline
\end{tabular}

${ }^{a}$ Kept fixed at the value for the normal species.

${ }^{b}$ The number of fitted lines.

${ }^{c}$ Planar moment of inertia, $P_{a}=\sum\left(m_{i} a_{i}^{2}\right)=\frac{1}{2}\left(I_{b}+I_{c}-I_{a}\right)$ etc. 
Table S3: Experimental coordinates $(\AA)$ of the water oxygen atom $\mathrm{O} 1$ in the $1 \mathrm{w}(\mathrm{I})$ complex of camphor with one water molecule, compared with the results from ab initio calculations.

\begin{tabular}{rlrrrr}
\hline & \multicolumn{1}{c}{$r_{\mathrm{s}}{ }^{a}$} & \multicolumn{1}{c}{$r_{0}{ }^{b}$} & \multicolumn{1}{c}{ Calc $^{c}$} & Calc $^{d}$ & \multicolumn{1}{c}{ Calc $^{e}$} \\
\hline & & & & & \\
$b$ & $4.0949(9)$ & $4.0932(2)$ & 4.0101 & 3.9648 & 4.0549 \\
$b$ & $0.3956(94)$ & $-0.4393(5)$ & -0.4899 & -0.4629 & -0.4436 \\
$c$ & $0.2872(130)$ & $0.3421(4)$ & 0.3795 & 0.3308 & 0.3369 \\
\hline
\end{tabular}

${ }^{a}$ Determined using Kraitchman equations, which result in only the absolute value, while uncertainties were estimated with the Costain criterion.

${ }^{b}$ Obtained from the least squares $r_{0}$ fit where the cited uncertainties only reflect those in the explicit parameters of the fit.

${ }^{c} \mathrm{MP} 2 / 6-311++\mathrm{G}(\mathrm{d}, \mathrm{p})$ calculation.

${ }^{d} \mathrm{M} 06-2 \mathrm{X} / 6-311++\mathrm{G}(\mathrm{d}, \mathrm{p})$ calculation.

${ }^{r}$ B3LYP-D3/aug-cc-pVTZ calculation. 
Table S4: Measured rotational transitions ( $\left.\nu_{\text {obs }}\right)$ for the $1 \mathrm{w}(\mathrm{I})$ complex of camphor with one water molecule (main isotopologue), and the residuals $\left(\nu_{\mathrm{obs}}-\nu_{\text {calc }}\right)$ for the fit reported in Table 1.

\begin{tabular}{|c|c|c|c|c|c|c|c|c|}
\hline$J^{\prime}$ & $K_{a}^{\prime}$ & $K_{c}^{\prime}$ & $\leftarrow$ & $J^{\prime \prime}$ & $K_{a}^{\prime \prime}$ & $K_{c}^{\prime \prime}$ & $\nu_{\mathrm{obs}}(\mathrm{MHz})$ & $\nu_{\mathrm{obs}}-\nu_{\mathrm{calc}}$ \\
\hline 2 & 1 & 2 & $\leftarrow$ & 1 & 1 & 1 & 2576.6981 & 0.0070 \\
\hline 2 & 0 & 2 & $\leftarrow$ & 1 & 0 & 1 & 2601.1025 & -0.0008 \\
\hline 2 & 1 & 1 & $\leftarrow$ & 1 & 1 & 0 & 2626.8608 & 0.0036 \\
\hline 3 & 1 & 3 & $\leftarrow$ & 2 & 1 & 2 & 3864.6081 & 0.0008 \\
\hline 3 & 0 & 3 & $\leftarrow$ & 2 & 0 & 2 & 3899.9447 & -0.0054 \\
\hline 3 & 2 & 2 & $\leftarrow$ & 2 & 2 & 1 & 3902.6084 & -0.0050 \\
\hline 3 & 2 & 1 & $\leftarrow$ & 2 & 2 & 0 & 3905.3278 & -0.0008 \\
\hline 3 & 1 & 2 & $\leftarrow$ & 2 & 1 & 1 & 3939.8460 & -0.0016 \\
\hline 4 & 1 & 4 & $\leftarrow$ & 3 & 1 & 3 & 5152.0229 & -0.0015 \\
\hline 4 & 0 & 4 & $\leftarrow$ & 3 & 0 & 3 & 5196.7766 & 0.0063 \\
\hline 4 & 2 & 3 & $\leftarrow$ & 3 & 2 & 2 & 5202.9433 & 0.0041 \\
\hline 4 & 2 & 2 & $\leftarrow$ & 3 & 2 & 1 & 5209.7089 & 0.0030 \\
\hline 4 & 3 & 2 & $\leftarrow$ & 3 & 3 & 1 & 5204.7368 & -0.0045 \\
\hline 4 & 3 & 1 & $\leftarrow$ & 3 & 3 & 0 & 5204.7368 & -0.0045 \\
\hline 4 & 1 & 3 & $\leftarrow$ & 3 & 1 & 2 & 5252.2937 & -0.0016 \\
\hline 5 & 1 & 5 & $\leftarrow$ & 4 & 1 & 4 & 6438.7915 & -0.0102 \\
\hline 5 & 0 & 5 & $\leftarrow$ & 4 & 0 & 4 & 6490.9550 & 0.0069 \\
\hline 5 & 2 & 4 & $\leftarrow$ & 4 & 2 & 3 & 6502.7993 & 0.0015 \\
\hline 5 & 2 & 3 & $\leftarrow$ & 4 & 2 & 2 & 6516.2413 & -0.0018 \\
\hline 5 & 3 & 3 & $\leftarrow$ & 4 & 3 & 2 & 6506.4153 & -0.0026 \\
\hline 5 & 3 & 2 & $\leftarrow$ & 4 & 3 & 1 & 6506.6573 & -0.0021 \\
\hline 5 & 4 & 2 & $\leftarrow$ & 4 & 4 & 1 & 6505.5665 & 0.0068 \\
\hline 5 & 4 & 1 & $\leftarrow$ & 4 & 4 & 0 & 6505.5665 & 0.0068 \\
\hline 5 & 1 & 4 & $\leftarrow$ & 4 & 1 & 3 & 6563.9913 & -0.0011 \\
\hline 6 & 1 & 6 & $\leftarrow$ & 5 & 1 & 5 & 7724.8225 & 0.0023 \\
\hline 6 & 0 & 6 & $\leftarrow$ & 5 & 0 & 5 & 7781.9798 & 0.0074 \\
\hline 6 & 2 & 5 & $\leftarrow$ & 5 & 2 & 4 & 7802.0701 & -0.0029 \\
\hline 6 & 2 & 4 & $\leftarrow$ & 5 & 2 & 3 & 7825.3288 & -0.0005 \\
\hline 6 & 3 & 4 & $\leftarrow$ & 5 & 3 & 3 & 7808.4239 & -0.0008 \\
\hline 6 & 3 & 3 & $\leftarrow$ & 5 & 3 & 2 & 7809.0619 & -0.0058 \\
\hline 6 & 4 & 3 & $\leftarrow$ & 5 & 4 & 2 & 7807.1291 & -0.0027 \\
\hline 6 & 4 & 2 & $\leftarrow$ & 5 & 4 & 1 & 7807.1291 & -0.0027 \\
\hline 6 & 1 & 5 & $\leftarrow$ & 5 & 1 & 4 & 7874.7107 & 0.0046 \\
\hline 7 & 1 & 7 & $\leftarrow$ & 6 & 1 & 6 & 9009.9892 & 0.0036 \\
\hline 7 & 0 & 7 & $\leftarrow$ & 6 & 0 & 6 & 9069.5013 & -0.0093 \\
\hline 7 & 2 & 6 & $\leftarrow$ & 6 & 2 & 5 & 9100.6523 & 0.0023 \\
\hline 7 & 2 & 5 & $\leftarrow$ & 6 & 2 & 4 & 9137.1732 & 0.0002 \\
\hline 7 & 3 & 5 & $\leftarrow$ & 6 & 3 & 4 & 9110.7320 & 0.0036 \\
\hline 7 & 3 & 4 & $\leftarrow$ & 6 & 3 & 3 & 9112.1719 & 0.0007 \\
\hline 7 & 4 & 4 & $\leftarrow$ & 6 & 4 & 3 & 9108.9571 & 0.0015 \\
\hline 7 & 4 & 3 & $\leftarrow$ & 6 & 4 & 2 & 9108.9571 & 0.0015 \\
\hline 7 & 1 & 6 & $\leftarrow$ & 6 & 1 & 5 & 9184.1712 & -0.0030 \\
\hline & & & & & & & RMS & $4.644 \mathrm{kHz}$ \\
\hline
\end{tabular}


Table S5: Measured rotational transitions $\left(\nu_{\mathrm{obs}}\right)$ for the $1 \mathrm{w}(\mathrm{I}){ }^{18} \mathrm{O} 1$ complex of camphor with one water molecule, and the residuals $\left(\nu_{\mathrm{obs}}-\nu_{\text {calc }}\right)$ for the fit reported in Table S2 (see Figure S1 for oxygen atom numbering).

\begin{tabular}{|c|c|c|c|c|c|c|c|c|}
\hline$J^{\prime}$ & $K_{a}^{\prime}$ & $K_{c}^{\prime}$ & $\leftarrow$ & $J^{\prime \prime}$ & $K_{a}^{\prime \prime}$ & $K_{c}^{\prime \prime}$ & $\nu_{\mathrm{obs}}(\mathrm{MHz})$ & $\nu_{\mathrm{obs}}-\nu_{\mathrm{calc}}$ \\
\hline 2 & 1 & 2 & $\leftarrow$ & 1 & 1 & 1 & 2471.1342 & 0.0020 \\
\hline 2 & 0 & 2 & $\leftarrow$ & 1 & 0 & 1 & 2493.7402 & -0.0012 \\
\hline 2 & 1 & 1 & $\leftarrow$ & 1 & 1 & 0 & 2517.4551 & 0.0038 \\
\hline 3 & 1 & 3 & $\leftarrow$ & 2 & 1 & 2 & 3706.3369 & -0.0061 \\
\hline 3 & 0 & 3 & $\leftarrow$ & 2 & 0 & 2 & 3739.2044 & -0.0034 \\
\hline 3 & 2 & 2 & $\leftarrow$ & 2 & 2 & 1 & 3741.3850 & -0.0048 \\
\hline 3 & 2 & 1 & $\leftarrow$ & 2 & 2 & 0 & 3743.6157 & -0.0081 \\
\hline 3 & 1 & 2 & $\leftarrow$ & 2 & 1 & 1 & 3775.8142 & -0.0014 \\
\hline 4 & 1 & 4 & $\leftarrow$ & 3 & 1 & 3 & 4941.1357 & -0.0033 \\
\hline 4 & 0 & 4 & $\leftarrow$ & 3 & 0 & 3 & 4983.0007 & -0.0004 \\
\hline 4 & 2 & 3 & $\leftarrow$ & 3 & 2 & 2 & 4988.0686 & 0.0003 \\
\hline 4 & 2 & 2 & $\leftarrow$ & 3 & 2 & 1 & 4993.6408 & 0.0014 \\
\hline 4 & 3 & 2 & $\leftarrow$ & 3 & 3 & 1 & 4989.5490 & 0.0139 \\
\hline 4 & 3 & 1 & $\leftarrow$ & 3 & 3 & 0 & 4989.5490 & 0.0139 \\
\hline 4 & 1 & 3 & $\leftarrow$ & 3 & 1 & 2 & 5033.7332 & -0.0009 \\
\hline 5 & 1 & 5 & $\leftarrow$ & 4 & 1 & 4 & 6175.4018 & 0.0009 \\
\hline 5 & 0 & 5 & $\leftarrow$ & 4 & 0 & 4 & 6224.6009 & -0.0022 \\
\hline 5 & 2 & 4 & $\leftarrow$ & 4 & 2 & 3 & 6234.3651 & 0.0053 \\
\hline 5 & 2 & 3 & $\leftarrow$ & 4 & 2 & 2 & 6245.4492 & 0.0046 \\
\hline 5 & 3 & 3 & $\leftarrow$ & 4 & 3 & 2 & 6237.3306 & 0.0016 \\
\hline 5 & 3 & 2 & $\leftarrow$ & 4 & 3 & 1 & 6237.5069 & 0.0009 \\
\hline 5 & 1 & 4 & $\leftarrow$ & 4 & 1 & 3 & 6291.0404 & 0.0022 \\
\hline 5 & 4 & 1 & $\leftarrow$ & 4 & 4 & 0 & 6236.5842 & -0.0018 \\
\hline 6 & 1 & 6 & $\leftarrow$ & 5 & 1 & 5 & 7409.0222 & -0.0029 \\
\hline 6 & 0 & 6 & $\leftarrow$ & 5 & 0 & 5 & 7463.5624 & -0.0022 \\
\hline 6 & 2 & 5 & $\leftarrow$ & 5 & 2 & 4 & 7480.1642 & -0.0039 \\
\hline 6 & 2 & 4 & $\leftarrow$ & 5 & 2 & 3 & 7499.3922 & 0.0051 \\
\hline 6 & 3 & 4 & $\leftarrow$ & 5 & 3 & 3 & 7485.3947 & -0.0032 \\
\hline 6 & 3 & 3 & $\leftarrow$ & 5 & 3 & 2 & 7485.8611 & -0.0082 \\
\hline 6 & 4 & 3 & $\leftarrow$ & 5 & 4 & 2 & 7484.2647 & -0.0095 \\
\hline 6 & 4 & 2 & $\leftarrow$ & 5 & 4 & 1 & 7484.2647 & -0.0095 \\
\hline 6 & 1 & 5 & $\leftarrow$ & 5 & 1 & 4 & 7547.5419 & 0.0006 \\
\hline 7 & 1 & 7 & $\leftarrow$ & 6 & 1 & 6 & 8641.9274 & 0.0002 \\
\hline 7 & 0 & 7 & $\leftarrow$ & 6 & 0 & 6 & 8699.5579 & -0.0001 \\
\hline 7 & 2 & 6 & $\leftarrow$ & 6 & 2 & 5 & 8725.4000 & 0.0024 \\
\hline 7 & 2 & 5 & $\leftarrow$ & 6 & 2 & 4 & 8755.6922 & 0.0006 \\
\hline 7 & 3 & 5 & $\leftarrow$ & 6 & 3 & 4 & 8733.7262 & 0.0026 \\
\hline 7 & 3 & 4 & $\leftarrow$ & 6 & 3 & 3 & 8734.7852 & 0.0031 \\
\hline 7 & 4 & 4 & $\leftarrow$ & 6 & 4 & 3 & 8732.1616 & -0.0044 \\
\hline 7 & 4 & 3 & $\leftarrow$ & 6 & 4 & 2 & 8732.1616 & -0.0044 \\
\hline 7 & 1 & 6 & $\leftarrow$ & 6 & 1 & 5 & 8803.0335 & -0.0017 \\
\hline 8 & 1 & 8 & $\leftarrow$ & 7 & 1 & 7 & 9874.0481 & 0.0050 \\
\hline 8 & 0 & 8 & $\leftarrow$ & 7 & 0 & 7 & 9932.4379 & 0.0034 \\
\hline \multirow[t]{2}{*}{8} & 2 & 7 & $\leftarrow$ & 7 & 2 & 6 & 9969.9565 & 0.0024 \\
\hline & & & & & & & RMS & $4.415 \mathrm{kHz}$ \\
\hline
\end{tabular}




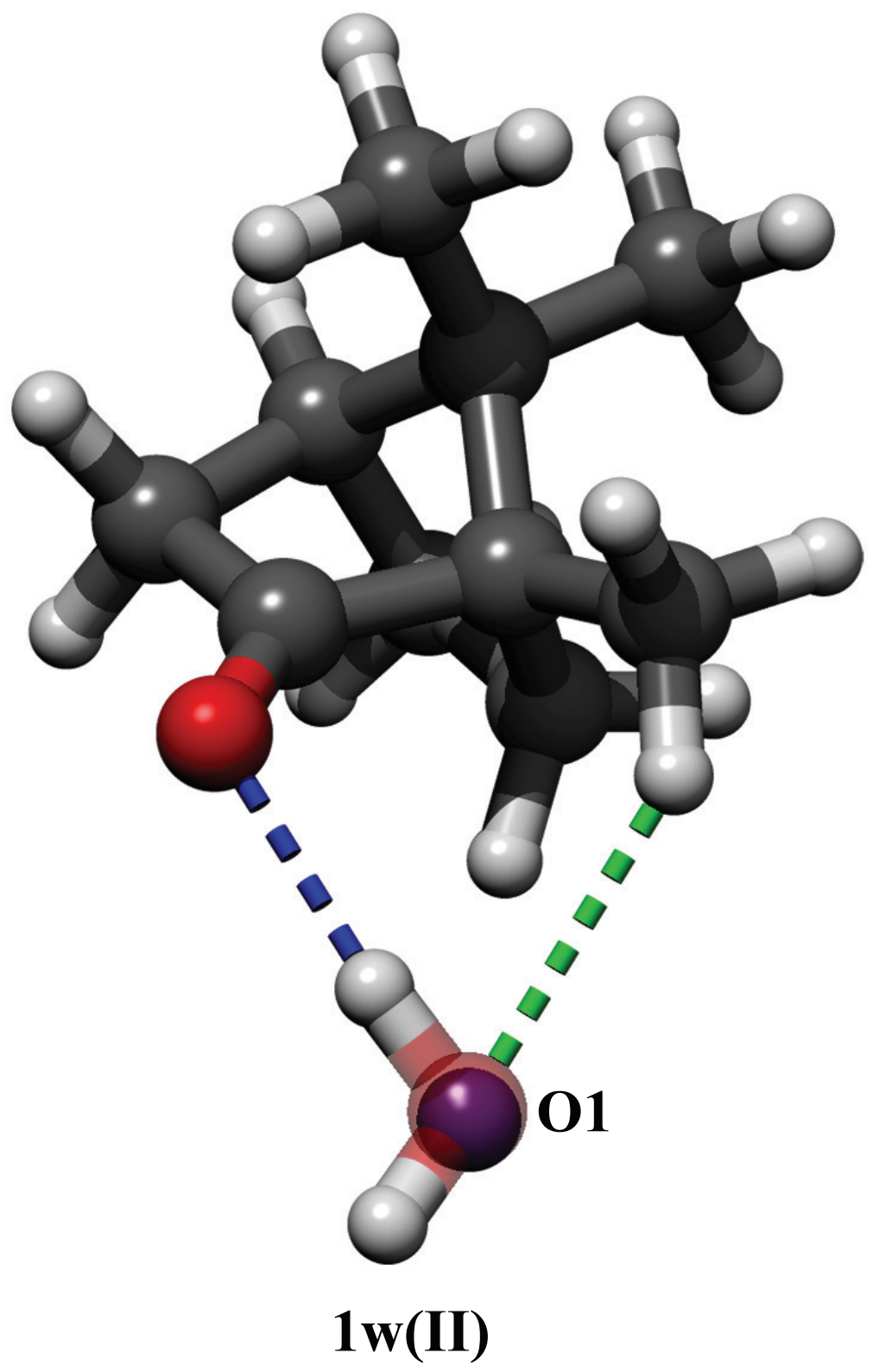

Figure S2: Calculated structure (MP2/6-311++G(d,p)) for the $1 \mathrm{w}(\mathrm{II})$ complex of camphor with one water molecule. Oxygen atom numbering used for the $\mathrm{H}_{2}{ }^{18} \mathrm{O}$ isotopologue of the complex is shown. The purple sphere represents the experimental oxygen position from the Kraitchman analysis. Strong hydrogen bond is depicted in blue, dispersive interaction is shown in green. 
Table S6: Experimentally determined rotational and quartic centrifugal distortion constants for the normal and the $\mathrm{H}_{2}{ }^{18} \mathrm{O}$ substituted species of the $1 \mathrm{w}(\mathrm{II})$ complex of camphor with one water molecule (see Figure S2 for oxygen atom numbering).

\begin{tabular}{lcc}
\hline & normal & ${ }^{18} \mathrm{O} 1$ \\
\hline$A(\mathrm{MHz})$ & $1339.83(15)$ & $1336.73(19)$ \\
$B(\mathrm{MHz})$ & $689.03465(94)$ & $662.27538(33)$ \\
$C(\mathrm{MHz})$ & $672.95812(73)$ & $647.64338(33)$ \\
$\Delta_{J}(\mathrm{kHz})$ & $0.7309(44)$ & $a$ \\
$\Delta_{J K}(\mathrm{kHz})$ & $1.273(18)$ & $a$ \\
$\delta_{J}(\mathrm{kHz})$ & $-0.1234(61)$ & $a$ \\
$N_{\text {lines }}$ & 28 & 28 \\
$\sigma(\mathrm{kHz})$ & 3.96 & 4.96 \\
& & \\
$P_{a}\left(\mathrm{u} \AA^{2}\right)$ & $553.62(2)$ & $582.68(3)$ \\
$P_{b}\left(\mathrm{u} \AA^{2}\right)$ & $197.36(2)$ & $197.66(3)$ \\
$P_{c}\left(\mathrm{u} \AA^{2}\right)$ & $179.84(2)$ & $180.42(3)$ \\
\hline
\end{tabular}

${ }^{a}$ Kept fixed at the value for the normal species. 
Table S7: Experimental coordinates $(\AA)$ of the water oxygen atom in the $1 \mathrm{w}(\mathrm{II})$ complex of camphor with one water molecule, compared with the results from ab initio calculations.

\begin{tabular}{rlrrrr}
\hline & \multicolumn{1}{c}{$r_{\mathrm{s}}{ }^{a}$} & \multicolumn{1}{c}{$r_{0}{ }^{b}$} & \multicolumn{1}{c}{ Calc $^{c}$} & \multicolumn{1}{c}{ Calc $^{d}$} & \multicolumn{1}{c}{ Calc $^{e}$} \\
\hline & & & & & \\
$a$ & $3.8254(23)$ & $-3.8706(12)$ & -3.8397 & -3.5727 & -3.7916 \\
$b$ & $0.3957(231)$ & $0.4048(52)$ & 0.4392 & 0.5084 & 0.4478 \\
$c$ & $0.5656(169)$ & $0.4607(30)$ & 0.4412 & 0.4707 & 0.4438 \\
\hline
\end{tabular}

${ }^{a}$ Determined using Kraitchman equations, which result in only the absolute value, while uncertainties were estimated with the Costain criterion.

${ }^{b}$ Obtained from the least squares $r_{0}$ fit where the cited uncertainties only reflect those in the explicit parameters of the fit.

${ }^{c} \mathrm{MP} 2 / 6-311++\mathrm{G}(\mathrm{d}, \mathrm{p})$ calculation.

${ }^{d} \mathrm{M} 06-2 \mathrm{X} / 6-311++\mathrm{G}(\mathrm{d}, \mathrm{p})$ calculation.

${ }^{r}$ B3LYP-D3/aug-cc-pVTZ calculation. 
Table S8: Measured rotational transitions ( $\left.\nu_{\mathrm{obs}}\right)$ for the $1 \mathrm{w}(\mathrm{II})$ complex of camphor with one water molecule (main isotopologue), and the residuals $\left(\nu_{\mathrm{obs}}-\nu_{\text {calc }}\right)$ for the fit reported in Table 1.

\begin{tabular}{ccccccccc}
\hline$J^{\prime}$ & $K_{a}^{\prime}$ & $K_{c}^{\prime}$ & $\leftarrow$ & $J^{\prime \prime}$ & $K_{a}^{\prime \prime}$ & $K_{c}^{\prime \prime}$ & $\nu_{\mathrm{obs}}(\mathrm{MHz})$ & $\nu_{\mathrm{obs}}-\nu_{\text {calc }}$ \\
\hline & & & & & & & & \\
2 & 1 & 2 & $\leftarrow$ & 1 & 1 & 1 & 2707.8784 & 0.0018 \\
2 & 0 & 2 & $\leftarrow$ & 1 & 0 & 1 & 2723.6693 & 0.0014 \\
& & & & & & & & \\
3 & 1 & 3 & $\leftarrow$ & 2 & 1 & 2 & 4061.5810 & -0.0003 \\
3 & 0 & 3 & $\leftarrow$ & 2 & 0 & 2 & 4084.7159 & -0.0064 \\
& & & & & & & & \\
4 & 1 & 4 & $\leftarrow$ & 3 & 1 & 3 & 5415.0091 & -0.0009 \\
4 & 0 & 4 & $\leftarrow$ & 3 & 0 & 3 & 5444.8386 & -0.0051 \\
4 & 2 & 3 & $\leftarrow$ & 3 & 2 & 2 & 5447.5158 & 0.0016 \\
4 & 2 & 2 & $\leftarrow$ & 3 & 2 & 1 & 5450.4525 & -0.0020 \\
4 & 3 & 2 & $\leftarrow$ & 3 & 3 & 1 & 5448.2886 & 0.0070 \\
4 & 3 & 1 & $\leftarrow$ & 3 & 3 & 0 & 5448.2886 & 0.0070 \\
4 & 1 & 3 & $\leftarrow$ & 3 & 1 & 2 & 5479.3615 & 0.0025 \\
& & & & & & & & \\
5 & 1 & 5 & $\leftarrow$ & 4 & 1 & 4 & 6768.0796 & 0.0020 \\
5 & 0 & 5 & $\leftarrow$ & 4 & 0 & 4 & 6803.7286 & -0.0018 \\
5 & 2 & 4 & $\leftarrow$ & 4 & 2 & 3 & 6808.8930 & 0.0006 \\
5 & 2 & 3 & $\leftarrow$ & 4 & 2 & 2 & 6814.7631 & 0.0031 \\
5 & 3 & 3 & $\leftarrow$ & 4 & 3 & 2 & 6810.4655 & 0.0030 \\
5 & 3 & 2 & $\leftarrow$ & 4 & 3 & 1 & 6810.5265 & -0.0068 \\
5 & 4 & 1 & $\leftarrow$ & 4 & 4 & 0 & 6810.0488 & -0.0013 \\
5 & 1 & 4 & $\leftarrow$ & 4 & 1 & 3 & 6848.5133 & 0.0004 \\
& & & & & & & & \\
6 & 1 & 6 & $\leftarrow$ & 5 & 1 & 5 & 8120.7090 & 0.0035 \\
6 & 0 & 6 & $\leftarrow$ & 5 & 0 & 5 & 8161.1010 & -0.0004 \\
6 & 2 & 5 & $\leftarrow$ & 5 & 2 & 4 & 8169.9427 & 0.0064 \\
6 & 2 & 4 & $\leftarrow$ & 5 & 2 & 3 & 8180.1588 & -0.0001 \\
6 & 3 & 4 & $\leftarrow$ & 5 & 3 & 3 & 8172.7142 & -0.0038 \\
6 & 3 & 3 & $\leftarrow$ & 5 & 3 & 2 & 8172.9000 & -0.0070 \\
6 & 4 & 3 & $\leftarrow$ & 5 & 4 & 2 & 8172.0881 & 0.0037 \\
6 & 4 & 2 & $\leftarrow$ & 5 & 4 & 1 & 8172.0881 & 0.0037 \\
6 & 1 & 5 & $\leftarrow$ & 5 & 1 & 4 & 8217.1889 & 0.0000 \\
& & & & & & & & \\
7 & 1 & 7 & $\leftarrow$ & 6 & 1 & 6 & 9472.8193 & -0.0025 \\
7 & 0 & 7 & $\leftarrow$ & 6 & 0 & 6 & 9516.7133 & 0.0012 \\
& & & & & & & & \\
& & & & & & & $\mathrm{RMS}$ & $3.961 \mathrm{kHz}$ \\
\hline & & & & & & & &
\end{tabular}


Table S9: Measured rotational transitions $\left(\nu_{\mathrm{obs}}\right)$ for the $1 \mathrm{w}(\mathrm{II})-{ }^{18} \mathrm{O} 1$ complex of camphor with one water molecule, and the residuals $\left(\nu_{\mathrm{obs}}-\nu_{\text {calc }}\right)$ for the fit reported in Table S6 (see Figure S2 for oxygen atom numbering).

\begin{tabular}{ccccccccc}
\hline$J^{\prime}$ & $K_{a}^{\prime}$ & $K_{c}^{\prime}$ & $\leftarrow$ & $J^{\prime \prime}$ & $K_{a}^{\prime \prime}$ & $K_{c}^{\prime \prime}$ & $\nu_{\mathrm{obs}}(\mathrm{MHz})$ & $\nu_{\mathrm{obs}}-\nu_{\text {calc }}$ \\
\hline & & & & & & & & \\
2 & 1 & 1 & $\leftarrow$ & 1 & 1 & 0 & 2634.4531 & 0.0081 \\
& & & & & & & & \\
3 & 1 & 3 & $\leftarrow$ & 2 & 1 & 2 & 3907.5646 & 0.0024 \\
3 & 0 & 3 & $\leftarrow$ & 2 & 0 & 2 & 3928.7268 & -0.0081 \\
3 & 2 & 2 & $\leftarrow$ & 2 & 2 & 1 & 3929.6574 & 0.0106 \\
3 & 2 & 1 & $\leftarrow$ & 2 & 2 & 0 & 3930.5890 & -0.0003 \\
3 & 1 & 2 & $\leftarrow$ & 2 & 1 & 1 & 3951.4900 & 0.0075 \\
& & & & & & & & \\
4 & 0 & 4 & $\leftarrow$ & 3 & 0 & 3 & 5237.1340 & 0.0013 \\
4 & 2 & 3 & $\leftarrow$ & 3 & 2 & 2 & 5239.2602 & -0.0036 \\
4 & 2 & 2 & $\leftarrow$ & 3 & 2 & 1 & 5241.6205 & 0.0015 \\
4 & 3 & 2 & $\leftarrow$ & 3 & 3 & 1 & 5239.8668 & -0.0013 \\
4 & 3 & 1 & $\leftarrow$ & 3 & 3 & 0 & 5239.8668 & -0.0013 \\
4 & 1 & 3 & $\leftarrow$ & 3 & 1 & 2 & 5268.3009 & 0.0064 \\
& & & & & & & & \\
5 & 1 & 5 & $\leftarrow$ & 4 & 1 & 4 & 6511.5611 & -0.0046 \\
5 & 0 & 5 & $\leftarrow$ & 4 & 0 & 4 & 6544.5280 & 0.0034 \\
5 & 2 & 4 & $\leftarrow$ & 4 & 2 & 3 & 6548.6579 & 0.0050 \\
5 & 2 & 3 & $\leftarrow$ & 4 & 2 & 2 & 6553.3556 & -0.0010 \\
5 & 3 & 3 & $\leftarrow$ & 4 & 3 & 2 & 6549.9041 & 0.0033 \\
5 & 3 & 2 & $\leftarrow$ & 4 & 3 & 1 & 6549.9562 & 0.0056 \\
5 & 1 & 4 & $\leftarrow$ & 4 & 1 & 3 & 6584.8238 & 0.0245 \\
& & & & & & & & \\
6 & 1 & 6 & $\leftarrow$ & 5 & 1 & 5 & 7813.0377 & -0.0004 \\
6 & 0 & 6 & $\leftarrow$ & 5 & 0 & 5 & 7850.6725 & -0.0023 \\
6 & 2 & 5 & $\leftarrow$ & 5 & 2 & 4 & 7857.7574 & 0.0007 \\
6 & 2 & 4 & $\leftarrow$ & 5 & 2 & 3 & 7865.9606 & -0.0017 \\
6 & 3 & 4 & $\leftarrow$ & 5 & 3 & 3 & 7859.9785 & 0.0005 \\
6 & 3 & 3 & $\leftarrow$ & 5 & 3 & 2 & 7860.1034 & -0.0077 \\
6 & 4 & 2 & $\leftarrow$ & 5 & 4 & 1 & 7859.4367 & -0.0041 \\
6 & 1 & 5 & $\leftarrow$ & 5 & 1 & 4 & 7900.9082 & -0.0020 \\
& & & & & & & & \\
7 & 1 & 7 & $\leftarrow$ & 6 & 1 & 6 & 9114.0735 & 0.0028 \\
7 & 0 & 7 & $\leftarrow$ & 6 & 0 & 6 & 9155.3714 & 0.0005 \\
7 & 1 & 6 & $\leftarrow$ & 6 & 1 & 5 & 9216.5262 & -0.0072 \\
& & & & & & & & \\
& & & & & & & $\mathrm{RMS}$ & $4.956 \mathrm{kHz}$ \\
\hline & & & & & & & &
\end{tabular}




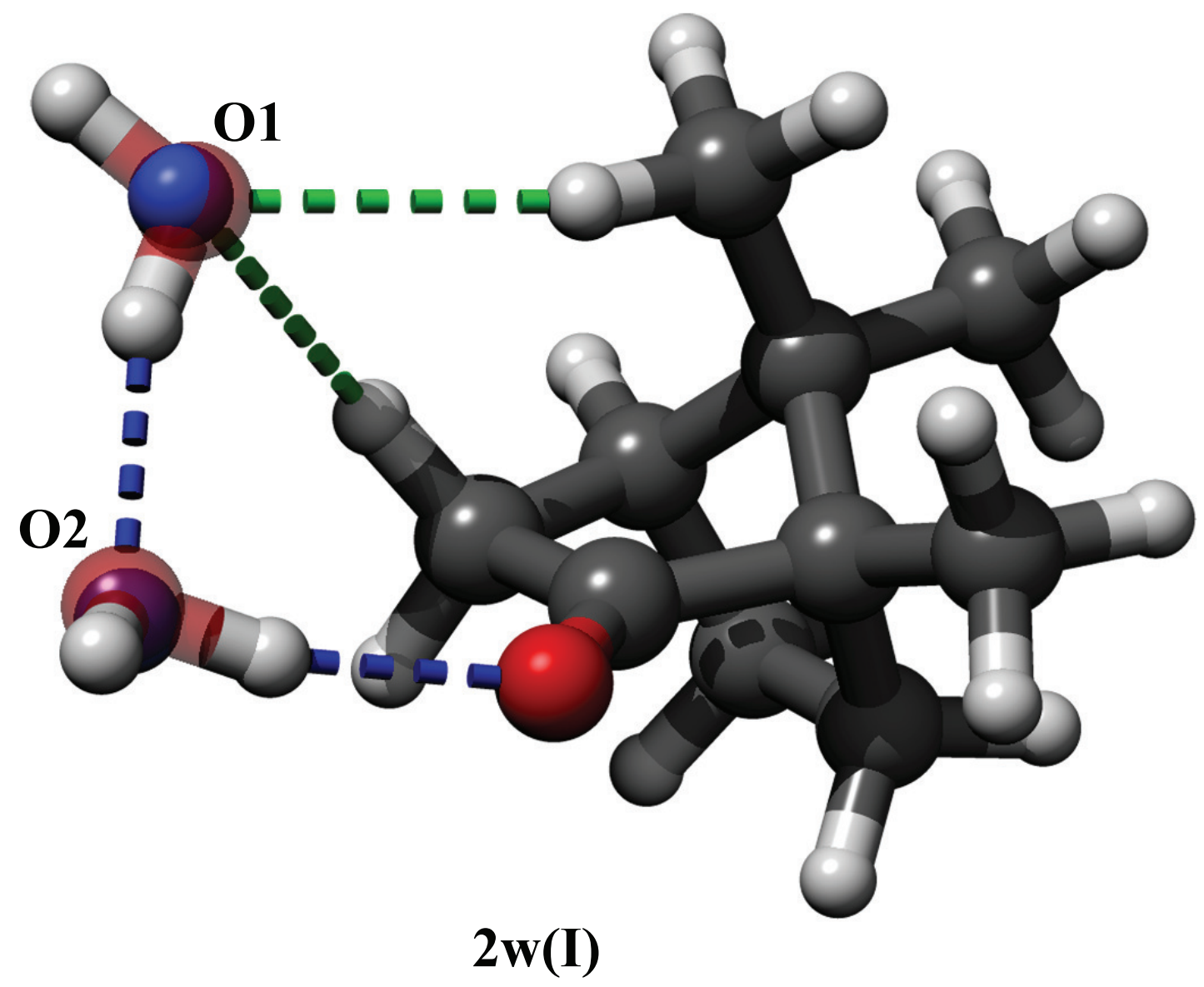

Figure S3: Calculated structures (MP2/6-311++G(d,p)) for the camphor complex $2 \mathrm{w}(\mathrm{I})$ with two water molecules. Oxygen atom numbering used for the $\mathrm{H}_{2}{ }^{18} \mathrm{O}$ isotopologues of the complex is shown. The blue/purple spheres represent the experimental oxygen positions from the Kraitchman analysis. Strong hydrogen bonds are depicted in blue, dispersive interactions are shown in green. 
Table S10: Experimentally determined rotational and quartic centrifugal distortion constants for the normal and the $\mathrm{H}_{2}{ }^{18} \mathrm{O}$ substituted species of the $2 \mathrm{w}(\mathrm{I})$ complex of camphor with two water molecules (see Figure S3 for oxygen atom numbering).

\begin{tabular}{lcccc}
\hline & normal & ${ }^{18} \mathrm{O} 1$ & ${ }^{18} \mathrm{O} 2$ & ${ }^{18} \mathrm{O} 1+{ }^{18} \mathrm{O} 2$ \\
\hline$A(\mathrm{MHz})$ & $1065.040(15)$ & $1050.842(10)$ & $1061.640(12)$ & $1046.710(14)$ \\
$B(\mathrm{MHz})$ & $514.70479(25)$ & $504.44445(18)$ & $499.50175(18)$ & $490.21446(22)$ \\
$C(\mathrm{MHz})$ & $472.43647(25)$ & $461.06391(17)$ & $459.010903(18)$ & $448.44807(22)$ \\
$\Delta_{J}(\mathrm{kHz})$ & $0.0526(16)$ & $0.0593(11)$ & $0.0575(11)$ & $0.0554(14)$ \\
$\Delta_{J K}(\mathrm{kHz})$ & $0.2025(76)$ & $0.2302(53)$ & $0.1982(54)$ & $0.2098(81)$ \\
$N_{\text {lines }}$ & 42 & 35 & 47 & 39 \\
$\sigma(\mathrm{kHz})$ & 3.85 & 2.51 & 2.92 & 3.33 \\
& & & & \\
$P_{a}\left(\mathrm{u} \AA^{2}\right)$ & $788.5469(30)$ & $808.5199(20)$ & $818.3640(25)$ & $837.5296(30)$ \\
$P_{b}\left(\mathrm{u} \AA^{2}\right)$ & $281.1820(30)$ & $287.5949(20)$ & $282.6339(25)$ & $289.4213(30)$ \\
$P_{c}\left(\mathrm{u} \AA^{2}\right)$ & $193.3343(30)$ & $193.3327(20)$ & $193.4022(25)$ & $193.4048(30)$ \\
\hline
\end{tabular}


Table S11: Experimental coordinates $(\AA)$ of the water oxygen atoms in the $2 \mathrm{w}(\mathrm{I})$ complex of camphor with two water molecules, compared with the results from ab initio calculations.

\begin{tabular}{rlrrrr}
\hline & \multicolumn{1}{c}{$r_{\mathrm{s}}{ }^{a}$} & \multicolumn{1}{c}{$r_{0}{ }^{b}$} & Calc $^{c}$ & Calc $^{d}$ & Calc $^{e}$ \\
\hline \multicolumn{5}{c}{$\mathrm{O} 1$} \\
$a$ & $3.8696(4)$ & $3.8716(4)$ & 3.9378 & 3.8593 & 3.8591 \\
$b$ & $0.8883(17)$ & $-0.8816(13)$ & -0.8252 & -0.8078 & -0.8563 \\
$c$ & $0.2364(64)$ & $0.1962(13)$ & 0.1522 & 0.2360 & 0.1921 \\
& & & & \\
& \multicolumn{5}{c}{$\mathrm{O} 2$} \\
$a$ & $3.1521(5)$ & $3.1532(4)$ & 3.0493 & 2.9677 & 3.1003 \\
$b$ & $1.8351(8)$ & $1.8328(7)$ & 1.8228 & 1.8055 & 1.8237 \\
$c$ & $0.1013(148)$ & $-0.0778(75)$ & -0.0631 & -0.0879 & -0.0919 \\
& & & & & \\
\hline
\end{tabular}

${ }^{a}$ Determined using Kraitchman equations, which result in only the absolute value, while uncertainties were estimated with the Costain criterion.

${ }^{b}$ Obtained from the least squares $r_{0}$ fit where the cited uncertainties only reflect those in the explicit parameters of the fit.

${ }^{c} \mathrm{MP} 2 / 6-311++\mathrm{G}(\mathrm{d}, \mathrm{p})$ calculation.

${ }^{d} \mathrm{M} 06-2 \mathrm{X} / 6-311++\mathrm{G}(\mathrm{d}, \mathrm{p})$ calculation.

${ }^{r}$ B3LYP-D3/aug-cc-pVTZ calculation. 
Table S12: Measured rotational transitions $\left(\nu_{\mathrm{obs}}\right)$ for the $2 \mathrm{w}(\mathrm{I})$ complex of camphor with two water molecules (main isotopologues), and the residuals $\left(\nu_{\mathrm{obs}}-\nu_{\text {calc }}\right)$ for the fit reported in Table 1.

\begin{tabular}{|c|c|c|c|c|c|c|c|c|}
\hline$J^{\prime}$ & $K_{a}^{\prime}$ & $K_{c}^{\prime}$ & $\leftarrow$ & $J^{\prime \prime}$ & $K_{a}^{\prime \prime}$ & $K_{c}^{\prime \prime}$ & $\nu_{\mathrm{obs}}(\mathrm{MHz})$ & $\nu_{\mathrm{obs}}-\nu_{\mathrm{calc}}$ \\
\hline 3 & 1 & 3 & $\leftarrow$ & 2 & 1 & 2 & 2896.5894 & 0.0004 \\
\hline 3 & 0 & 3 & $\leftarrow$ & 2 & 0 & 2 & 2952.0959 & -0.0002 \\
\hline 3 & 1 & 2 & $\leftarrow$ & 2 & 1 & 1 & 3023.3174 & 0.0047 \\
\hline 4 & 1 & 4 & $\leftarrow$ & 3 & 1 & 3 & 3859.5587 & -0.0042 \\
\hline 4 & 0 & 4 & $\leftarrow$ & 3 & 0 & 3 & 3925.5576 & 0.0082 \\
\hline 4 & 2 & 2 & $\leftarrow$ & 3 & 2 & 1 & 3969.7231 & 0.0012 \\
\hline 4 & 1 & 3 & $\leftarrow$ & 3 & 1 & 2 & 4028.1518 & 0.0022 \\
\hline 5 & 1 & 5 & $\leftarrow$ & 4 & 1 & 4 & 4820.5746 & -0.0028 \\
\hline 5 & 0 & 5 & $\leftarrow$ & 4 & 0 & 4 & 4890.8607 & -0.0005 \\
\hline 5 & 2 & 4 & $\leftarrow$ & 4 & 2 & 3 & 4930.4663 & -0.0017 \\
\hline 5 & 2 & 3 & $\leftarrow$ & 4 & 2 & 2 & 4975.2670 & -0.0012 \\
\hline 5 & 3 & 3 & $\leftarrow$ & 4 & 3 & 2 & 4942.8982 & 0.0054 \\
\hline 5 & 3 & 2 & $\leftarrow$ & 4 & 3 & 1 & 4944.5916 & 0.0026 \\
\hline 5 & 1 & 4 & $\leftarrow$ & 4 & 1 & 3 & 5030.2254 & 0.0027 \\
\hline 6 & 1 & 6 & $\leftarrow$ & 5 & 1 & 5 & 5779.4131 & 0.0024 \\
\hline 6 & 0 & 6 & $\leftarrow$ & 5 & 0 & 5 & 5847.7229 & -0.0028 \\
\hline 6 & 2 & 5 & $\leftarrow$ & 5 & 2 & 4 & 5912.2712 & -0.0031 \\
\hline 6 & 2 & 4 & $\leftarrow$ & 5 & 2 & 3 & 5987.2679 & 0.0010 \\
\hline 6 & 3 & 4 & $\leftarrow$ & 5 & 3 & 3 & 5933.4977 & -0.0026 \\
\hline 6 & 3 & 3 & $\leftarrow$ & 5 & 3 & 2 & 5937.9854 & -0.0036 \\
\hline 6 & 5 & 2 & $\leftarrow$ & 5 & 5 & 1 & 5928.6103 & 0.0047 \\
\hline 6 & 5 & 1 & $\leftarrow$ & 5 & 5 & 0 & 5928.6103 & 0.0041 \\
\hline 6 & 1 & 5 & $\leftarrow$ & 5 & 1 & 4 & 6028.5289 & -0.0022 \\
\hline 7 & 1 & 7 & $\leftarrow$ & 6 & 1 & 6 & 6735.9919 & 0.0061 \\
\hline 7 & 0 & 7 & $\leftarrow$ & 6 & 0 & 6 & 6797.2178 & -0.0078 \\
\hline 7 & 2 & 6 & $\leftarrow$ & 6 & 2 & 5 & 6891.7765 & -0.0064 \\
\hline 7 & 2 & 5 & $\leftarrow$ & 6 & 2 & 4 & 7004.1945 & -0.0009 \\
\hline 7 & 3 & 5 & $\leftarrow$ & 6 & 3 & 4 & 6924.5842 & -0.0072 \\
\hline 7 & 3 & 4 & $\leftarrow$ & 6 & 3 & 3 & 6934.5591 & -0.0022 \\
\hline 7 & 4 & 4 & $\leftarrow$ & 6 & 4 & 3 & 6921.4240 & -0.0018 \\
\hline 7 & 4 & 3 & $\leftarrow$ & 6 & 4 & 2 & 6921.7040 & -0.0004 \\
\hline 7 & 5 & 3 & $\leftarrow$ & 6 & 5 & 2 & 6918.1747 & 0.0045 \\
\hline 7 & 5 & 2 & $\leftarrow$ & 6 & 5 & 1 & 6918.1747 & 0.0011 \\
\hline 7 & 1 & 6 & $\leftarrow$ & 6 & 1 & 5 & 7021.8897 & -0.0009 \\
\hline 8 & 1 & 8 & $\leftarrow$ & 7 & 1 & 7 & 7690.3674 & 0.0056 \\
\hline 8 & 0 & 8 & $\leftarrow$ & 7 & 0 & 7 & 7741.5027 & 0.0040 \\
\hline 8 & 2 & 7 & $\leftarrow$ & 7 & 2 & 6 & 7868.6612 & -0.0067 \\
\hline 8 & 2 & 6 & $\leftarrow$ & 7 & 2 & 5 & 8023.4320 & 0.0018 \\
\hline 8 & 3 & 6 & $\leftarrow$ & 7 & 3 & 5 & 7915.8108 & -0.0003 \\
\hline 8 & 3 & 5 & $\leftarrow$ & 7 & 3 & 4 & 7935.3506 & 0.0024 \\
\hline 8 & 4 & 5 & $\leftarrow$ & 7 & 4 & 4 & 7913.1168 & 0.0032 \\
\hline 8 & 4 & 4 & $\leftarrow$ & 7 & 4 & 3 & 7913.8739 & -0.0014 \\
\hline 8 & 5 & 4 & $\leftarrow$ & 7 & 5 & 3 & 7908.4148 & 0.0046 \\
\hline 8 & 5 & 3 & $\leftarrow$ & 7 & 5 & 2 & 7908.4148 & -0.0087 \\
\hline \multirow[t]{2}{*}{8} & 1 & 7 & $\leftarrow$ & 7 & 1 & 6 & 8008.9647 & 0.0066 \\
\hline & & & & & & & RMS & $3.848 \mathrm{kHz}$ \\
\hline
\end{tabular}


Table S13: Measured rotational transitions $\left(\nu_{\mathrm{obs}}\right)$ for the $2 \mathrm{w}(\mathrm{I})-{ }^{18} \mathrm{O} 1$ complex of camphor with two water molecules, and the residuals $\left(\nu_{\text {obs }}-\nu_{\text {calc }}\right)$ for the fit reported in Table S10 (see Figure S3 for oxygen atom numbering).

\begin{tabular}{|c|c|c|c|c|c|c|c|c|}
\hline$J^{\prime}$ & $K_{a}^{\prime}$ & $K_{c}^{\prime}$ & $\leftarrow$ & $J^{\prime \prime}$ & $K_{a}^{\prime \prime}$ & $K_{c}^{\prime \prime}$ & $\nu_{\mathrm{obs}}(\mathrm{MHz})$ & $\nu_{\text {obs }}-\nu_{\text {calc }}$ \\
\hline 3 & 1 & 3 & $\leftarrow$ & 2 & 1 & 2 & 2829.9318 & -0.0055 \\
\hline 3 & 0 & 3 & $\leftarrow$ & 2 & 0 & 2 & 2886.6437 & -0.0014 \\
\hline 4 & 1 & 4 & $\leftarrow$ & 3 & 1 & 3 & 3770.5462 & -0.0016 \\
\hline 4 & 0 & 4 & $\leftarrow$ & 3 & 0 & 3 & 3837.6738 & -0.0015 \\
\hline 4 & 2 & 3 & $\leftarrow$ & 3 & 2 & 2 & 3860.0792 & 0.0001 \\
\hline 4 & 2 & 2 & $\leftarrow$ & 3 & 2 & 1 & 3884.4240 & 0.0047 \\
\hline 4 & 1 & 3 & $\leftarrow$ & 3 & 1 & 2 & 3943.5382 & 0.0006 \\
\hline 5 & 1 & 5 & $\leftarrow$ & 4 & 1 & 4 & 4709.0928 & -0.0013 \\
\hline 5 & 2 & 4 & $\leftarrow$ & 4 & 2 & 3 & 4821.9892 & -0.0001 \\
\hline 5 & 2 & 3 & $\leftarrow$ & 4 & 2 & 2 & 4869.3255 & 0.0018 \\
\hline 5 & 3 & 2 & $\leftarrow$ & 4 & 3 & 1 & 4836.9820 & 0.0014 \\
\hline 6 & 1 & 6 & $\leftarrow$ & 5 & 1 & 5 & 5645.3521 & -0.0003 \\
\hline 6 & 0 & 6 & $\leftarrow$ & 5 & 0 & 5 & 5713.8682 & -0.0005 \\
\hline 6 & 2 & 5 & $\leftarrow$ & 5 & 2 & 4 & 5781.8464 & 0.0010 \\
\hline 6 & 2 & 4 & $\leftarrow$ & 5 & 2 & 3 & 5860.8820 & 0.0011 \\
\hline 6 & 3 & 4 & $\leftarrow$ & 5 & 3 & 3 & 5804.2596 & -0.0027 \\
\hline 6 & 3 & 3 & $\leftarrow$ & 5 & 3 & 2 & 5809.1671 & -0.0010 \\
\hline 6 & 5 & 2 & $\leftarrow$ & 5 & 5 & 1 & 5799.1459 & 0.0023 \\
\hline 6 & 5 & 1 & $\leftarrow$ & 5 & 5 & 0 & 5799.1459 & 0.0015 \\
\hline 6 & 1 & 5 & $\leftarrow$ & 5 & 1 & 4 & 5900.7301 & 0.0008 \\
\hline 7 & 1 & 7 & $\leftarrow$ & 6 & 1 & 6 & 6579.2542 & -0.0001 \\
\hline 7 & 2 & 6 & $\leftarrow$ & 6 & 2 & 5 & 6739.2656 & -0.0042 \\
\hline 7 & 2 & 5 & $\leftarrow$ & 6 & 2 & 4 & 6857.3592 & 0.0057 \\
\hline 7 & 3 & 4 & $\leftarrow$ & 6 & 3 & 3 & 6784.7590 & -0.0014 \\
\hline 7 & 5 & 3 & $\leftarrow$ & 6 & 5 & 2 & 6767.2203 & 0.0032 \\
\hline 7 & 5 & 2 & $\leftarrow$ & 6 & 5 & 1 & 6767.2203 & -0.0006 \\
\hline 7 & 1 & 6 & $\leftarrow$ & 6 & 1 & 5 & 6872.0293 & 0.0015 \\
\hline 8 & 1 & 8 & $\leftarrow$ & 7 & 1 & 7 & 7510.8815 & 0.0052 \\
\hline 8 & 0 & 8 & $\leftarrow$ & 7 & 0 & 7 & 7561.1221 & 0.0021 \\
\hline 8 & 2 & 7 & $\leftarrow$ & 7 & 2 & 6 & 7693.9210 & -0.0009 \\
\hline 8 & 2 & 6 & $\leftarrow$ & 7 & 2 & 5 & 7855.8837 & -0.0039 \\
\hline 8 & 3 & 6 & $\leftarrow$ & 7 & 3 & 5 & 7743.5658 & -0.0047 \\
\hline 8 & 3 & 5 & $\leftarrow$ & 7 & 3 & 4 & 7764.8745 & 0.0011 \\
\hline 8 & 4 & 5 & $\leftarrow$ & 7 & 4 & 4 & 7740.9696 & 0.0002 \\
\hline 8 & 4 & 4 & $\leftarrow$ & 7 & 4 & 3 & 7741.8264 & -0.0025 \\
\hline 8 & 5 & 4 & $\leftarrow$ & 7 & 5 & 3 & 7736.0140 & 0.0085 \\
\hline 8 & 5 & 3 & $\leftarrow$ & 7 & 5 & 2 & 7736.0140 & -0.0070 \\
\hline \multirow[t]{2}{*}{8} & 1 & 7 & $\leftarrow$ & 7 & 1 & 6 & 7836.6025 & 0.0001 \\
\hline & & & & & & & RMS & $2.509 \mathrm{kHz}$ \\
\hline
\end{tabular}


Table S14: Measured rotational transitions $\left(\nu_{\mathrm{obs}}\right)$ for the $2 \mathrm{w}(\mathrm{I})-{ }^{18} \mathrm{O} 2$ complex of camphor with two water molecules, and the residuals $\left(\nu_{\text {obs }}-\nu_{\text {calc }}\right)$ for the fit reported in Table S10 (see Figure S3 for oxygen atom numbering).

\begin{tabular}{|c|c|c|c|c|c|c|c|c|}
\hline$J^{\prime}$ & $K_{a}^{\prime}$ & $K_{c}^{\prime}$ & $\leftarrow$ & $J^{\prime \prime}$ & $K_{a}^{\prime \prime}$ & $K_{c}^{\prime \prime}$ & $\nu_{\mathrm{obs}}(\mathrm{MHz})$ & $\nu_{\mathrm{obs}}-\nu_{\mathrm{calc}}$ \\
\hline 3 & 1 & 3 & $\leftarrow$ & 2 & 1 & 2 & 2813.5447 & -0.0009 \\
\hline 3 & 0 & 3 & $\leftarrow$ & 2 & 0 & 2 & 2867.1586 & -0.0007 \\
\hline 3 & 2 & 2 & $\leftarrow$ & 2 & 2 & 1 & 2875.5549 & 0.0034 \\
\hline 3 & 2 & 1 & $\leftarrow$ & 2 & 2 & 0 & 2883.9453 & -0.0028 \\
\hline 3 & 1 & 2 & $\leftarrow$ & 2 & 1 & 1 & 2934.9274 & 0.0023 \\
\hline 4 & 1 & 4 & $\leftarrow$ & 3 & 1 & 3 & 3749.0798 & -0.0031 \\
\hline 4 & 0 & 4 & $\leftarrow$ & 3 & 0 & 3 & 3813.3191 & 0.0007 \\
\hline 4 & 2 & 3 & $\leftarrow$ & 3 & 2 & 2 & 3832.4198 & -0.0011 \\
\hline 4 & 2 & 2 & $\leftarrow$ & 3 & 2 & 1 & 3853.1734 & 0.0042 \\
\hline 4 & 1 & 3 & $\leftarrow$ & 3 & 1 & 2 & 3910.6063 & 0.0043 \\
\hline 5 & 1 & 5 & $\leftarrow$ & 4 & 1 & 4 & 4682.8348 & -0.0002 \\
\hline 5 & 0 & 5 & $\leftarrow$ & 4 & 0 & 4 & 4752.0278 & -0.0009 \\
\hline 5 & 2 & 4 & $\leftarrow$ & 4 & 2 & 3 & 4787.8798 & -0.0026 \\
\hline 5 & 2 & 3 & $\leftarrow$ & 4 & 2 & 2 & 4828.4173 & 0.0033 \\
\hline 5 & 3 & 3 & $\leftarrow$ & 4 & 3 & 2 & 4799.1093 & 0.0000 \\
\hline 5 & 4 & 1 & $\leftarrow$ & 4 & 4 & 0 & 4797.2341 & -0.0090 \\
\hline 5 & 1 & 4 & $\leftarrow$ & 4 & 1 & 3 & 4883.8151 & 0.0025 \\
\hline 6 & 1 & 6 & $\leftarrow$ & 5 & 1 & 5 & 5614.5839 & -0.0014 \\
\hline 6 & 0 & 6 & $\leftarrow$ & 5 & 0 & 5 & 5682.8509 & 0.0002 \\
\hline 6 & 2 & 5 & $\leftarrow$ & 5 & 2 & 4 & 5741.5909 & -0.0036 \\
\hline 6 & 2 & 4 & $\leftarrow$ & 5 & 2 & 3 & 5809.7525 & 0.0002 \\
\hline 6 & 3 & 4 & $\leftarrow$ & 5 & 3 & 3 & 5760.8121 & -0.0025 \\
\hline 6 & 3 & 3 & $\leftarrow$ & 5 & 3 & 2 & 5764.6178 & -0.0004 \\
\hline 6 & 4 & 3 & $\leftarrow$ & 5 & 4 & 2 & 5758.1909 & 0.0054 \\
\hline 6 & 4 & 2 & $\leftarrow$ & 5 & 4 & 1 & 5758.2525 & 0.0002 \\
\hline 6 & 5 & 2 & $\leftarrow$ & 5 & 5 & 1 & 5756.2993 & 0.0055 \\
\hline 6 & 5 & 1 & $\leftarrow$ & 5 & 5 & 0 & 5756.2993 & 0.0051 \\
\hline 6 & 1 & 5 & $\leftarrow$ & 5 & 1 & 4 & 5853.6768 & -0.0008 \\
\hline 7 & 1 & 7 & $\leftarrow$ & 6 & 1 & 6 & 6544.2420 & -0.0002 \\
\hline 7 & 0 & 7 & $\leftarrow$ & 6 & 0 & 6 & 6606.5551 & -0.0005 \\
\hline 7 & 2 & 6 & $\leftarrow$ & 6 & 2 & 5 & 6693.2271 & -0.0042 \\
\hline 7 & 2 & 5 & $\leftarrow$ & 6 & 2 & 4 & 6796.0213 & 0.0006 \\
\hline 7 & 3 & 5 & $\leftarrow$ & 6 & 3 & 4 & 6723.0147 & 0.0018 \\
\hline 7 & 3 & 4 & $\leftarrow$ & 6 & 3 & 3 & 6731.4746 & 0.0005 \\
\hline 7 & 4 & 4 & $\leftarrow$ & 6 & 4 & 3 & 6719.9351 & 0.0004 \\
\hline 7 & 4 & 3 & $\leftarrow$ & 6 & 4 & 2 & 6720.1570 & 0.0006 \\
\hline 7 & 5 & 3 & $\leftarrow$ & 6 & 5 & 2 & 6716.9887 & -0.0009 \\
\hline 7 & 5 & 2 & $\leftarrow$ & 6 & 5 & 1 & 6716.9887 & -0.0034 \\
\hline 7 & 1 & 6 & $\leftarrow$ & 6 & 1 & 5 & 6819.1585 & 0.0008 \\
\hline 8 & 1 & 8 & $\leftarrow$ & 7 & 1 & 7 & 7471.8395 & 0.0033 \\
\hline 8 & 0 & 8 & $\leftarrow$ & 7 & 0 & 7 & 7524.9420 & 0.0062 \\
\hline 8 & 2 & 7 & $\leftarrow$ & 7 & 2 & 6 & 7642.4874 & -0.0051 \\
\hline 8 & 2 & 6 & $\leftarrow$ & 7 & 2 & 5 & 7784.9989 & 0.0001 \\
\hline 8 & 3 & 5 & $\leftarrow$ & 7 & 3 & 4 & 7702.0280 & -0.0013 \\
\hline 8 & 3 & 6 & $\leftarrow$ & 7 & 3 & 5 & 7685.4077 & 0.0006 \\
\hline 8 & 4 & 5 & $\leftarrow$ & 7 & 4 & 4 & 7682.5654 & 0.0038 \\
\hline 8 & 4 & 4 & $\leftarrow$ & 7 & 4 & 3 & 7683.1671 & -0.0010 \\
\hline 8 & 5 & 4 & $\leftarrow$ & 7 & 5 & 3 & 7678.2950 & 0.0029 \\
\hline 8 & 5 & 3 & $\leftarrow$ & 7 & 5 & 2 & 7678.2950 & -0.0070 \\
\hline \multirow[t]{2}{*}{8} & 1 & 7 & $\leftarrow$ & 7 & 1 & 6 & 7779.0625 & -0.0031 \\
\hline & & & & & & & RMS & $2.924 \mathrm{kHz}$ \\
\hline
\end{tabular}


Table S15: Measured rotational transitions $\left(\nu_{\mathrm{obs}}\right)$ for the $2 \mathrm{w}(\mathrm{I})-{ }^{18} \mathrm{O} 1^{18} \mathrm{O} 2$ complex of camphor with two water molecules, and the residuals $\left(\nu_{\text {obs }}-\nu_{\text {calc }}\right)$ for the fit reported in Table S10 (see Figure S3 for oxygen atom numbering).

\begin{tabular}{|c|c|c|c|c|c|c|c|c|}
\hline$J^{\prime}$ & $K_{a}^{\prime}$ & $K_{c}^{\prime}$ & $\leftarrow$ & $J^{\prime \prime}$ & $K_{a}^{\prime \prime}$ & $K_{c}^{\prime \prime}$ & $\nu_{\mathrm{obs}}(\mathrm{MHz})$ & $\nu_{\mathrm{obs}}-\nu_{\mathrm{calc}}$ \\
\hline 3 & 1 & 3 & $\leftarrow$ & 2 & 1 & 2 & 2751.9489 & -0.0034 \\
\hline 3 & 0 & 3 & $\leftarrow$ & 2 & 0 & 2 & 2806.9696 & -0.0009 \\
\hline 3 & 1 & 2 & $\leftarrow$ & 2 & 1 & 1 & 2877.1780 & 0.0033 \\
\hline 4 & 1 & 4 & $\leftarrow$ & 3 & 1 & 3 & 3666.7939 & -0.0018 \\
\hline 4 & 0 & 4 & $\leftarrow$ & 3 & 0 & 3 & 3732.3880 & -0.0004 \\
\hline 4 & 2 & 3 & $\leftarrow$ & 3 & 2 & 2 & 3752.8681 & 0.0009 \\
\hline 4 & 2 & 2 & $\leftarrow$ & 3 & 2 & 1 & 3775.1153 & 0.0027 \\
\hline 4 & 1 & 3 & $\leftarrow$ & 3 & 1 & 2 & 3833.4035 & 0.0022 \\
\hline 5 & 1 & 5 & $\leftarrow$ & 4 & 1 & 4 & 4579.7344 & -0.0024 \\
\hline 5 & 2 & 4 & $\leftarrow$ & 4 & 2 & 3 & 4688.2420 & -0.0052 \\
\hline 5 & 2 & 3 & $\leftarrow$ & 4 & 2 & 2 & 4731.6280 & 0.0038 \\
\hline 5 & 3 & 3 & $\leftarrow$ & 4 & 3 & 2 & 4700.2739 & 0.0028 \\
\hline 5 & 3 & 2 & $\leftarrow$ & 4 & 3 & 1 & 4701.8718 & -0.0029 \\
\hline 5 & 4 & 2 & $\leftarrow$ & 4 & 4 & 1 & 4698.2870 & 0.0062 \\
\hline 5 & 4 & 1 & $\leftarrow$ & 4 & 4 & 0 & 4698.2870 & -0.0109 \\
\hline 5 & 1 & 4 & $\leftarrow$ & 4 & 1 & 3 & 4786.9675 & 0.0024 \\
\hline 6 & 1 & 6 & $\leftarrow$ & 5 & 1 & 5 & 5490.5557 & 0.0006 \\
\hline 6 & 0 & 6 & $\leftarrow$ & 5 & 0 & 5 & 5559.1184 & 0.0012 \\
\hline 6 & 2 & 5 & $\leftarrow$ & 5 & 2 & 4 & 5621.7522 & 0.0009 \\
\hline 6 & 2 & 4 & $\leftarrow$ & 5 & 2 & 3 & 5694.4885 & 0.0046 \\
\hline 6 & 3 & 4 & $\leftarrow$ & 5 & 3 & 3 & 5642.3122 & 0.0039 \\
\hline 6 & 3 & 3 & $\leftarrow$ & 5 & 3 & 2 & 5646.5552 & 0.0017 \\
\hline 6 & 1 & 5 & $\leftarrow$ & 5 & 1 & 4 & 5736.9060 & -0.0016 \\
\hline 7 & 1 & 7 & $\leftarrow$ & 6 & 1 & 6 & 6399.1681 & 0.0009 \\
\hline 7 & 0 & 7 & $\leftarrow$ & 6 & 0 & 6 & 6461.0360 & -0.0002 \\
\hline 7 & 2 & 6 & $\leftarrow$ & 6 & 2 & 5 & 6553.0259 & -0.0058 \\
\hline 7 & 2 & 5 & $\leftarrow$ & 6 & 2 & 4 & 6662.2970 & -0.0025 \\
\hline 7 & 3 & 4 & $\leftarrow$ & 6 & 3 & 3 & 6594.2705 & 0.0009 \\
\hline 7 & 4 & 4 & $\leftarrow$ & 6 & 4 & 3 & 6581.6818 & -0.0040 \\
\hline 7 & 4 & 3 & $\leftarrow$ & 6 & 4 & 2 & 6581.9378 & -0.0056 \\
\hline 7 & 1 & 6 & $\leftarrow$ & 6 & 1 & 5 & 6682.0946 & 0.0001 \\
\hline 8 & 1 & 8 & $\leftarrow$ & 7 & 1 & 7 & 7305.6245 & 0.0027 \\
\hline 8 & 0 & 8 & $\leftarrow$ & 7 & 0 & 7 & 7357.6704 & 0.0038 \\
\hline 8 & 2 & 7 & $\leftarrow$ & 7 & 2 & 6 & 7481.7668 & -0.0042 \\
\hline 8 & 2 & 6 & $\leftarrow$ & 7 & 2 & 5 & 7632.5880 & 0.0012 \\
\hline 8 & 3 & 5 & $\leftarrow$ & 7 & 3 & 4 & 7546.0156 & -0.0067 \\
\hline 8 & 4 & 5 & $\leftarrow$ & 7 & 4 & 4 & 7524.7564 & 0.0093 \\
\hline 8 & 4 & 4 & $\leftarrow$ & 7 & 4 & 3 & 7525.4527 & 0.0012 \\
\hline 8 & 5 & 4 & $\leftarrow$ & 7 & 5 & 3 & 7520.1930 & 0.0069 \\
\hline 8 & 5 & 3 & $\leftarrow$ & 7 & 5 & 2 & 7520.1930 & -0.0051 \\
\hline \multirow[t]{2}{*}{8} & 1 & 7 & $\leftarrow$ & 7 & 1 & 6 & 7621.2339 & -0.0017 \\
\hline & & & & & & & RMS & $3.330 \mathrm{kHz}$ \\
\hline
\end{tabular}




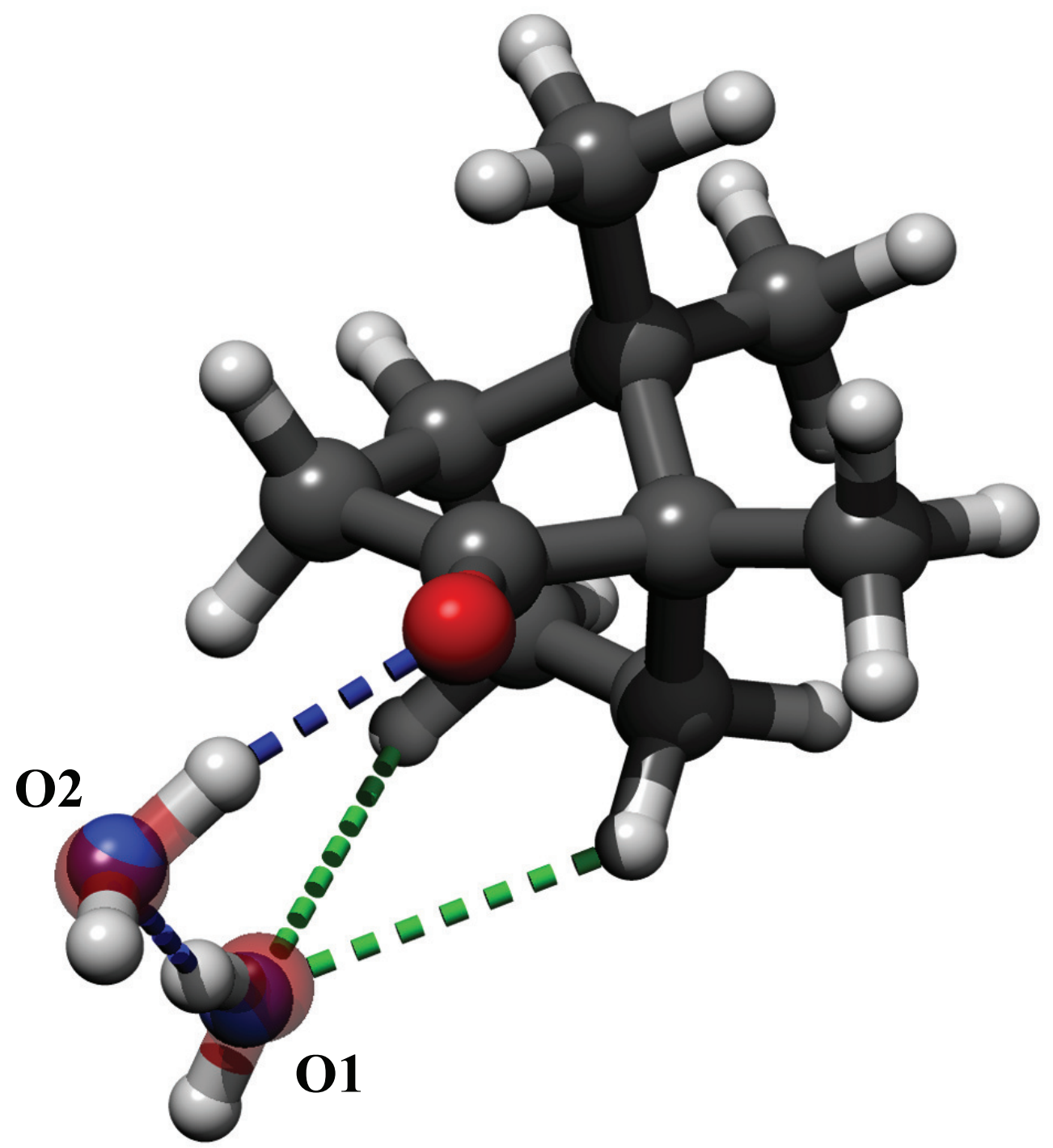

$2 \mathrm{w}(\mathrm{II})$

Figure S4: Calculated structures $(\mathrm{MP} 2 / 6-311++\mathrm{G}(\mathrm{d}, \mathrm{p})$ ) for the camphor complex $2 \mathrm{w}$ (II) with two water molecules. Oxygen atom numbering used for the $\mathrm{H}_{2}{ }^{18} \mathrm{O}$ isotopologues of the complex is shown. The blue/purple spheres represent the experimental oxygen positions from the Kraitchman analysis. Strong hydrogen bonds are depicted in blue, dispersive interactions are shown in green. 
Table S16: Experimentally determined rotational and quartic centrifugal distortion constants for the normal and the $\mathrm{H}_{2}{ }^{18} \mathrm{O}$ substituted species of the $2 \mathrm{w}$ (II) complex of camphor with two water molecules (see Figure S4 for oxygen atom numbering).

\begin{tabular}{lcccc}
\hline & normal & ${ }^{18} \mathrm{O} 1$ & ${ }^{18} \mathrm{O} 2$ & ${ }^{18} \mathrm{O} 1+{ }^{18} \mathrm{O} 2$ \\
\hline$A(\mathrm{MHz})$ & $1134.2098(40)$ & $1120.348(10)$ & $1129.058(11)$ & $1114.403(11)$ \\
$B(\mathrm{MHz})$ & $528.38221(28)$ & $518.40518(18)$ & $512.61445(17)$ & $503.59665(19)$ \\
$C(\mathrm{MHz})$ & $477.44192(25)$ & $467.11246(18)$ & $464.00901(18)$ & $454.41412(19)$ \\
$\Delta_{J}(\mathrm{kHz})$ & $0.0629(21)$ & $0.0652(12)$ & $0.0643(11)$ & $0.0613(12)$ \\
$\Delta_{J K}(\mathrm{kHz}$ & $0.169(16)$ & $0.2229(73)$ & $0.2088(54)$ & $0.2237(72)$ \\
$N_{\text {lines }}$ & 39 & 41 & 44 & 42 \\
$\sigma(\mathrm{kHz})$ & 4.46 & 2.86 & 2.80 & 2.93 \\
& & & & \\
$P_{a}\left(\mathrm{u} \AA^{2}\right)$ & $784.7004(9)$ & $802.8514(20)$ & $813.7158(20)$ & $831.0984(20)$ \\
$P_{b}\left(\mathrm{u} \AA^{2}\right)$ & $273.8136(9)$ & $279.0699(20)$ & $275.4418(20)$ & $281.0567(20)$ \\
$P_{c}\left(\mathrm{u} \AA^{2}\right)$ & $171.7644(9)$ & $172.0211(20)$ & $172.1693(20)$ & $172.4408(20)$ \\
\hline
\end{tabular}


Table S17: Experimental coordinates $(\AA)$ of the water oxygen atoms in the camphor complex $(2 \mathrm{w}(\mathrm{II}))$ with two water molecules, compared with the results from ab initio calculations.

\begin{tabular}{llrrrr}
\hline & \multicolumn{1}{c}{$r_{\mathrm{s}}{ }^{a}$} & \multicolumn{1}{c}{$r_{0}{ }^{b}$} & Calc $^{c}$ & Calc $^{d}$ & Calc $^{e}$ \\
\hline \multicolumn{5}{c}{$\mathrm{O} 1$} \\
$a$ & $3.0091(5)$ & $3.0090(2)$ & 2.8820 & 2.7616 & 2.9876 \\
$b$ & $1.6547(9)$ & $1.6554(4)$ & 1.6365 & 1.5917 & 1.6381 \\
$c$ & $0.3743(40)$ & $0.3749(4)$ & 0.3935 & 0.4545 & 0.3853 \\
& & & & & \\
& \multicolumn{5}{c}{$\mathrm{O} 2$} \\
$a$ & $3.8183(4)$ & $3.8176(2)$ & 3.8748 & 3.7089 & 3.7809 \\
$b$ & $0.9274(16)$ & $-0.9300(6)$ & -0.8665 & -0.8281 & -0.9209 \\
$c$ & $0.4605(33)$ & $-0.4690(8)$ & -0.4419 & -0.5638 & -0.4729 \\
& & & & & \\
\hline
\end{tabular}

${ }^{a}$ Determined using Kraitchman equations, which result in only the absolute value, while uncertainties were estimated with the Costain criterion.

${ }^{b}$ Obtained from the least squares $r_{0}$ fit where the cited uncertainties only reflect those in the explicit parameters of the fit.

${ }^{c} \mathrm{MP} 2 / 6-311++\mathrm{G}(\mathrm{d}, \mathrm{p})$ calculation.

${ }^{d} \mathrm{M} 06-2 \mathrm{X} / 6-311++\mathrm{G}(\mathrm{d}, \mathrm{p})$ calculation.

${ }^{r}$ B3LYP-D3/aug-cc-pVTZ calculation. 
Table S18: Measured rotational transitions $\left(\nu_{\mathrm{obs}}\right)$ for the $2 \mathrm{w}(\mathrm{II})$ complex of camphor with two water molecules (main isotopologues), and the residuals $\left(\nu_{\mathrm{obs}}-\nu_{\text {calc }}\right)$ for the fit reported in Table 1.

\begin{tabular}{|c|c|c|c|c|c|c|c|c|}
\hline$J^{\prime}$ & $K_{a}^{\prime}$ & $K_{c}^{\prime}$ & $\leftarrow$ & $J^{\prime \prime}$ & $K_{a}^{\prime \prime}$ & $K_{c}^{\prime \prime}$ & $\nu_{\mathrm{obs}}(\mathrm{MHz})$ & $\nu_{\mathrm{obs}}-\nu_{\mathrm{calc}}$ \\
\hline \multicolumn{9}{|c|}{$a$-type transitions: } \\
\hline 3 & 1 & 3 & $\leftarrow$ & 2 & 1 & 2 & 2939.1849 & 0.0002 \\
\hline 3 & 0 & 3 & $\leftarrow$ & 2 & 0 & 2 & 3005.2230 & -0.0004 \\
\hline 3 & 1 & 2 & $\leftarrow$ & 2 & 1 & 1 & 3091.8926 & 0.0036 \\
\hline 4 & 1 & 4 & $\leftarrow$ & 3 & 1 & 3 & 3915.5821 & 0.0068 \\
\hline 4 & 0 & 4 & $\leftarrow$ & 3 & 0 & 3 & 3993.1457 & 0.0007 \\
\hline 4 & 2 & 3 & $\leftarrow$ & 3 & 2 & 2 & 4020.8856 & 0.0076 \\
\hline 4 & 2 & 2 & $\leftarrow$ & 3 & 2 & 1 & 4051.0071 & -0.0026 \\
\hline 4 & 1 & 3 & $\leftarrow$ & 3 & 1 & 2 & 4118.6377 & -0.0008 \\
\hline 5 & 1 & 5 & $\leftarrow$ & 4 & 1 & 4 & 4889.4349 & 0.0009 \\
\hline 5 & 0 & 5 & $\leftarrow$ & 4 & 0 & 4 & 4970.6367 & -0.0022 \\
\hline 5 & 2 & 4 & $\leftarrow$ & 4 & 2 & 3 & 5022.2337 & -0.0083 \\
\hline 5 & 2 & 3 & $\leftarrow$ & 4 & 2 & 2 & 5080.6583 & -0.0048 \\
\hline 5 & 3 & 3 & $\leftarrow$ & 4 & 3 & 2 & 5038.4896 & 0.0042 \\
\hline 5 & 3 & 2 & $\leftarrow$ & 4 & 3 & 1 & 5040.9178 & 0.0023 \\
\hline 5 & 1 & 4 & $\leftarrow$ & 4 & 1 & 3 & 5141.7041 & -0.0011 \\
\hline 6 & 1 & 6 & $\leftarrow$ & 5 & 1 & 5 & 5860.5079 & 0.0006 \\
\hline 6 & 0 & 6 & $\leftarrow$ & 5 & 0 & 5 & 5937.6632 & 0.0002 \\
\hline 6 & 2 & 5 & $\leftarrow$ & 5 & 2 & 4 & 6021.0570 & -0.0048 \\
\hline 6 & 2 & 4 & $\leftarrow$ & 5 & 2 & 3 & 6118.1540 & -0.0041 \\
\hline 6 & 3 & 4 & $\leftarrow$ & 5 & 3 & 3 & 6048.7199 & -0.0020 \\
\hline 6 & 3 & 3 & $\leftarrow$ & 5 & 3 & 2 & 6055.1371 & -0.0057 \\
\hline 6 & 1 & 5 & $\leftarrow$ & 5 & 1 & 4 & 6159.7266 & -0.0005 \\
\hline 7 & 1 & 7 & $\leftarrow$ & 6 & 1 & 6 & 6828.7493 & 0.0101 \\
\hline 7 & 0 & 7 & $\leftarrow$ & 6 & 0 & 6 & 6896.0378 & -0.0043 \\
\hline 7 & 2 & 6 & $\leftarrow$ & 6 & 2 & 5 & 7016.8745 & -0.0002 \\
\hline 7 & 2 & 5 & $\leftarrow$ & 6 & 2 & 4 & 7161.0869 & 0.0070 \\
\hline 7 & 3 & 5 & $\leftarrow$ & 6 & 3 & 4 & 7059.4741 & 0.0058 \\
\hline 7 & 3 & 4 & $\leftarrow$ & 6 & 3 & 3 & 7073.6978 & 0.0052 \\
\hline 7 & 4 & 4 & $\leftarrow$ & 6 & 4 & 3 & 7055.8058 & -0.0012 \\
\hline 7 & 4 & 3 & $\leftarrow$ & 6 & 4 & 2 & 7056.2382 & -0.0041 \\
\hline 7 & 1 & 6 & $\leftarrow$ & 6 & 1 & 5 & 7171.0839 & -0.0071 \\
\hline 8 & 1 & 8 & $\leftarrow$ & 7 & 1 & 7 & 7794.2504 & -0.0040 \\
\hline 8 & 2 & 7 & $\leftarrow$ & 7 & 2 & 6 & 8009.2769 & 0.0083 \\
\hline 8 & 2 & 6 & $\leftarrow$ & 7 & 2 & 5 & 8205.7571 & 0.0006 \\
\hline 8 & 0 & 8 & $\leftarrow$ & 7 & 0 & 7 & 7848.7802 & -0.0069 \\
\hline 8 & 1 & 7 & $\leftarrow$ & 7 & 1 & 6 & 8173.9968 & 0.0013 \\
\hline \multicolumn{9}{|c|}{$b$-type transitions: } \\
\hline 5 & 1 & 5 & $\leftarrow$ & 4 & 0 & 4 & 5303.7930 & 0.0022 \\
\hline 6 & 1 & 6 & $\leftarrow$ & 5 & 0 & 5 & 6193.6592 & 0.0002 \\
\hline \multirow[t]{2}{*}{6} & 0 & 6 & $\leftarrow$ & 5 & 1 & 5 & 5604.5112 & 0.0001 \\
\hline & & & & & & & RMS & $4.460 \mathrm{kHz}$ \\
\hline
\end{tabular}


Table S19: Measured rotational transitions $\left(\nu_{\text {obs }}\right)$ for the $2 \mathrm{w}(\mathrm{II})-{ }^{18} \mathrm{O} 1$ complex of camphor with two water molecules, and the residuals $\left(\nu_{\mathrm{obs}}-\nu_{\text {calc }}\right)$ for the fit reported in Table S16 (see Figure S4 for oxygen atom numbering).

\begin{tabular}{|c|c|c|c|c|c|c|c|c|}
\hline$J^{\prime}$ & $K_{a}^{\prime}$ & $K_{c}^{\prime}$ & $\leftarrow$ & $J^{\prime \prime}$ & $K_{a}^{\prime \prime}$ & $K_{c}^{\prime \prime}$ & $\nu_{\mathrm{obs}}(\mathrm{MHz})$ & $\nu_{\mathrm{obs}}-\nu_{\mathrm{calc}}$ \\
\hline 3 & 1 & 3 & $\leftarrow$ & 2 & 1 & 2 & 2877.7003 & 0.0007 \\
\hline 3 & 0 & 3 & $\leftarrow$ & 2 & 0 & 2 & 2944.0644 & 0.0016 \\
\hline 3 & 1 & 2 & $\leftarrow$ & 2 & 1 & 1 & 3031.4563 & -0.0009 \\
\hline 4 & 1 & 4 & $\leftarrow$ & 3 & 1 & 3 & 3833.5315 & -0.0005 \\
\hline 4 & 0 & 4 & $\leftarrow$ & 3 & 0 & 3 & 3911.3386 & 0.0015 \\
\hline 4 & 2 & 3 & $\leftarrow$ & 3 & 2 & 2 & 3939.6012 & -0.0009 \\
\hline 4 & 2 & 2 & $\leftarrow$ & 3 & 2 & 1 & 3970.3158 & 0.0006 \\
\hline 4 & 1 & 3 & $\leftarrow$ & 3 & 1 & 2 & 4037.9824 & 0.0003 \\
\hline 5 & 1 & 5 & $\leftarrow$ & 4 & 1 & 4 & 4786.7901 & 0.0009 \\
\hline 5 & 0 & 5 & $\leftarrow$ & 4 & 0 & 4 & 4868.0230 & 0.0019 \\
\hline 5 & 2 & 4 & $\leftarrow$ & 4 & 2 & 3 & 4920.5729 & 0.0021 \\
\hline 5 & 2 & 3 & $\leftarrow$ & 4 & 2 & 2 & 4980.0752 & 0.0004 \\
\hline 5 & 3 & 3 & $\leftarrow$ & 4 & 3 & 2 & 4937.1215 & 0.0023 \\
\hline 5 & 3 & 2 & $\leftarrow$ & 4 & 3 & 1 & 4939.6260 & -0.0029 \\
\hline 5 & 1 & 4 & $\leftarrow$ & 4 & 1 & 3 & 5040.7408 & -0.0020 \\
\hline 6 & 1 & 6 & $\leftarrow$ & 5 & 1 & 5 & 5737.2201 & 0.0022 \\
\hline 6 & 0 & 6 & $\leftarrow$ & 5 & 0 & 5 & 5814.1257 & -0.0014 \\
\hline 6 & 2 & 5 & $\leftarrow$ & 5 & 2 & 4 & 5898.9471 & 0.0021 \\
\hline 6 & 2 & 4 & $\leftarrow$ & 5 & 2 & 3 & 5997.7310 & 0.0008 \\
\hline 6 & 3 & 4 & $\leftarrow$ & 5 & 3 & 3 & 5927.1098 & -0.0026 \\
\hline 6 & 3 & 3 & $\leftarrow$ & 5 & 3 & 2 & 5933.7390 & -0.0032 \\
\hline 6 & 1 & 5 & $\leftarrow$ & 5 & 1 & 4 & 6038.3444 & 0.0010 \\
\hline 7 & 1 & 7 & $\leftarrow$ & 6 & 1 & 6 & 6684.7636 & -0.0045 \\
\hline 7 & 0 & 7 & $\leftarrow$ & 6 & 0 & 6 & 6751.5741 & -0.0010 \\
\hline 7 & 2 & 6 & $\leftarrow$ & 6 & 2 & 5 & 6874.2500 & -0.0048 \\
\hline 7 & 2 & 5 & $\leftarrow$ & 6 & 2 & 4 & 7020.7583 & 0.0000 \\
\hline 7 & 3 & 5 & $\leftarrow$ & 6 & 3 & 4 & 6917.6064 & 0.0005 \\
\hline 7 & 3 & 4 & $\leftarrow$ & 6 & 3 & 3 & 6932.2863 & -0.0007 \\
\hline 7 & 4 & 4 & $\leftarrow$ & 6 & 4 & 3 & 6913.9520 & 0.0089 \\
\hline 7 & 4 & 3 & $\leftarrow$ & 6 & 4 & 2 & 6914.3932 & -0.0053 \\
\hline 7 & 1 & 6 & $\leftarrow$ & 6 & 1 & 5 & 7029.1288 & -0.0012 \\
\hline 8 & 1 & 8 & $\leftarrow$ & 7 & 1 & 7 & 7629.5750 & 0.0008 \\
\hline 8 & 0 & 8 & $\leftarrow$ & 7 & 0 & 7 & 7683.4595 & 0.0021 \\
\hline 8 & 2 & 7 & $\leftarrow$ & 7 & 2 & 6 & 7846.0775 & -0.0044 \\
\hline 8 & 2 & 6 & $\leftarrow$ & 7 & 2 & 5 & 8045.3849 & -0.0036 \\
\hline 8 & 3 & 6 & $\leftarrow$ & 7 & 3 & 5 & 7908.0727 & 0.0032 \\
\hline 8 & 3 & 5 & $\leftarrow$ & 7 & 3 & 4 & 7936.7049 & 0.0047 \\
\hline 8 & 4 & 5 & $\leftarrow$ & 7 & 4 & 4 & 7905.5072 & 0.0039 \\
\hline 8 & 4 & 4 & $\leftarrow$ & 7 & 4 & 3 & 7906.7465 & -0.0001 \\
\hline 8 & 5 & 4 & $\leftarrow$ & 7 & 5 & 3 & 7899.3050 & 0.0078 \\
\hline 8 & 5 & 3 & $\leftarrow$ & 7 & 5 & 2 & 7899.3050 & -0.0162 \\
\hline 8 & 1 & 7 & $\leftarrow$ & 7 & 1 & 6 & 8011.2637 & 0.0025 \\
\hline & & & & & & & RMS & $2.856 \mathrm{kHz}$ \\
\hline
\end{tabular}


Table S20: Measured rotational transitions $\left(\nu_{\text {obs }}\right)$ for the $2 \mathrm{w}(\mathrm{II})-{ }^{18} \mathrm{O} 2$ complex of camphor with two water molecules, and the residuals $\left(\nu_{\mathrm{obs}}-\nu_{\text {calc }}\right)$ for the fit reported in Table S16 (see Figure S4 for oxygen atom numbering).

\begin{tabular}{|c|c|c|c|c|c|c|c|c|}
\hline$J^{\prime}$ & $K_{a}^{\prime}$ & $K_{c}^{\prime}$ & $\leftarrow$ & $J^{\prime \prime}$ & $K_{a}^{\prime \prime}$ & $K_{c}^{\prime \prime}$ & $\nu_{\mathrm{obs}}(\mathrm{MHz})$ & $\nu_{\mathrm{obs}}-\nu_{\mathrm{calc}}$ \\
\hline 3 & 1 & 3 & $\leftarrow$ & 2 & 1 & 2 & 2855.2781 & 0.0040 \\
\hline 3 & 0 & 3 & $\leftarrow$ & 2 & 0 & 2 & 2918.8720 & -0.0009 \\
\hline 3 & 1 & 2 & $\leftarrow$ & 2 & 1 & 1 & 3000.9881 & -0.0039 \\
\hline 4 & 1 & 4 & $\leftarrow$ & 3 & 1 & 3 & 3804.0257 & 0.0023 \\
\hline 4 & 0 & 4 & $\leftarrow$ & 3 & 0 & 3 & 3879.3759 & 0.0000 \\
\hline 4 & 2 & 3 & $\leftarrow$ & 3 & 2 & 2 & 3904.3200 & -0.0005 \\
\hline 4 & 2 & 2 & $\leftarrow$ & 3 & 2 & 1 & 3931.4180 & -0.0011 \\
\hline 4 & 1 & 3 & $\leftarrow$ & 3 & 1 & 2 & 3997.8540 & -0.0025 \\
\hline 5 & 1 & 5 & $\leftarrow$ & 4 & 1 & 4 & 4750.4734 & 0.0009 \\
\hline 5 & 0 & 5 & $\leftarrow$ & 4 & 0 & 4 & 4830.3439 & -0.0013 \\
\hline 5 & 2 & 4 & $\leftarrow$ & 4 & 2 & 3 & 4876.9365 & -0.0034 \\
\hline 5 & 2 & 3 & $\leftarrow$ & 4 & 2 & 2 & 4929.6568 & 0.0001 \\
\hline 5 & 3 & 3 & $\leftarrow$ & 4 & 3 & 2 & 4891.5723 & 0.0022 \\
\hline 5 & 3 & 2 & $\leftarrow$ & 4 & 3 & 1 & 4893.6172 & -0.0039 \\
\hline 5 & 4 & 2 & $\leftarrow$ & 4 & 4 & 1 & 4889.2025 & 0.0138 \\
\hline 5 & 4 & 1 & $\leftarrow$ & 4 & 4 & 0 & 4889.2025 & -0.0093 \\
\hline 5 & 1 & 4 & $\leftarrow$ & 4 & 1 & 3 & 4991.4476 & -0.0008 \\
\hline 6 & 1 & 6 & $\leftarrow$ & 5 & 1 & 5 & 5694.3701 & 0.0004 \\
\hline 6 & 0 & 6 & $\leftarrow$ & 5 & 0 & 5 & 5771.5133 & 0.0015 \\
\hline 6 & 2 & 5 & $\leftarrow$ & 5 & 2 & 4 & 5847.2739 & 0.0012 \\
\hline 6 & 2 & 4 & $\leftarrow$ & 5 & 2 & 3 & 5935.3420 & -0.0001 \\
\hline 6 & 3 & 4 & $\leftarrow$ & 5 & 3 & 3 & 5872.2427 & -0.0007 \\
\hline 6 & 3 & 3 & $\leftarrow$ & 5 & 3 & 2 & 5877.6693 & 0.0004 \\
\hline 6 & 5 & 2 & $\leftarrow$ & 5 & 5 & 1 & 5866.5387 & 0.0000 \\
\hline 6 & 5 & 1 & $\leftarrow$ & 5 & 5 & 0 & 5866.5387 & -0.0007 \\
\hline 6 & 1 & 5 & $\leftarrow$ & 5 & 1 & 4 & 5980.5738 & -0.0024 \\
\hline 7 & 1 & 7 & $\leftarrow$ & 6 & 1 & 6 & 6635.6384 & 0.0027 \\
\hline 7 & 0 & 7 & $\leftarrow$ & 6 & 0 & 6 & 6704.2547 & -0.0037 \\
\hline 7 & 2 & 6 & $\leftarrow$ & 6 & 2 & 5 & 6814.9018 & 0.0032 \\
\hline 7 & 2 & 5 & $\leftarrow$ & 6 & 2 & 4 & 6946.5738 & 0.0031 \\
\hline 7 & 3 & 5 & $\leftarrow$ & 6 & 3 & 4 & 6853.4557 & 0.0013 \\
\hline 7 & 3 & 4 & $\leftarrow$ & 6 & 3 & 3 & 6865.5011 & 0.0050 \\
\hline 7 & 4 & 4 & $\leftarrow$ & 6 & 4 & 3 & 6849.8550 & 0.0060 \\
\hline 7 & 4 & 3 & $\leftarrow$ & 6 & 4 & 2 & 6850.1881 & -0.0064 \\
\hline 7 & 5 & 3 & $\leftarrow$ & 6 & 5 & 2 & 6846.0248 & 0.0043 \\
\hline 7 & 5 & 2 & $\leftarrow$ & 6 & 5 & 1 & 6846.0248 & 0.0001 \\
\hline 7 & 1 & 6 & $\leftarrow$ & 6 & 1 & 5 & 6963.8300 & 0.0020 \\
\hline 8 & 1 & 8 & $\leftarrow$ & 7 & 1 & 7 & 7574.3517 & -0.0009 \\
\hline 8 & 0 & 8 & $\leftarrow$ & 7 & 0 & 7 & 7631.1640 & -0.0027 \\
\hline 8 & 2 & 7 & $\leftarrow$ & 7 & 2 & 6 & 7779.4375 & 0.0005 \\
\hline 8 & 2 & 6 & $\leftarrow$ & 7 & 2 & 5 & 7960.1803 & -0.0015 \\
\hline 8 & 3 & 6 & $\leftarrow$ & 7 & 3 & 5 & 7834.7717 & -0.0002 \\
\hline 8 & 3 & 5 & $\leftarrow$ & 7 & 3 & 4 & 7858.3387 & -0.0033 \\
\hline 8 & 4 & 5 & $\leftarrow$ & 7 & 4 & 4 & 7831.8175 & -0.0040 \\
\hline 8 & 4 & 4 & $\leftarrow$ & 7 & 4 & 3 & 7832.7620 & -0.0038 \\
\hline 8 & 5 & 4 & $\leftarrow$ & 7 & 5 & 3 & 7826.3086 & 0.0103 \\
\hline 8 & 5 & 3 & $\leftarrow$ & 7 & 5 & 2 & 7826.3086 & -0.0066 \\
\hline \multirow[t]{2}{*}{8} & 1 & 7 & $\leftarrow$ & 7 & 1 & 6 & 7939.6146 & 0.0055 \\
\hline & & & & & & & RMS & $2.795 \mathrm{kHz}$ \\
\hline
\end{tabular}


Table S21: Measured rotational transitions $\left(\nu_{\mathrm{obs}}\right)$ for the $2 \mathrm{w}(\mathrm{II})-{ }^{18} \mathrm{O} 1^{18} \mathrm{O} 2$ complex of camphor with two water molecules, and the residuals $\left(\nu_{\text {obs }}-\nu_{\text {calc }}\right)$ for the fit reported in Table S16 (see Figure S4 for oxygen atom numbering).

\begin{tabular}{|c|c|c|c|c|c|c|c|c|}
\hline$J^{\prime}$ & $K_{a}^{\prime}$ & $K_{c}^{\prime}$ & $\leftarrow$ & $J^{\prime \prime}$ & $K_{a}^{\prime \prime}$ & $K_{c}^{\prime \prime}$ & $\nu_{\mathrm{obs}}(\mathrm{MHz})$ & $\nu_{\mathrm{obs}}-\nu_{\mathrm{calc}}$ \\
\hline 3 & 1 & 3 & $\leftarrow$ & 2 & 1 & 2 & 2798.5179 & 0.0009 \\
\hline 3 & 0 & 3 & $\leftarrow$ & 2 & 0 & 2 & 2862.6816 & 0.0006 \\
\hline 3 & 1 & 2 & $\leftarrow$ & 2 & 1 & 1 & 2945.9610 & 0.0000 \\
\hline 4 & 1 & 4 & $\leftarrow$ & 3 & 1 & 3 & 3728.2570 & 0.0024 \\
\hline 4 & 0 & 4 & $\leftarrow$ & 3 & 0 & 3 & 3804.0630 & -0.0044 \\
\hline 4 & 2 & 3 & $\leftarrow$ & 3 & 2 & 2 & 3829.7983 & -0.0018 \\
\hline 4 & 2 & 2 & $\leftarrow$ & 3 & 2 & 1 & 3857.7566 & -0.0003 \\
\hline 4 & 1 & 3 & $\leftarrow$ & 3 & 1 & 2 & 3924.3609 & -0.0037 \\
\hline 5 & 1 & 5 & $\leftarrow$ & 4 & 1 & 4 & 4655.6350 & 0.0078 \\
\hline 5 & 0 & 5 & $\leftarrow$ & 4 & 0 & 4 & 4735.6702 & 0.0005 \\
\hline 5 & 2 & 4 & $\leftarrow$ & 4 & 2 & 3 & 4783.6750 & -0.0031 \\
\hline 5 & 2 & 3 & $\leftarrow$ & 4 & 2 & 2 & 4838.0077 & 0.0008 \\
\hline 5 & 3 & 3 & $\leftarrow$ & 4 & 3 & 2 & 4798.7625 & -0.0002 \\
\hline 5 & 3 & 2 & $\leftarrow$ & 4 & 3 & 1 & 4800.9210 & -0.0021 \\
\hline 5 & 4 & 2 & $\leftarrow$ & 4 & 4 & 1 & 4796.3332 & 0.0098 \\
\hline 5 & 4 & 1 & $\leftarrow$ & 4 & 4 & 0 & 4796.3332 & -0.0151 \\
\hline 5 & 1 & 4 & $\leftarrow$ & 4 & 1 & 3 & 4899.3845 & 0.0051 \\
\hline 6 & 1 & 6 & $\leftarrow$ & 5 & 1 & 5 & 5580.3821 & -0.0007 \\
\hline 6 & 0 & 6 & $\leftarrow$ & 5 & 0 & 5 & 5657.2864 & 0.0002 \\
\hline 6 & 2 & 5 & $\leftarrow$ & 5 & 2 & 4 & 5735.1950 & -0.0020 \\
\hline 6 & 2 & 4 & $\leftarrow$ & 5 & 2 & 3 & 5825.8078 & -0.0029 \\
\hline 6 & 3 & 4 & $\leftarrow$ & 5 & 3 & 3 & 5760.9227 & -0.0023 \\
\hline 6 & 3 & 3 & $\leftarrow$ & 5 & 3 & 2 & 5766.6371 & -0.0003 \\
\hline 6 & 1 & 5 & $\leftarrow$ & 5 & 1 & 4 & 5869.7692 & 0.0043 \\
\hline 7 & 1 & 7 & $\leftarrow$ & 6 & 1 & 6 & 6502.4543 & 0.0047 \\
\hline 7 & 0 & 7 & $\leftarrow$ & 6 & 0 & 6 & 6570.4339 & 0.0021 \\
\hline 7 & 2 & 6 & $\leftarrow$ & 6 & 2 & 5 & 6683.9198 & -0.0046 \\
\hline 7 & 2 & 5 & $\leftarrow$ & 6 & 2 & 4 & 6819.1131 & 0.0004 \\
\hline 7 & 3 & 5 & $\leftarrow$ & 6 & 3 & 4 & 6723.6197 & 0.0037 \\
\hline 7 & 3 & 4 & $\leftarrow$ & 6 & 3 & 3 & 6736.2885 & 0.0013 \\
\hline 7 & 4 & 4 & $\leftarrow$ & 6 & 4 & 3 & 6719.9989 & 0.0014 \\
\hline 7 & 4 & 3 & $\leftarrow$ & 6 & 4 & 2 & 6720.3651 & -0.0036 \\
\hline 7 & 5 & 3 & $\leftarrow$ & 6 & 5 & 2 & 6716.0517 & 0.0019 \\
\hline 7 & 5 & 2 & $\leftarrow$ & 6 & 5 & 1 & 6716.0517 & -0.0026 \\
\hline 7 & 1 & 6 & $\leftarrow$ & 6 & 1 & 5 & 6834.0524 & 0.0009 \\
\hline 8 & 1 & 8 & $\leftarrow$ & 7 & 1 & 7 & 7421.9180 & -0.0044 \\
\hline 8 & 0 & 8 & $\leftarrow$ & 7 & 0 & 7 & 7477.8151 & -0.0016 \\
\hline 8 & 2 & 7 & $\leftarrow$ & 7 & 2 & 6 & 7629.4671 & -0.0038 \\
\hline 8 & 2 & 6 & $\leftarrow$ & 7 & 2 & 5 & 7814.6008 & 0.0007 \\
\hline 8 & 3 & 6 & $\leftarrow$ & 7 & 3 & 5 & 7686.3850 & 0.0043 \\
\hline 8 & 3 & 5 & $\leftarrow$ & 7 & 3 & 4 & 7711.1567 & -0.0032 \\
\hline 8 & 4 & 5 & $\leftarrow$ & 7 & 4 & 4 & 7683.5275 & 0.0036 \\
\hline 8 & 4 & 4 & $\leftarrow$ & 7 & 4 & 3 & 7684.5404 & 0.0019 \\
\hline \multirow[t]{2}{*}{8} & 1 & 7 & $\leftarrow$ & 7 & 1 & 6 & 7790.5841 & -0.0007 \\
\hline & & & & & & & RMS & $2.926 \mathrm{kHz}$ \\
\hline
\end{tabular}




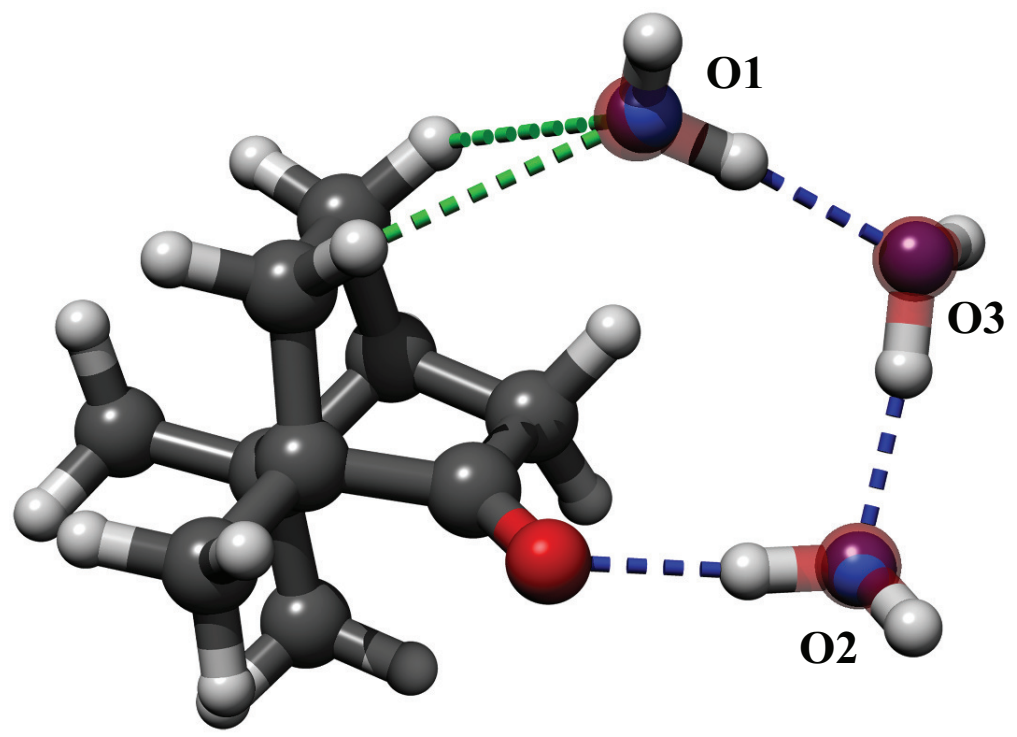

$3 \mathbf{w}$

(a)

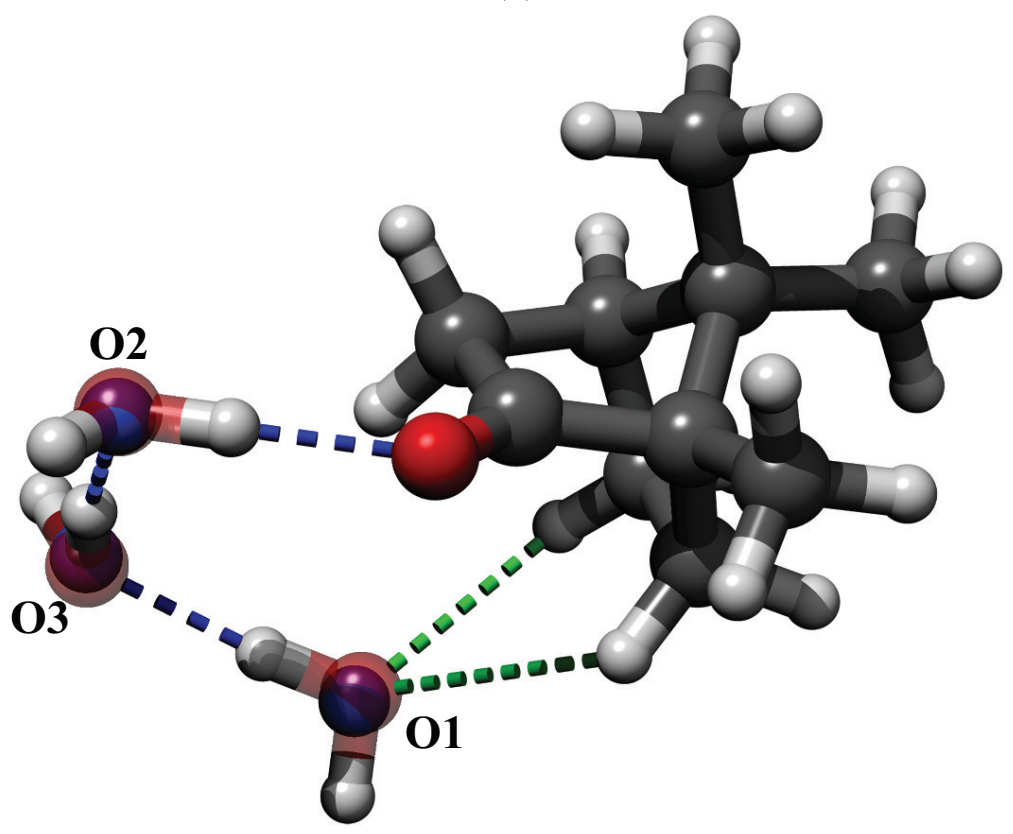

3w

(b)

Figure S5: Calculated structure (MP2/6-311++G(d,p)) for the camphor complex with three water molecules $(3 \mathrm{w})$. Oxygen atom numbering used for the $\mathrm{H}_{2}{ }^{18} \mathrm{O}$ isotopologues of the complex is shown. The blue/purple spheres represent the experimental oxygen positions from the Kraitchman analysis. Two orientations (a, b) of the $3 \mathrm{w}-$ complex are depicted for better visualisation of the intermolecular bonds. Strong hydrogen bonds are depicted in blue, dispersive interactions are shown in green. 
Table S22: Experimentally determined rotational and quartic centrifugal distortion constants for the normal and the $\mathrm{H}_{2}{ }^{18} \mathrm{O}$ substituted species of the complex of camphor with three water molecules (see Figure S5 for oxygen atom numbering).

\begin{tabular}{|c|c|c|c|c|}
\hline & normal & ${ }^{18} \mathrm{O} 1$ & ${ }^{18} \mathrm{O} 2$ & ${ }^{18} \mathrm{O} 3$ \\
\hline$A(\mathrm{MHz})$ & $893.24443(30)$ & $877.54097(45)$ & $880.89566(31)$ & $891.03346(42)$ \\
\hline$B(\mathrm{MHz})$ & $401.10981(12)$ & $397.23648(16)$ & $394.51613(13)$ & $390.17450(17)$ \\
\hline$C(\mathrm{MHz})$ & $356.27688(12)$ & $351.24670(18)$ & $349.17175(15)$ & $347.68162(20)$ \\
\hline$\Delta_{J}(\mathrm{kHz})$ & $0.09431(71)$ & $0.0905(10)$ & $0.08984(91)$ & 0.0972(11) \\
\hline$\Delta_{J K}(\mathrm{kHz})$ & $-0.1721(72)$ & $-0.134(10)$ & $-0.1867(35)$ & $-0.2094(96)$ \\
\hline$N_{\text {lines }}$ & 63 & 72 & 69 & 67 \\
\hline$\sigma(\mathrm{kHz})$ & 3.45 & 5.19 & 4.54 & 5.23 \\
\hline$P_{a}\left(\mathrm{u} \AA^{2}\right)$ & $1056.3366(3)$ & $1067.5742(5)$ & $1077.3322(4)$ & $1090.8247(5)$ \\
\hline$P_{b}\left(\mathrm{u} \AA^{2}\right)$ & $362.1640(3)$ & $371.2407(5)$ & $370.0328(4)$ & $362.7436(5)$ \\
\hline \multirow[t]{2}{*}{$P_{c}\left(\mathrm{u} \AA^{2}\right)$} & $203.6150(3)$ & $204.6628(5)$ & $203.6775(4)$ & $204.4393(5)$ \\
\hline & ${ }^{18} \mathrm{O} 1+{ }^{18} \mathrm{O} 2$ & ${ }^{18} \mathrm{O} 1+{ }^{18} \mathrm{O} 3$ & ${ }^{18} \mathrm{O} 2+{ }^{18} \mathrm{O} 3$ & ${ }^{18} \mathrm{O} 1+{ }^{18} \mathrm{O} 2+{ }^{18} \mathrm{O} 3$ \\
\hline$A(\mathrm{MHz})$ & $864.79819(35)$ & $875.54001(31)$ & $878.44926(44)$ & $862.61258(36)$ \\
\hline$B(\mathrm{MHz})$ & $390.97490(15)$ & $386.57034(13)$ & $384.13208(16)$ & $380.82336(15)$ \\
\hline$C(\mathrm{MHz})$ & $344.36329(16)$ & $342.99939(15)$ & $341.01994(16)$ & $336.53504(16)$ \\
\hline$\Delta_{J}(\mathrm{kHz})$ & $0.08766(96)$ & $0.09262(86)$ & $0.09192(94)$ & $0.08958(94)$ \\
\hline$\Delta_{J K}(\mathrm{kHz})$ & $-0.1580(71)$ & $-0.1820(47)$ & $-0.1780(81)$ & $-0.1789(76)$ \\
\hline$N_{\text {lines }}$ & 71 & 73 & 68 & 72 \\
\hline$\sigma(\mathrm{kHz})$ & 5.08 & 4.37 & 4.87 & 4.86 \\
\hline$P_{a}\left(\mathrm{u} \AA^{2}\right)$ & $1087.8990(4)$ & $1101.7656(4)$ & $1111.1468(5)$ & $1121.4560(5)$ \\
\hline$P_{b}\left(\mathrm{u} \AA^{2}\right)$ & $379.6761(4)$ & $371.6451(4)$ & $370.8163(5)$ & $380.2568(5)$ \\
\hline$P_{c}\left(\mathrm{u} \AA^{2}\right)$ & $204.7133(4)$ & $205.5746(4)$ & $204.4917(5)$ & $205.6133(5)$ \\
\hline
\end{tabular}


Table S23: Experimental coordinates $(\AA)$ of the water oxygen atoms in the camphor complex $(3 \mathrm{w})$ with three water molecules, compared with the results from ab initio calculations.

\begin{tabular}{|c|c|c|c|c|c|}
\hline & $r_{\mathrm{s}}^{a}$ & $r_{0}^{b}$ & $\mathrm{Calc}^{c}$ & Calc $^{d}$ & $\mathrm{Calc}^{e}$ \\
\hline \multicolumn{6}{|c|}{ O1 } \\
\hline$a$ & $2.3624(6)$ & $2.3623(10)$ & 2.2587 & - & 2.2795 \\
\hline$b$ & $2.1486(7)$ & $2.1475(11)$ & 2.1158 & - & 2.1470 \\
\hline$c$ & $0.7522(20)$ & $0.7580(29)$ & 0.6560 & - & 0.6937 \\
\hline \multicolumn{6}{|c|}{$\mathrm{O} 2$} \\
\hline$a$ & $3.2337(5)$ & $3.2329(7)$ & 3.2833 & - & 3.2203 \\
\hline$b$ & $2.0206(8)$ & $-2.0197(11)$ & -1.9761 & - & -1.9940 \\
\hline$c$ & $0.1848(82)$ & $-0.2133(30)$ & -0.2553 & - & -0.2372 \\
\hline \multicolumn{6}{|c|}{ O3 } \\
\hline$a$ & $4.1646(4)$ & $4.1653(6)$ & 4.2941 & - & 4.1782 \\
\hline$b$ & $0.5527(27)$ & $0.5407(27)$ & 0.5681 & - & 0.5445 \\
\hline$c$ & $0.6587(23)$ & $-0.6606(37)$ & -0.4815 & - & -0.5556 \\
\hline
\end{tabular}

${ }^{a}$ Determined using Kraitchman equations, which result in only the absolute value, while uncertainties were estimated with the Costain criterion.

${ }^{b}$ Obtained from the least squares $r_{0}$ fit where the cited uncertainties only reflect those in the explicit parameters of the fit.

${ }^{c} \mathrm{MP} 2 / 6-311++\mathrm{G}(\mathrm{d}, \mathrm{p})$ calculation.

${ }^{d}$ M06-2X/6-311++G(d,p) calculation.

${ }^{r}$ B3LYP-D3/aug-cc-pVTZ calculation. 
Table S24: Measured rotational transitions $\left(\nu_{\mathrm{obs}}\right)$ for the $3 \mathrm{w}$ complex of camphor with three water molecules (main isotopologues), and the residuals $\left(\nu_{\text {obs }}-\nu_{\text {calc }}\right)$ for the fit reported in Table 1.

\begin{tabular}{ccccccccc}
\hline$J^{\prime}$ & $K_{a}^{\prime}$ & $K_{c}^{\prime}$ & $\leftarrow$ & $J^{\prime \prime}$ & $K_{a}^{\prime \prime}$ & $K_{c}^{\prime \prime}$ & $\nu_{\mathrm{obs}}(\mathrm{MHz})$ & $\nu_{\mathrm{obs}}-\nu_{\text {calc }}$ \\
\hline
\end{tabular}

$a$-type transitions:

$\begin{array}{ccccccccc}3 & 1 & 3 & \leftarrow & 2 & 1 & 2 & 2203.1310 & 0.0018 \\ 3 & 0 & 3 & \leftarrow & 2 & 0 & 2 & 2260.5276 & -0.0020 \\ 3 & 1 & 2 & \leftarrow & 2 & 1 & 1 & 2337.5050 & -0.0033 \\ & & & & & & & & \\ 4 & 1 & 4 & \leftarrow & 3 & 1 & 3 & 2934.3496 & 0.0001 \\ 4 & 0 & 4 & \leftarrow & 3 & 0 & 3 & 3000.9879 & -0.0009 \\ 4 & 2 & 2 & \leftarrow & 3 & 2 & 1 & 3055.7795 & -0.0004 \\ 4 & 1 & 3 & \leftarrow & 3 & 1 & 2 & 3112.9646 & -0.0007 \\ 5 & 1 & 5 & \leftarrow & 4 & 1 & 4 & 3663.1987 & -0.0014 \\ 5 & 0 & 5 & \leftarrow & 4 & 0 & 4 & 3731.7966 & -0.0013 \\ 5 & 2 & 4 & \leftarrow & 4 & 2 & 3 & 3780.3955 & 0.0010 \\ 5 & 3 & 2 & \leftarrow & 4 & 3 & 1 & 3798.2477 & -0.0021 \\ 5 & 2 & 3 & \leftarrow & 4 & 2 & 2 & 3835.4484 & -0.0020 \\ & & & & & & & & \\ 6 & 1 & 6 & \leftarrow & 5 & 1 & 5 & 4389.4708 & -0.0013 \\ 6 & 0 & 6 & \leftarrow & 5 & 0 & 5 & 4453.2340 & -0.0004 \\ 6 & 2 & 5 & \leftarrow & 5 & 2 & 4 & 4531.1271 & 0.0056 \\ 6 & 3 & 4 & \leftarrow & 5 & 3 & 3 & 4557.2012 & 0.0020 \\ 6 & 3 & 3 & \leftarrow & 5 & 3 & 2 & 4563.7703 & 0.0015 \\ 6 & 2 & 4 & \leftarrow & 5 & 2 & 3 & 4621.9774 & -0.0029 \\ 6 & 1 & 5 & \leftarrow & 5 & 1 & 4 & 4651.8994 & 0.0004 \\ & & & & & & & & \\ 7 & 1 & 7 & \leftarrow & 6 & 1 & 6 & 5113.1493 & -0.0014 \\ 7 & 0 & 7 & \leftarrow & 6 & 0 & 6 & 5167.3487 & 0.0018 \\ 7 & 2 & 6 & \leftarrow & 6 & 2 & 5 & 5278.9978 & -0.0017 \\ 7 & 3 & 5 & \leftarrow & 6 & 3 & 4 & 5319.0091 & 0.0023 \\ 7 & 3 & 4 & \leftarrow & 6 & 3 & 3 & 5333.5233 & 0.0021 \\ 7 & 1 & 6 & \leftarrow & 6 & 1 & 5 & 5412.4578 & -0.0023 \\ 7 & 2 & 5 & \leftarrow & 6 & 2 & 4 & 5412.7447 & -0.0037 \\ & & & & & & & & \\ 8 & 1 & 8 & \leftarrow & 7 & 1 & 7 & 5834.3911 & -0.0006 \\ 8 & 0 & 8 & \leftarrow & 7 & 0 & 7 & 5877.0865 & -0.0010 \\ 8 & 2 & 7 & \leftarrow & 7 & 2 & 6 & 6023.6502 & -0.0011 \\ 8 & 4 & 5 & \leftarrow & 7 & 4 & 4 & 6078.9640 & 0.0031 \\ 8 & 4 & 4 & \leftarrow & 7 & 4 & 3 & 6080.2709 & -0.0037 \\ 8 & 3 & 6 & \leftarrow & 7 & 3 & 5 & 6080.6585 & 0.0021 \\ 8 & 1 & 7 & \leftarrow & 7 & 1 & 6 & 6164.8291 & 0.0011 \\ 8 & 2 & 6 & \leftarrow & 7 & 2 & 5 & 6204.1485 & -0.0017 \\ & & & & & & & & \\ 9 & 1 & 9 & \leftarrow & 8 & 1 & 8 & 6553.4725 & -0.0046 \\ 9 & 0 & 9 & \leftarrow & 8 & 0 & 8 & 6585.1118 & -0.0009\end{array}$




$\begin{array}{ccccccccc}9 & 4 & 6 & \leftarrow & 8 & 4 & 5 & 6843.0661 & 0.0016 \\ 9 & 4 & 5 & \leftarrow & 8 & 4 & 4 & 6846.1745 & -0.0046 \\ 9 & 3 & 6 & \leftarrow & 8 & 3 & 5 & 6890.9852 & -0.0021 \\ 9 & 1 & 8 & \leftarrow & 8 & 1 & 7 & 6907.3994 & 0.0010 \\ 9 & 2 & 7 & \leftarrow & 8 & 2 & 6 & 6992.7573 & 0.0005 \\ & & & & & & & & \\ 10 & 1 & 10 & \leftarrow & 9 & 1 & 9 & 7270.7632 & 0.0053 \\ 10 & 0 & 10 & \leftarrow & 9 & 0 & 9 & 7293.1116 & 0.0003 \\ 10 & 2 & 9 & \leftarrow & 5 & 2 & 8 & 7502.2021 & 0.0041 \\ 10 & 1 & 9 & \leftarrow & 9 & 1 & 8 & 7639.1861 & -0.0063 \\ 10 & 3 & 7 & \leftarrow & 5 & 3 & 6 & 7680.4821 & 0.0026 \\ 10 & 2 & 8 & \leftarrow & \leftarrow & 2 & 7 & 7775.9378 & -0.0009\end{array}$

$c$-type transitions:

\begin{tabular}{ccccccccc}
3 & 2 & 1 & $\leftarrow$ & 2 & 1 & 1 & 3763.1166 & 0.0098 \\
4 & 2 & 2 & $\leftarrow$ & 3 & 1 & 2 & 4481.3836 & 0.0052 \\
3 & 3 & 0 & $\leftarrow$ & 2 & 2 & 0 & 4843.8884 & 0.0022 \\
3 & 3 & 1 & $\leftarrow$ & 2 & 2 & 1 & 4846.6880 & -0.0041 \\
5 & 2 & 3 & $\leftarrow$ & 4 & 1 & 3 & 5203.8704 & 0.0069 \\
5 & 2 & 4 & $\leftarrow$ & 4 & 1 & 4 & 5553.2347 & 0.0087 \\
6 & 1 & 5 & $\leftarrow$ & 5 & 0 & 5 & 5578.6566 & -0.0009 \\
4 & 3 & 1 & $\leftarrow$ & 3 & 2 & 1 & 5595.8675 & -0.0039 \\
4 & 3 & 2 & $\leftarrow$ & 3 & 2 & 2 & 5609.5770 & -0.0048 \\
6 & 2 & 4 & $\leftarrow$ & 5 & 1 & 4 & 5940.9753 & 0.0057 \\
5 & 3 & 2 & $\leftarrow$ & 4 & 2 & 2 & 6338.3371 & -0.0042 \\
5 & 3 & 3 & $\leftarrow$ & 4 & 2 & 3 & 6378.0833 & -0.0077 \\
6 & 3 & 3 & $\leftarrow$ & 5 & 2 & 3 & 7066.6574 & -0.0023 \\
6 & 3 & 4 & $\leftarrow$ & 5 & 2 & 4 & 7154.8953 & -0.0004 \\
8 & 2 & 6 & $\leftarrow$ & 7 & 1 & 6 & 7493.5120 & 0.0029 \\
7 & 3 & 4 & $\leftarrow$ & 6 & 2 & 4 & 7778.2037 & 0.0032 \\
& & & & & & & & \\
& & & & & & & RMS & $3.451 \mathrm{kHz}$ \\
\hline
\end{tabular}


Table S25: Measured rotational transitions $\left(\nu_{\mathrm{obs}}\right)$ for the $3 \mathrm{w}-{ }^{18} \mathrm{O} 1$ complex of camphor with three water molecules, and the residuals $\left(\nu_{\mathrm{obs}}-\nu_{\text {calc }}\right)$ for the fit reported in Table S22 (see Figure S5 for oxygen atom numbering).

\begin{tabular}{ccccccccc}
\hline$J^{\prime}$ & $K_{a}^{\prime}$ & $K_{c}^{\prime}$ & $\leftarrow$ & $J^{\prime \prime}$ & $K_{a}^{\prime \prime}$ & $K_{c}^{\prime \prime}$ & $\nu_{\mathrm{obs}}(\mathrm{MHz})$ & $\nu_{\mathrm{obs}}-\nu_{\text {calc }}$ \\
\hline
\end{tabular}

$a$-type transitions:

$\begin{array}{ccccccccc}3 & 1 & 3 & \leftarrow & 2 & 1 & 2 & 2174.5483 & -0.0038 \\ 3 & 1 & 2 & \leftarrow & 2 & 1 & 1 & 2312.3854 & -0.0011 \\ & & & & & & & & \\ 4 & 1 & 4 & \leftarrow & 3 & 1 & 3 & 2896.0232 & 0.0005 \\ 4 & 0 & 4 & \leftarrow & 3 & 0 & 3 & 2963.2897 & -0.0014 \\ 4 & 2 & 3 & \leftarrow & 3 & 2 & 2 & 2991.4595 & -0.0039 \\ 4 & 1 & 3 & \leftarrow & 3 & 1 & 2 & 3079.1725 & -0.0020 \\ & & & & & & & & \\ 5 & 0 & 5 & \leftarrow & 4 & 0 & 4 & 3683.4503 & 0.0060 \\ 5 & 2 & 4 & \leftarrow & 4 & 2 & 3 & 3735.3844 & -0.0014 \\ 5 & 3 & 3 & \leftarrow & 4 & 3 & 2 & 3751.8532 & 0.0022 \\ 5 & 3 & 2 & \leftarrow & 4 & 3 & 1 & 3754.6580 & -0.0002 \\ 5 & 2 & 3 & \leftarrow & 4 & 2 & 2 & 3794.2726 & -0.0012 \\ 5 & 1 & 4 & \leftarrow & 4 & 1 & 3 & 3842.1116 & -0.0028 \\ & & & & & & & & \\ 6 & 1 & 6 & \leftarrow & 5 & 1 & 5 & 4331.2007 & -0.0003 \\ 6 & 0 & 6 & \leftarrow & 5 & 0 & 5 & 4393.9256 & -0.0015 \\ 6 & 2 & 5 & \leftarrow & 5 & 2 & 4 & 4476.7126 & -0.0005 \\ 6 & 3 & 4 & \leftarrow & 5 & 3 & 3 & 4504.6061 & 0.0022 \\ 6 & 3 & 3 & \leftarrow & 5 & 3 & 2 & 4512.0114 & 0.0103 \\ 6 & 2 & 4 & \leftarrow & 5 & 2 & 3 & 4573.4324 & -0.0027 \\ 6 & 1 & 5 & \leftarrow & 5 & 1 & 4 & 4599.7375 & -0.0034 \\ & & & & & & & & \\ 7 & 1 & 7 & \leftarrow & 6 & 1 & 6 & 5044.7181 & 0.0008 \\ 7 & 0 & 7 & \leftarrow & 6 & 0 & 6 & 5097.1370 & 0.0015 \\ 7 & 2 & 6 & \leftarrow & 6 & 2 & 5 & 5214.9870 & 0.0001 \\ 7 & 4 & 4 & \leftarrow & 6 & 4 & 3 & 5254.7397 & 0.0028 \\ 7 & 4 & 3 & \leftarrow & 6 & 4 & 2 & 5255.3035 & -0.0026 \\ 7 & 3 & 5 & \leftarrow & 6 & 3 & 4 & 5257.6747 & -0.0026 \\ 7 & 3 & 4 & \leftarrow & 6 & 3 & 3 & 5273.9880 & -0.0007 \\ 7 & 1 & 6 & \leftarrow & 6 & 1 & 5 & 5350.3231 & -0.0029 \\ 7 & 2 & 5 & \leftarrow & 6 & 2 & 4 & 5356.5319 & -0.0082 \\ & & & & & & & & \\ 8 & 1 & 8 & \leftarrow & 7 & 1 & 7 & 5755.7188 & 0.0042 \\ 8 & 0 & 8 & \leftarrow & 7 & 0 & 7 & 5796.2719 & 0.0047 \\ 8 & 2 & 7 & \leftarrow & 7 & 2 & 6 & 5949.8143 & 0.0014 \\ 8 & 4 & 5 & \leftarrow & 7 & 4 & 4 & 6009.1830 & -0.0072 \\ 8 & 3 & 6 & \leftarrow & 7 & 3 & 5 & 6010.4803 & -0.0012 \\ 8 & 4 & 4 & \leftarrow & 7 & 4 & 3 & 6010.7359 & -0.0059 \\ 8 & 3 & 5 & \leftarrow & 7 & 3 & 4 & 6042.0782 & 0.0034 \\ 8 & 1 & 7 & \leftarrow & 7 & 1 & 6 & 6092.0035 & -0.0025\end{array}$




$\begin{array}{ccccccccc}8 & 2 & 6 & \leftarrow & 7 & 2 & 5 & 6139.6668 & -0.0070 \\ 9 & 1 & 9 & \leftarrow & 8 & 1 & 8 & 6464.5153 & 0.0007 \\ 9 & 0 & 9 & \leftarrow & 8 & 0 & 8 & 6494.0161 & -0.0024 \\ 9 & 2 & 8 & \leftarrow & 8 & 2 & 7 & 6680.8899 & -0.0008 \\ 9 & 3 & 7 & \leftarrow & 8 & 3 & 6 & 6762.3085 & 0.0057 \\ 9 & 3 & 6 & \leftarrow & 8 & 3 & 5 & 6817.4833 & 0.0001 \\ 9 & 1 & 8 & \leftarrow & 8 & 1 & 7 & 6823.1316 & -0.0016 \\ 9 & 2 & 7 & \leftarrow & 8 & 2 & 6 & 6919.2746 & -0.0065 \\ & & & & & & & & \\ 10 & 1 & 10 & \leftarrow & 9 & 1 & 9 & 7171.5065 & 0.0035 \\ 10 & 0 & 10 & \leftarrow & 9 & 0 & 9 & 7191.9821 & -0.0008 \\ 10 & 2 & 9 & \leftarrow & 9 & 2 & 8 & 7408.0469 & 0.0022 \\ 10 & 3 & 8 & \leftarrow & 9 & 3 & 7 & 7512.3772 & 0.0038 \\ 10 & 4 & 7 & \leftarrow & 9 & 4 & 6 & 7521.5207 & 0.0099 \\ 10 & 1 & 9 & \leftarrow & 9 & 1 & 8 & 7542.8681 & -0.0056 \\ 10 & 2 & 8 & \leftarrow & 9 & 2 & 7 & 7692.7111 & -0.0047\end{array}$

$c$-type transitions:

\begin{tabular}{ccccccccc}
3 & 1 & 2 & $\leftarrow$ & 2 & 0 & 2 & 2887.8083 & -0.0100 \\
2 & 2 & 0 & $\leftarrow$ & 1 & 1 & 0 & 2987.0237 & 0.0071 \\
4 & 1 & 3 & $\leftarrow$ & 3 & 0 & 3 & 3734.0514 & 0.0078 \\
4 & 2 & 2 & $\leftarrow$ & 3 & 1 & 2 & 4411.6911 & 0.0042 \\
4 & 2 & 3 & $\leftarrow$ & 3 & 1 & 3 & 4641.2528 & 0.0133 \\
3 & 3 & 1 & $\leftarrow$ & 2 & 2 & 1 & 4763.8440 & -0.0076 \\
5 & 2 & 3 & $\leftarrow$ & 4 & 1 & 3 & 5126.7936 & 0.0073 \\
4 & 3 & 1 & $\leftarrow$ & 3 & 2 & 1 & 5503.5381 & -0.0029 \\
4 & 3 & 2 & $\leftarrow$ & 3 & 2 & 2 & 5518.2320 & -0.0042 \\
6 & 1 & 5 & $\leftarrow$ & 5 & 0 & 5 & 5529.1626 & -0.0011 \\
6 & 2 & 4 & $\leftarrow$ & 5 & 1 & 4 & 5858.1142 & 0.0073 \\
5 & 3 & 2 & $\leftarrow$ & 4 & 2 & 2 & 6236.1190 & -0.0032 \\
5 & 3 & 3 & $\leftarrow$ & 4 & 2 & 3 & 6278.6139 & -0.0099 \\
6 & 2 & 5 & $\leftarrow$ & 5 & 1 & 5 & 6342.3585 & 0.0156 \\
7 & 2 & 5 & $\leftarrow$ & 6 & 1 & 5 & 6614.9110 & 0.0050 \\
6 & 3 & 3 & $\leftarrow$ & 5 & 2 & 3 & 6953.8508 & 0.0014 \\
6 & 3 & 4 & $\leftarrow$ & 5 & 2 & 4 & 7047.8465 & 0.0046 \\
7 & 2 & 6 & $\leftarrow$ & 6 & 1 & 6 & 7226.1332 & 0.0044 \\
8 & 2 & 6 & $\leftarrow$ & 7 & 1 & 6 & 7404.2527 & -0.0010 \\
7 & 3 & 4 & $\leftarrow$ & 6 & 2 & 4 & 7654.3974 & -0.0055 \\
7 & 3 & 5 & $\leftarrow$ & 6 & 2 & 5 & 7828.7972 & -0.0089 \\
& & & & & & & & \\
& & & & & & & $\mathrm{RMS}$ & $5.193 \mathrm{kHz}$ \\
\hline
\end{tabular}


Table S26: Measured rotational transitions $\left(\nu_{\mathrm{obs}}\right)$ for the $3 \mathrm{w}-{ }^{18} \mathrm{O} 2$ complex of camphor with three water molecules, and the residuals $\left(\nu_{\mathrm{obs}}-\nu_{\text {calc }}\right)$ for the fit reported in Table S22 (see Figure S5 for oxygen atom numbering).

\begin{tabular}{ccccccccc}
\hline$J^{\prime}$ & $K_{a}^{\prime}$ & $K_{c}^{\prime}$ & $\leftarrow$ & $J^{\prime \prime}$ & $K_{a}^{\prime \prime}$ & $K_{c}^{\prime \prime}$ & $\nu_{\mathrm{obs}}(\mathrm{MHz})$ & $\nu_{\mathrm{obs}}-\nu_{\text {calc }}$ \\
\hline
\end{tabular}

$a$-type transitions:

$\begin{array}{ccccccccc}3 & 1 & 3 & \leftarrow & 2 & 1 & 2 & 2161.2127 & 0.0055 \\ 3 & 0 & 3 & \leftarrow & 2 & 0 & 2 & 2219.0376 & -0.0056 \\ 3 & 1 & 2 & \leftarrow & 2 & 1 & 1 & 2297.1133 & -0.0005 \\ & & & & & & & & \\ 4 & 1 & 4 & \leftarrow & 3 & 1 & 3 & 2878.3508 & -0.0022 \\ 4 & 0 & 4 & \leftarrow & 3 & 0 & 3 & 2945.2600 & 0.0012 \\ 4 & 2 & 3 & \leftarrow & 3 & 2 & 2 & 2972.3803 & 0.0010 \\ 4 & 2 & 2 & \leftarrow & 3 & 2 & 1 & 3001.8437 & -0.0009 \\ 4 & 1 & 3 & \leftarrow & 3 & 1 & 2 & 3058.9716 & -0.0026 \\ & & & & & & & & \\ 5 & 1 & 5 & \leftarrow & 4 & 1 & 4 & 3593.0607 & -0.0013 \\ 5 & 0 & 5 & \leftarrow & 4 & 0 & 4 & 3661.5802 & -0.0006 \\ 5 & 2 & 4 & \leftarrow & 4 & 2 & 3 & 3711.6807 & -0.0020 \\ 5 & 3 & 3 & \leftarrow & 4 & 3 & 2 & 3727.5469 & 0.0036 \\ 5 & 3 & 2 & \leftarrow & 4 & 3 & 1 & 3730.1712 & -0.0035 \\ 5 & 2 & 3 & \leftarrow & 4 & 2 & 2 & 3768.4663 & 0.0049 \\ 5 & 1 & 4 & \leftarrow & 4 & 1 & 3 & 3817.1533 & 0.0010 \\ & & & & & & & & \\ 6 & 0 & 6 & \leftarrow & 5 & 0 & 5 & 4368.3915 & -0.0026 \\ 6 & 2 & 5 & \leftarrow & 5 & 2 & 4 & 4448.4893 & -0.0003 \\ 6 & 3 & 4 & \leftarrow & 5 & 3 & 3 & 4475.3801 & -0.0033 \\ 6 & 3 & 3 & \leftarrow & 5 & 3 & 2 & 4482.3201 & -0.0017 \\ 6 & 2 & 4 & \leftarrow & 5 & 2 & 3 & 4541.9830 & -0.0050 \\ 6 & 1 & 5 & \leftarrow & 5 & 1 & 4 & 4570.2548 & 0.0002 \\ & & & & & & & & \\ 7 & 1 & 7 & \leftarrow & 6 & 1 & 6 & 5014.5464 & -0.0008 \\ 7 & 0 & 7 & \leftarrow & 6 & 0 & 6 & 5067.9080 & 0.0036 \\ 7 & 2 & 6 & \leftarrow & 6 & 2 & 5 & 5182.3561 & 0.0000 \\ 7 & 4 & 4 & \leftarrow & 6 & 4 & 3 & 5220.5957 & 0.0024 \\ 7 & 4 & 3 & \leftarrow & 6 & 4 & 2 & 5221.1120 & -0.0015 \\ 7 & 3 & 5 & \leftarrow & 6 & 3 & 4 & 5223.5634 & -0.0078 \\ 7 & 3 & 4 & \leftarrow & 6 & 3 & 3 & 5238.8832 & -0.0037 \\ 7 & 1 & 6 & \leftarrow & 6 & 1 & 5 & 5316.6359 & 0.0011 \\ 7 & 2 & 5 & \leftarrow & 6 & 2 & 4 & 5319.6230 & -0.0001 \\ & & & & & & & & \\ 8 & 1 & 8 & \leftarrow & 7 & 1 & 7 & 5721.4954 & 0.0027 \\ 8 & 0 & 8 & \leftarrow & 7 & 0 & 7 & 5763.1788 & 0.0010 \\ 8 & 2 & 7 & \leftarrow & 7 & 2 & 6 & 5912.8952 & -0.0019 \\ 8 & 5 & 4 & \leftarrow & 7 & 5 & 3 & 5964.2219 & 0.0118 \\ 8 & 3 & 5 & \leftarrow & 7 & 3 & 4 & 6001.2682 & 0.0009 \\ 8 & 1 & 7 & \leftarrow & 7 & 1 & 6 & 6054.5040 & 0.0015\end{array}$




$\begin{array}{ccccccccc}8 & 2 & 6 & \leftarrow & 7 & 2 & 5 & 6097.6257 & 0.0061 \\ 9 & 0 & 9 & \leftarrow & 8 & 0 & 8 & 6456.8922 & 0.0005 \\ 9 & 2 & 8 & \leftarrow & 8 & 2 & 7 & 6639.8187 & 0.0043 \\ 9 & 6 & 4 & \leftarrow & 8 & 6 & 3 & 6708.1132 & -0.0110 \\ 9 & 3 & 7 & \leftarrow & 8 & 3 & 6 & 6718.6498 & 0.0018 \\ 9 & 4 & 6 & \leftarrow & 8 & 4 & 5 & 6720.6372 & -0.0077 \\ 9 & 4 & 5 & \leftarrow & 8 & 4 & 4 & 6724.0041 & -0.0022 \\ 9 & 1 & 8 & \leftarrow & 8 & 1 & 7 & 6782.2292 & -0.0022 \\ & & & & & & & & \\ 10 & 1 & 10 & \leftarrow & 9 & 1 & 9 & 7129.2323 & 0.0040 \\ 10 & 0 & 10 & \leftarrow & 9 & 0 & 9 & 7150.6972 & 0.0045 \\ 10 & 8 & 2 & \leftarrow & 9 & 8 & 1 & 7449.8347 & 0.0062 \\ 10 & 3 & 8 & \leftarrow & 9 & 3 & 7 & 7464.1231 & -0.0010 \\ 10 & 4 & 6 & \leftarrow & 9 & 4 & 5 & 7479.4341 & -0.0030 \\ 10 & 1 & 9 & \leftarrow & 9 & 1 & 8 & 7498.9049 & 0.0012 \\ 10 & 2 & 8 & \leftarrow & 9 & 2 & 7 & 7641.5840 & -0.0105\end{array}$

c-type transitions:

\begin{tabular}{ccccccccc}
4 & 3 & 2 & $\leftarrow$ & 3 & 2 & 1 & 5512.4906 & -0.0050 \\
5 & 1 & 4 & $\leftarrow$ & 4 & 0 & 4 & 4589.0221 & -0.0104 \\
4 & 2 & 3 & $\leftarrow$ & 3 & 1 & 3 & 4637.4070 & 0.0014 \\
3 & 3 & 0 & $\leftarrow$ & 2 & 2 & 0 & 4775.2698 & 0.0086 \\
5 & 2 & 3 & $\leftarrow$ & 4 & 1 & 3 & 5119.4604 & 0.0065 \\
5 & 2 & 4 & $\leftarrow$ & 4 & 1 & 4 & 5470.7378 & 0.0025 \\
6 & 1 & 5 & $\leftarrow$ & 5 & 0 & 5 & 5497.7146 & 0.0083 \\
4 & 3 & 1 & $\leftarrow$ & 3 & 2 & 1 & 5513.3774 & -0.0011 \\
4 & 3 & 2 & $\leftarrow$ & 3 & 2 & 2 & 5527.5287 & -0.0024 \\
6 & 2 & 4 & $\leftarrow$ & 5 & 1 & 4 & 5844.2981 & 0.0085 \\
5 & 3 & 2 & $\leftarrow$ & 4 & 2 & 2 & 6241.7051 & -0.0036 \\
5 & 3 & 3 & $\leftarrow$ & 4 & 2 & 3 & 6282.6893 & -0.0058 \\
7 & 1 & 6 & $\leftarrow$ & 6 & 0 & 6 & 6445.9532 & 0.0065 \\
7 & 2 & 5 & $\leftarrow$ & 6 & 1 & 5 & 6593.6626 & 0.0043 \\
6 & 3 & 3 & $\leftarrow$ & 5 & 2 & 3 & 6955.5673 & -0.0019 \\
6 & 3 & 4 & $\leftarrow$ & 5 & 2 & 4 & 7046.3953 & -0.0006 \\
8 & 2 & 6 & $\leftarrow$ & 7 & 1 & 6 & 7374.6423 & -0.0007 \\
7 & 3 & 4 & $\leftarrow$ & 6 & 2 & 4 & 7652.4664 & -0.0017 \\
& & & & & & & & \\
& & & & & & & RMS & $4.537 \mathrm{kHz}$ \\
\hline
\end{tabular}


Table S27: Measured rotational transitions $\left(\nu_{\mathrm{obs}}\right)$ for the $3 \mathrm{w}-{ }^{18} \mathrm{O} 3$ complex of camphor with three water molecules, and the residuals $\left(\nu_{\mathrm{obs}}-\nu_{\text {calc }}\right)$ for the fit reported in Table S22 (see Figure S5 for oxygen atom numbering).

\begin{tabular}{ccccccccc}
\hline$J^{\prime}$ & $K_{a}^{\prime}$ & $K_{c}^{\prime}$ & $\leftarrow$ & $J^{\prime \prime}$ & $K_{a}^{\prime \prime}$ & $K_{c}^{\prime \prime}$ & $\nu_{\mathrm{obs}}(\mathrm{MHz})$ & $\nu_{\mathrm{obs}}-\nu_{\text {calc }}$ \\
\hline
\end{tabular}

$a$-type transitions:

$\begin{array}{ccccccccc}3 & 1 & 3 & \leftarrow & 2 & 1 & 2 & 2148.2449 & -0.0008 \\ 3 & 0 & 3 & \leftarrow & 2 & 0 & 2 & 2203.2516 & -0.0058 \\ 3 & 1 & 2 & \leftarrow & 2 & 1 & 1 & 2275.6261 & 0.0004 \\ & & & & & & & & \\ 4 & 1 & 4 & \leftarrow & 3 & 1 & 3 & 2861.5163 & -0.0003 \\ 4 & 0 & 4 & \leftarrow & 3 & 0 & 3 & 2926.0562 & 0.0033 \\ 4 & 2 & 3 & \leftarrow & 3 & 2 & 2 & 2949.3857 & -0.0026 \\ 4 & 2 & 2 & \leftarrow & 3 & 2 & 1 & 2974.7304 & 0.0003 \\ 4 & 1 & 3 & \leftarrow & 3 & 1 & 2 & 3030.8972 & 0.0009 \\ & & & & & & & & \\ 5 & 1 & 5 & \leftarrow & 4 & 1 & 4 & 3572.6588 & 0.0016 \\ 5 & 3 & 3 & \leftarrow & 4 & 3 & 2 & 3697.1630 & 0.0024 \\ 5 & 3 & 2 & \leftarrow & 4 & 3 & 1 & 3699.2261 & 0.0035 \\ 5 & 2 & 3 & \leftarrow & 4 & 2 & 2 & 3732.5936 & 0.0000 \\ 5 & 1 & 4 & \leftarrow & 4 & 1 & 3 & 3783.0598 & -0.0002 \\ & & & & & & & & \\ 6 & 1 & 6 & \leftarrow & 5 & 1 & 5 & 4281.4574 & 0.0011 \\ 6 & 0 & 6 & \leftarrow & 5 & 0 & 5 & 4345.3866 & 0.0059 \\ 6 & 2 & 5 & \leftarrow & 5 & 2 & 4 & 4415.4380 & 0.0031 \\ 6 & 3 & 4 & \leftarrow & 5 & 3 & 3 & 4438.7068 & 0.0000 \\ 6 & 3 & 3 & \leftarrow & 5 & 3 & 2 & 4444.1543 & 0.0005 \\ 6 & 2 & 4 & \leftarrow & 5 & 2 & 3 & 4496.9937 & 0.0009 \\ 6 & 1 & 5 & \leftarrow & 5 & 1 & 4 & 4530.9684 & 0.0021 \\ & & & & & & & & \\ 7 & 1 & 7 & \leftarrow & 6 & 1 & 6 & 4987.8807 & 0.0109 \\ 7 & 2 & 6 & \leftarrow & 6 & 2 & 5 & 5144.8482 & -0.0030 \\ 7 & 3 & 5 & \leftarrow & 6 & 3 & 4 & 5180.6611 & -0.0038 \\ 7 & 3 & 4 & \leftarrow & 6 & 3 & 3 & 5192.7229 & -0.0052 \\ 7 & 2 & 5 & \leftarrow & 6 & 2 & 4 & 5265.8584 & -0.0034 \\ 7 & 1 & 6 & \leftarrow & 6 & 1 & 5 & 5273.2507 & -0.0016 \\ & & & & & & & & \\ 8 & 1 & 8 & \leftarrow & 7 & 1 & 7 & 5692.0103 & 0.0048 \\ 8 & 0 & 8 & \leftarrow & 7 & 0 & 7 & 5736.9059 & -0.0164 \\ 8 & 2 & 7 & \leftarrow & 7 & 2 & 6 & 5871.3864 & -0.0002 \\ 8 & 5 & 4 & \leftarrow & 7 & 5 & 3 & 5915.4284 & 0.0019 \\ 8 & 4 & 5 & \leftarrow & 7 & 4 & 4 & 5920.4937 & 0.0034 \\ 8 & 4 & 4 & \leftarrow & 7 & 4 & 3 & 5921.5018 & -0.0055 \\ 8 & 3 & 6 & \leftarrow & 7 & 3 & 5 & 5922.5962 & -0.0025 \\ 8 & 3 & 5 & \leftarrow & 7 & 3 & 4 & 5946.1245 & -0.0040 \\ 8 & 1 & 7 & \leftarrow & 7 & 1 & 6 & 6008.3953 & -0.0044 \\ 8 & 2 & 6 & \leftarrow & 7 & 2 & 5 & 6036.0919 & -0.0044\end{array}$




$\begin{array}{ccccccccc}9 & 1 & 9 & \leftarrow & 8 & 1 & 8 & 6394.0943 & 0.0050 \\ 9 & 0 & 9 & \leftarrow & 8 & 0 & 8 & 6428.2215 & -0.0072 \\ 9 & 2 & 8 & \leftarrow & 8 & 2 & 7 & 6594.7567 & -0.0019 \\ 9 & 3 & 7 & \leftarrow & 8 & 3 & 6 & 6663.9482 & -0.0082 \\ 9 & 4 & 6 & \leftarrow & 8 & 4 & 5 & 6664.3533 & -0.0129 \\ 9 & 3 & 6 & \leftarrow & 8 & 3 & 5 & 6705.4856 & -0.0097 \\ 9 & 1 & 8 & \leftarrow & 8 & 1 & 7 & 6734.9253 & 0.0017 \\ 10 & 1 & 10 & \leftarrow & 9 & 1 & 9 & 7094.4264 & 0.0069 \\ 10 & 0 & 10 & \leftarrow & 9 & 0 & 9 & 7119.1537 & 0.0021 \\ 10 & 2 & 9 & \leftarrow & 9 & 2 & 8 & 7314.7710 & 0.0000 \\ 10 & 4 & 7 & \leftarrow & 5 & 4 & 6 & 7409.2552 & 0.0343 \\ 10 & 2 & 8 & \leftarrow & 5 & 2 & 7 & 7568.6286 & -0.0065\end{array}$

c-type transitions:

\begin{tabular}{ccccccccc}
4 & 1 & 3 & $\leftarrow$ & 3 & 0 & 3 & 3691.7061 & 0.0050 \\
3 & 2 & 1 & $\leftarrow$ & 2 & 1 & 1 & 3729.0459 & 0.0146 \\
3 & 2 & 2 & $\leftarrow$ & 2 & 1 & 2 & 3843.6190 & -0.0017 \\
4 & 2 & 3 & $\leftarrow$ & 3 & 1 & 3 & 4644.7721 & 0.0087 \\
3 & 3 & 0 & $\leftarrow$ & 2 & 2 & 0 & 4823.1783 & -0.0036 \\
3 & 3 & 1 & $\leftarrow$ & 2 & 2 & 1 & 4825.6766 & 0.0030 \\
5 & 2 & 3 & $\leftarrow$ & 4 & 1 & 3 & 5129.8402 & 0.0073 \\
6 & 1 & 5 & $\leftarrow$ & 5 & 0 & 5 & 5439.5809 & 0.0022 \\
5 & 2 & 4 & $\leftarrow$ & 4 & 1 & 4 & 5466.7391 & 0.0081 \\
4 & 3 & 1 & $\leftarrow$ & 3 & 2 & 1 & 5556.2214 & -0.0032 \\
4 & 3 & 2 & $\leftarrow$ & 3 & 2 & 2 & 5568.4197 & -0.0033 \\
6 & 2 & 4 & $\leftarrow$ & 5 & 1 & 4 & 5843.7746 & 0.0089 \\
5 & 3 & 2 & $\leftarrow$ & 4 & 2 & 2 & 6280.7127 & -0.0043 \\
6 & 2 & 5 & $\leftarrow$ & 5 & 1 & 5 & 6309.5151 & 0.0064 \\
5 & 3 & 3 & $\leftarrow$ & 4 & 2 & 3 & 6316.1892 & -0.0061 \\
7 & 2 & 5 & $\leftarrow$ & 6 & 1 & 5 & 6578.6658 & 0.0045 \\
6 & 3 & 3 & $\leftarrow$ & 5 & 2 & 3 & 6992.2665 & -0.0108 \\
7 & 2 & 6 & $\leftarrow$ & 6 & 1 & 6 & 7172.9041 & 0.0005 \\
8 & 2 & 6 & $\leftarrow$ & 7 & 1 & 6 & 7341.4972 & -0.0079 \\
7 & 3 & 4 & $\leftarrow$ & 6 & 2 & 4 & 7688.0102 & -0.0024 \\
& & & & & & & & \\
& & & & & & & $\mathrm{RMS}$ & $6.952 \mathrm{kHz}$ \\
\hline
\end{tabular}


Table S28: Measured rotational transitions ( $\left.\nu_{\text {obs }}\right)$ for the $3 \mathrm{w}^{-18} \mathrm{O} 1^{18} \mathrm{O} 2$ complex of camphor with three water molecules, and the residuals $\left(\nu_{\text {obs }}-\nu_{\text {calc }}\right)$ for the fit reported in Table S22 (see Figure S5 for oxygen atom numbering).

\begin{tabular}{ccccccccc}
\hline$J^{\prime}$ & $K_{a}^{\prime}$ & $K_{c}^{\prime}$ & $\leftarrow$ & $J^{\prime \prime}$ & $K_{a}^{\prime \prime}$ & $K_{c}^{\prime \prime}$ & $\nu_{\mathrm{obs}}(\mathrm{MHz})$ & $\nu_{\mathrm{obs}}-\nu_{\text {calc }}$ \\
\hline
\end{tabular}

$a$-type transitions:

$\begin{array}{ccccccccc}3 & 1 & 3 & \leftarrow & 2 & 1 & 2 & 2134.1037 & -0.0068 \\ 3 & 0 & 3 & \leftarrow & 2 & 0 & 2 & 2193.0212 & -0.0002 \\ 3 & 1 & 2 & \leftarrow & 2 & 1 & 1 & 2273.8050 & 0.0036 \\ & & & & & & & & \\ 4 & 1 & 4 & \leftarrow & 3 & 1 & 3 & 2841.9741 & -0.0001 \\ 4 & 0 & 4 & \leftarrow & 3 & 0 & 3 & 2909.5302 & -0.0048 \\ 4 & 2 & 3 & \leftarrow & 3 & 2 & 2 & 2938.7858 & -0.0012 \\ 4 & 2 & 2 & \leftarrow & 3 & 2 & 1 & 2970.5724 & -0.0047 \\ & & & & & & & & \\ 5 & 1 & 5 & \leftarrow & 4 & 1 & 4 & 3547.2330 & -0.0007 \\ 5 & 0 & 5 & \leftarrow & 4 & 0 & 4 & 3615.5651 & -0.0018 \\ 5 & 2 & 4 & \leftarrow & 4 & 2 & 3 & 3669.3835 & -0.0010 \\ 5 & 3 & 3 & \leftarrow & 4 & 3 & 2 & 3686.4713 & -0.0013 \\ 5 & 3 & 2 & \leftarrow & 4 & 3 & 1 & 3689.4645 & -0.0022 \\ 5 & 2 & 3 & \leftarrow & 4 & 2 & 2 & 3730.4205 & -0.0022 \\ 5 & 1 & 4 & \leftarrow & 4 & 1 & 3 & 3777.2967 & -0.0008 \\ & & & & & & & & \\ 6 & 1 & 6 & \leftarrow & 5 & 1 & 5 & 4249.6925 & 0.0002 \\ 6 & 0 & 6 & \leftarrow & 5 & 0 & 5 & 4311.7794 & -0.0024 \\ 6 & 2 & 5 & \leftarrow & 5 & 2 & 4 & 4397.2840 & -0.0023 \\ 6 & 3 & 4 & \leftarrow & 5 & 3 & 3 & 4426.2013 & 0.0015 \\ 6 & 3 & 3 & \leftarrow & 5 & 3 & 2 & 4434.0867 & 0.0026 \\ 6 & 2 & 4 & \leftarrow & 5 & 2 & 3 & 4497.2711 & 0.0012 \\ 6 & 1 & 5 & \leftarrow & 5 & 1 & 4 & 4521.4764 & 0.0005 \\ & & & & & & & & \\ 7 & 1 & 7 & \leftarrow & 6 & 1 & 6 & 4949.3761 & 0.0005 \\ 7 & 0 & 7 & \leftarrow & 6 & 0 & 6 & 5000.7752 & 0.0025 \\ 7 & 2 & 6 & \leftarrow & 6 & 2 & 5 & 5122.0184 & -0.0010 \\ 7 & 5 & 2 & \leftarrow & 6 & 5 & 1 & 5158.9455 & -0.0043 \\ 7 & 4 & 4 & \leftarrow & 6 & 4 & 3 & 5163.3379 & 0.0022 \\ 7 & 3 & 5 & \leftarrow & 6 & 3 & 4 & 5166.2188 & 0.0017 \\ 7 & 3 & 4 & \leftarrow & 6 & 3 & 3 & 5183.5818 & -0.0010 \\ 7 & 1 & 6 & \leftarrow & 6 & 1 & 5 & 5258.2785 & -0.0028 \\ 7 & 2 & 5 & \leftarrow & 6 & 2 & 4 & 5267.8674 & -0.0032 \\ & & & & & & & & \\ 8 & 0 & 8 & \leftarrow & 7 & 0 & 7 & 5685.8630 & -0.0002 \\ 8 & 2 & 7 & \leftarrow & 7 & 2 & 6 & 5843.1812 & 0.0005 \\ 8 & 4 & 5 & \leftarrow & 7 & 4 & 4 & 5904.8651 & -0.0004 \\ 8 & 3 & 6 & \leftarrow & 7 & 3 & 5 & 5905.9020 & 0.0040 \\ 8 & 3 & 5 & \leftarrow & 7 & 3 & 4 & 5939.4669 & -0.0055 \\ 8 & 1 & 7 & \leftarrow & 7 & 1 & 6 & 5985.7746 & -0.0038 \\ & & & & & & & & \end{array}$




$\begin{array}{ccccccccc}8 & 2 & 6 & \leftarrow & 7 & 2 & 5 & 6038.1452 & -0.0008 \\ 9 & 1 & 9 & \leftarrow & 8 & 1 & 8 & 6341.4034 & -0.0002 \\ 9 & 0 & 9 & \leftarrow & 8 & 0 & 8 & 6369.7599 & 0.0021 \\ 9 & 2 & 8 & \leftarrow & 8 & 2 & 7 & 6560.4674 & -0.0019 \\ 9 & 6 & 3 & \leftarrow & 8 & 6 & 2 & 6634.2087 & 0.0038 \\ 9 & 3 & 7 & \leftarrow & 8 & 3 & 6 & 6644.4953 & 0.0037 \\ 9 & 1 & 8 & \leftarrow & 8 & 1 & 7 & 6702.3031 & -0.0036 \\ 9 & 3 & 6 & \leftarrow & 8 & 3 & 5 & 6702.9608 & -0.0098 \\ & & & & & & & & \\ 10 & 1 & 10 & \leftarrow & 9 & 1 & 9 & 7034.5041 & 0.0066 \\ 10 & 0 & 10 & \leftarrow & 9 & 0 & 9 & 7053.9929 & 0.0044 \\ 10 & 2 & 9 & \leftarrow & 9 & 2 & 8 & 7273.7186 & 0.0005 \\ 10 & 3 & 8 & \leftarrow & 9 & 3 & 7 & 7381.1964 & -0.0017 \\ 10 & 1 & 9 & \leftarrow & 9 & 1 & 8 & 7407.1406 & 0.0046 \\ 10 & 3 & 7 & \leftarrow & 9 & 3 & 6 & 7474.3284 & -0.0078 \\ 10 & 2 & 8 & \leftarrow & 9 & 2 & 7 & 7564.2202 & -0.0073\end{array}$

c-type transitions:

\begin{tabular}{ccccccccc}
2 & 2 & 0 & $\leftarrow$ & 1 & 1 & 0 & 2942.0352 & 0.0042 \\
3 & 2 & 1 & $\leftarrow$ & 2 & 1 & 1 & 3643.7488 & 0.0112 \\
4 & 2 & 2 & $\leftarrow$ & 3 & 1 & 2 & 4340.5235 & 0.0102 \\
3 & 3 & 0 & $\leftarrow$ & 2 & 2 & 0 & 4690.5110 & -0.0025 \\
3 & 3 & 1 & $\leftarrow$ & 2 & 2 & 1 & 4693.6343 & -0.0076 \\
5 & 2 & 3 & $\leftarrow$ & 4 & 1 & 3 & 5043.3871 & 0.0103 \\
5 & 2 & 4 & $\leftarrow$ & 4 & 1 & 4 & 5399.4154 & 0.0121 \\
4 & 3 & 1 & $\leftarrow$ & 3 & 2 & 1 & 5419.8557 & -0.0025 \\
4 & 3 & 2 & $\leftarrow$ & 3 & 2 & 2 & 5435.0999 & -0.0089 \\
6 & 2 & 4 & $\leftarrow$ & 5 & 1 & 4 & 5763.3548 & 0.0056 \\
5 & 3 & 2 & $\leftarrow$ & 4 & 2 & 2 & 6138.7373 & -0.0106 \\
5 & 3 & 3 & $\leftarrow$ & 4 & 2 & 3 & 6182.7942 & -0.0002 \\
6 & 2 & 5 & $\leftarrow$ & 5 & 1 & 5 & 6249.4659 & 0.0100 \\
7 & 1 & 6 & $\leftarrow$ & 6 & 0 & 6 & 6398.8365 & 0.0066 \\
7 & 2 & 5 & $\leftarrow$ & 6 & 1 & 5 & 6509.7523 & 0.0084 \\
6 & 3 & 3 & $\leftarrow$ & 5 & 2 & 3 & 6842.4078 & -0.0012 \\
8 & 2 & 6 & $\leftarrow$ & 7 & 1 & 6 & 7289.6174 & 0.0087 \\
7 & 3 & 5 & $\leftarrow$ & 6 & 2 & 5 & 7708.5382 & -0.0020 \\
4 & 3 & 1 & $\leftarrow$ & 3 & 2 & 2 & 5436.1046 & -0.0095 \\
6 & 2 & 4 & $\leftarrow$ & 5 & 1 & 5 & 6458.5190 & -0.0045 \\
& & & & & & & & \\
& & & & & & & $\mathrm{RMS}$ & $5.075 \mathrm{kHz}$ \\
\hline
\end{tabular}


Table S29: Measured rotational transitions $\left(\nu_{\text {obs }}\right)$ for the $3 \mathrm{w}^{-18} \mathrm{O} 1^{18} \mathrm{O} 3$ complex of camphor with three water molecules, and the residuals $\left(\nu_{\text {obs }}-\nu_{\text {calc }}\right)$ for the fit reported in Table S22 (see Figure S5 for oxygen atom numbering).

\begin{tabular}{ccccccccc}
\hline$J^{\prime}$ & $K_{a}^{\prime}$ & $K_{c}^{\prime}$ & $\leftarrow$ & $J^{\prime \prime}$ & $K_{a}^{\prime \prime}$ & $K_{c}^{\prime \prime}$ & $\nu_{\mathrm{obs}}(\mathrm{MHz})$ & $\nu_{\mathrm{obs}}-\nu_{\text {calc }}$ \\
\hline
\end{tabular}

$a$-type transitions:

$\begin{array}{ccccccccc}3 & 1 & 3 & \leftarrow & 2 & 1 & 2 & 2121.6517 & -0.0045 \\ 3 & 0 & 3 & \leftarrow & 2 & 0 & 2 & 2177.6363 & -0.0022 \\ & & & & & & & & \\ 4 & 1 & 4 & \leftarrow & 3 & 1 & 3 & 2825.8635 & -0.0028 \\ 4 & 0 & 4 & \leftarrow & 3 & 0 & 3 & 2891.0753 & -0.0008 \\ 4 & 2 & 3 & \leftarrow & 3 & 2 & 2 & 2916.0915 & -0.0021 \\ 4 & 2 & 2 & \leftarrow & 3 & 2 & 1 & 2943.2735 & 0.0044 \\ 4 & 1 & 3 & \leftarrow & 3 & 1 & 2 & 2999.4818 & -0.0015 \\ & & & & & & & & \\ 5 & 1 & 5 & \leftarrow & 4 & 1 & 4 & 3527.8106 & -0.0001 \\ 5 & 0 & 5 & \leftarrow & 4 & 0 & 4 & 3595.2583 & -0.0010 \\ 5 & 2 & 4 & \leftarrow & 4 & 2 & 3 & 3641.6240 & -0.0014 \\ 5 & 3 & 2 & \leftarrow & 4 & 3 & 1 & 3658.5913 & 0.0002 \\ 5 & 2 & 3 & \leftarrow & 4 & 2 & 2 & 3694.1383 & -0.0005 \\ 5 & 1 & 4 & \leftarrow & 4 & 1 & 3 & 3743.3428 & -0.0017 \\ & & & & & & & & \\ 6 & 1 & 6 & \leftarrow & 5 & 1 & 5 & 4227.2806 & -0.0013 \\ 6 & 0 & 6 & \leftarrow & 5 & 0 & 5 & 4290.3674 & -0.0005 \\ 6 & 3 & 4 & \leftarrow & 5 & 3 & 3 & 4389.7319 & 0.0005 \\ 6 & 3 & 3 & \leftarrow & 5 & 3 & 2 & 4395.8559 & -0.0008 \\ 6 & 2 & 4 & \leftarrow & 5 & 2 & 3 & 4451.6920 & -0.0018 \\ 6 & 1 & 5 & \leftarrow & 5 & 1 & 4 & 4482.5800 & -0.0011 \\ & & & & & & & & \\ 7 & 1 & 7 & \leftarrow & 6 & 1 & 6 & 4924.2553 & 0.0000 \\ 7 & 0 & 7 & \leftarrow & 6 & 0 & 6 & 4978.2720 & 0.0014 \\ 7 & 2 & 6 & \leftarrow & 6 & 2 & 5 & 5085.3739 & 0.0027 \\ 7 & 5 & 3 & \leftarrow & 6 & 5 & 2 & 5116.8193 & -0.0026 \\ 7 & 5 & 2 & \leftarrow & 6 & 5 & 1 & 5116.8193 & -0.0087 \\ 7 & 4 & 4 & \leftarrow & 6 & 4 & 3 & 5120.5942 & 0.0015 \\ 7 & 4 & 3 & \leftarrow & 6 & 4 & 2 & 5121.0292 & -0.0029 \\ 7 & 3 & 5 & \leftarrow & 6 & 3 & 4 & 5123.5699 & -0.0020 \\ 7 & 3 & 4 & \leftarrow & 6 & 3 & 3 & 5137.1167 & 0.0010 \\ 7 & 2 & 5 & \leftarrow & 6 & 2 & 4 & 5213.5233 & 0.0011 \\ 7 & 1 & 6 & \leftarrow & 6 & 1 & 5 & 5215.7000 & -0.0022 \\ & & & & & & & & \\ 8 & 1 & 8 & \leftarrow & 7 & 1 & 7 & 5618.8741 & 0.0049 \\ 8 & 0 & 8 & \leftarrow & 7 & 0 & 7 & 5661.7613 & 0.0018 \\ 8 & 2 & 7 & \leftarrow & 7 & 2 & 6 & 5802.8067 & -0.0017 \\ 8 & 6 & 3 & \leftarrow & 7 & 6 & 2 & 5846.9906 & -0.0023 \\ 8 & 5 & 4 & \leftarrow & 7 & 5 & 3 & 5850.0865 & 0.0110 \\ 8 & 4 & 5 & \leftarrow & 7 & 4 & 4 & 5855.4847 & 0.0025\end{array}$




$\begin{array}{ccccccccc}8 & 4 & 4 & \leftarrow & 7 & 4 & 3 & 5856.6768 & -0.0044 \\ 8 & 3 & 6 & \leftarrow & 7 & 3 & 5 & 5857.3014 & 0.0001 \\ 8 & 3 & 5 & \leftarrow & 7 & 3 & 4 & 5883.6464 & -0.0026 \\ 8 & 1 & 7 & \leftarrow & 7 & 1 & 6 & 5941.0639 & -0.0009 \\ 8 & 2 & 6 & \leftarrow & 7 & 2 & 5 & 5976.2116 & -0.0060 \\ & & & & & & & & \\ 9 & 1 & 9 & \leftarrow & 8 & 1 & 8 & 6311.3883 & 0.0040 \\ 9 & 0 & 9 & \leftarrow & 8 & 0 & 8 & 6343.4207 & 0.0018 \\ 9 & 2 & 8 & \leftarrow & 8 & 2 & 7 & 6516.8831 & 0.0047 \\ 9 & 6 & 4 & \leftarrow & 8 & 6 & 3 & 6579.8489 & 0.0026 \\ 9 & 3 & 7 & \leftarrow & 8 & 3 & 6 & 6590.3103 & 0.0007 \\ 9 & 4 & 6 & \leftarrow & 8 & 4 & 5 & 6591.4666 & -0.0028 \\ 9 & 3 & 6 & \leftarrow & 8 & 3 & 5 & 6636.6312 & -0.0051 \\ 9 & 1 & 8 & \leftarrow & 8 & 1 & 7 & 6657.1121 & -0.0055 \\ 9 & 2 & 7 & \leftarrow & 8 & 2 & 6 & 6736.4675 & -0.0037 \\ & & & & & & & & \\ 10 & 1 & 10 & \leftarrow & 9 & 1 & 9 & 7002.1331 & 0.0021 \\ 10 & 0 & 10 & \leftarrow & 9 & 0 & 9 & 7024.9504 & 0.0066 \\ 10 & 2 & 9 & \leftarrow & 9 & 2 & 8 & 7227.3854 & -0.0061 \\ 10 & 3 & 8 & \leftarrow & 9 & 3 & 7 & 7321.9332 & 0.0079 \\ 10 & 1 & 9 & \leftarrow & 9 & 1 & 8 & 7362.8520 & 0.0000 \\ 10 & 2 & 8 & \leftarrow & 9 & 2 & 7 & 7491.6981 & -0.0103\end{array}$

$c$-type transitions:

\begin{tabular}{ccccccccc}
6 & 3 & 4 & $\leftarrow$ & 5 & 2 & 3 & 6873.7503 & 0.0102 \\
7 & 3 & 5 & $\leftarrow$ & 6 & 2 & 4 & 7545.6084 & -0.0098 \\
3 & 1 & 2 & $\leftarrow$ & 2 & 0 & 2 & 2831.1577 & 0.0034 \\
5 & 1 & 4 & $\leftarrow$ & 4 & 0 & 4 & 4505.2684 & 0.0009 \\
4 & 2 & 3 & $\leftarrow$ & $\leftarrow$ & 1 & 3 & 4580.7723 & 0.0062 \\
3 & 3 & 0 & $\leftarrow$ & 2 & 2 & 0 & 4741.5065 & 0.0010 \\
6 & 1 & 5 & $\leftarrow$ & 5 & 0 & 5 & 5392.5931 & 0.0039 \\
5 & 2 & 4 & $\leftarrow$ & 4 & 1 & 4 & 5396.5325 & 0.0073 \\
4 & 3 & 1 & $\leftarrow$ & 3 & 2 & 1 & 5465.9206 & -0.0042 \\
4 & 3 & 2 & $\leftarrow$ & 3 & 2 & 2 & 5478.9815 & -0.0094 \\
6 & 2 & 4 & $\leftarrow$ & 5 & 1 & 4 & 5763.4832 & 0.0066 \\
5 & 3 & 2 & $\leftarrow$ & 4 & 2 & 2 & 6181.2432 & -0.0035 \\
6 & 2 & 5 & $\leftarrow$ & 5 & 1 & 5 & 6233.5793 & 0.0091 \\
7 & 2 & 5 & $\leftarrow$ & 6 & 1 & 5 & 6494.4209 & 0.0033 \\
6 & 3 & 3 & $\leftarrow$ & 5 & 2 & 3 & 6882.9653 & 0.0005 \\
6 & 3 & 4 & $\leftarrow$ & 5 & 2 & 4 & 6967.2685 & -0.0048 \\
8 & 2 & 6 & $\leftarrow$ & 7 & 1 & 6 & 7254.9374 & 0.0045 \\
7 & 3 & 4 & $\leftarrow$ & 6 & 2 & 4 & 7568.3835 & -0.0029 \\
& & & & & & & & \\
& & & & & & & RMS & $4.365 \mathrm{kHz}$ \\
\hline
\end{tabular}


Table S30: Measured rotational transitions ( $\left.\nu_{\text {obs }}\right)$ for the $3 \mathrm{w}^{18}{ }^{18} \mathrm{O}{ }^{18} \mathrm{O} 3$ complex of camphor with three water molecules, and the residuals $\left(\nu_{\text {obs }}-\nu_{\text {calc }}\right)$ for the fit reported in Table S22 (see Figure S5 for oxygen atom numbering).

\begin{tabular}{ccccccccc}
\hline$J^{\prime}$ & $K_{a}^{\prime}$ & $K_{c}^{\prime}$ & $\leftarrow$ & $J^{\prime \prime}$ & $K_{a}^{\prime \prime}$ & $K_{c}^{\prime \prime}$ & $\nu_{\mathrm{obs}}(\mathrm{MHz})$ & $\nu_{\mathrm{obs}}-\nu_{\text {calc }}$ \\
\hline
\end{tabular}

$a$-type transitions:

$\begin{array}{ccccccccc}3 & 0 & 3 & \leftarrow & 2 & 0 & 2 & 2164.7186 & -0.0025 \\ 3 & 1 & 2 & \leftarrow & 2 & 1 & 1 & 2238.3751 & 0.0024 \\ & & & & & & & & \\ 4 & 1 & 4 & \leftarrow & 3 & 1 & 3 & 2809.2662 & -0.0017 \\ 4 & 0 & 4 & \leftarrow & 3 & 0 & 3 & 2874.2114 & -0.0012 \\ 4 & 2 & 3 & \leftarrow & 3 & 2 & 2 & 2898.4959 & 0.0066 \\ 4 & 1 & 3 & \leftarrow & 3 & 1 & 2 & 2981.0810 & -0.0021 \\ & & & & & & & & \\ 5 & 1 & 5 & \leftarrow & 4 & 1 & 4 & 3507.1826 & -0.0049 \\ 5 & 0 & 5 & \leftarrow & 4 & 0 & 4 & 3574.6640 & -0.0040 \\ 5 & 2 & 4 & \leftarrow & 4 & 2 & 3 & 3619.7282 & 0.0018 \\ 5 & 3 & 3 & \leftarrow & 4 & 3 & 2 & 3633.9447 & 0.0008 \\ 5 & 3 & 2 & \leftarrow & 4 & 3 & 1 & 3636.1441 & -0.0045 \\ 5 & 2 & 3 & \leftarrow & 4 & 2 & 2 & 3670.7462 & 0.0012 \\ 5 & 1 & 4 & \leftarrow & 4 & 1 & 3 & 3720.5428 & -0.0005 \\ & & & & & & & & \\ 6 & 1 & 6 & \leftarrow & 5 & 1 & 5 & 4202.6884 & -0.0030 \\ 6 & 0 & 6 & \leftarrow & 5 & 0 & 5 & 4266.1902 & 0.0082 \\ 6 & 2 & 5 & \leftarrow & 5 & 2 & 4 & 4338.7285 & -0.0023 \\ 6 & 3 & 4 & \leftarrow & 5 & 3 & 3 & 4362.8980 & -0.0024 \\ 6 & 3 & 3 & \leftarrow & 5 & 3 & 2 & 4368.7161 & -0.0056 \\ 6 & 2 & 4 & \leftarrow & 5 & 2 & 3 & 4423.2575 & -0.0008 \\ 6 & 1 & 5 & \leftarrow & 5 & 1 & 4 & 4455.5431 & 0.0011 \\ & & & & & & & & \\ 7 & 1 & 7 & \leftarrow & 6 & 1 & 6 & 4895.7480 & 0.0018 \\ 7 & 0 & 7 & \leftarrow & 6 & 0 & 6 & 4950.4852 & -0.0039 \\ 7 & 2 & 6 & \leftarrow & 6 & 2 & 5 & 5055.1065 & 0.0063 \\ 7 & 4 & 4 & \leftarrow & 6 & 4 & 3 & 5089.2559 & -0.0001 \\ 7 & 4 & 3 & \leftarrow & 6 & 4 & 2 & 5089.6633 & -0.0017 \\ 7 & 3 & 5 & \leftarrow & 6 & 3 & 4 & 5092.2507 & -0.0014 \\ 7 & 3 & 4 & \leftarrow & 6 & 3 & 3 & 5105.1301 & -0.0027 \\ 7 & 2 & 5 & \leftarrow & 6 & 2 & 4 & 5180.1368 & -0.0033 \\ 7 & 1 & 6 & \leftarrow & 6 & 1 & 5 & 5184.6446 & -0.0002 \\ & & & & & & & & \\ 8 & 1 & 8 & \leftarrow & 7 & 1 & 7 & 5586.4728 & -0.0036 \\ 8 & 0 & 8 & \leftarrow & 7 & 0 & 7 & 5630.2752 & 0.0005 \\ 8 & 2 & 7 & \leftarrow & 7 & 2 & 6 & 5768.4775 & -0.0007 \\ 8 & 5 & 4 & \leftarrow & 7 & 5 & 3 & 5814.3237 & 0.0081 \\ 8 & 4 & 5 & \leftarrow & 7 & 4 & 4 & 5819.5795 & 0.0039 \\ 8 & 4 & 4 & \leftarrow & 7 & 4 & 3 & 5820.6917 & -0.0001 \\ 8 & 3 & 6 & \leftarrow & 7 & 3 & 5 & 5821.5311 & -0.0013\end{array}$




$\begin{array}{ccccccccc}8 & 3 & 5 & \leftarrow & 7 & 3 & 4 & 5846.6192 & -0.0012 \\ 8 & 1 & 7 & \leftarrow & 7 & 1 & 6 & 5906.2681 & 0.0046 \\ 8 & 2 & 6 & \leftarrow & 7 & 2 & 5 & 5938.1119 & -0.0030 \\ & & & & & & & & \\ 9 & 1 & 9 & \leftarrow & 8 & 1 & 8 & 6275.1299 & 0.0024 \\ 9 & 0 & 9 & \leftarrow & 8 & 0 & 8 & 6308.0941 & 0.0015 \\ 9 & 2 & 8 & \leftarrow & 8 & 2 & 7 & 6478.5795 & 0.0010 \\ 9 & 3 & 7 & \leftarrow & 8 & 3 & 6 & 6550.1529 & -0.0045 \\ 9 & 4 & 6 & \leftarrow & 8 & 4 & 5 & 6550.9698 & -0.0025 \\ 9 & 4 & 5 & \leftarrow & 8 & 4 & 4 & 6553.6169 & -0.0048 \\ 9 & 1 & 8 & \leftarrow & 8 & 1 & 7 & 6618.8718 & -0.0017 \\ 9 & 2 & 7 & \leftarrow & \leftarrow & 2 & 6 & 6693.9301 & -0.0055 \\ & & & & & & & & \\ 10 & 1 & 10 & \leftarrow & 9 & 1 & 9 & 6962.0269 & 0.0112 \\ 10 & 0 & 10 & \leftarrow & 9 & 0 & 9 & 6985.6659 & -0.0024 \\ 10 & 2 & 9 & \leftarrow & 5 & 2 & 8 & 7185.2093 & 0.0013 \\ 10 & 3 & 8 & \leftarrow & 5 & 3 & 7 & 7277.4791 & 0.0001 \\ 10 & 1 & 9 & \leftarrow & 9 & 1 & 8 & 7321.4315 & 0.0022 \\ 10 & 3 & 7 & \leftarrow & 5 & 3 & 6 & 7348.9811 & -0.0065 \\ 10 & 2 & 8 & \leftarrow & 9 & 2 & 7 & 7445.0339 & -0.0043\end{array}$

$c$-type transitions:

\begin{tabular}{ccccccccc}
4 & 3 & 2 & $\leftarrow$ & 3 & 2 & 1 & 5473.2695 & -0.0118 \\
7 & 3 & 5 & $\leftarrow$ & 6 & 2 & 5 & 7718.8627 & 0.0077 \\
7 & 3 & 4 & $\leftarrow$ & 6 & 2 & 4 & 7565.1542 & -0.0084 \\
8 & 2 & 6 & $\leftarrow$ & 7 & 1 & 6 & 7228.7662 & 0.0073 \\
6 & 3 & 4 & $\leftarrow$ & 5 & 2 & 4 & 6965.3365 & 0.0028 \\
6 & 3 & 3 & $\leftarrow$ & 5 & 2 & 3 & 6883.2853 & -0.0029 \\
7 & 2 & 5 & $\leftarrow$ & 6 & 1 & 5 & 6475.2991 & 0.0103 \\
5 & 3 & 3 & $\leftarrow$ & 4 & 2 & 3 & 6222.1477 & -0.0118 \\
5 & 3 & 2 & $\leftarrow$ & 4 & 2 & 2 & 6185.3131 & 0.0016 \\
6 & 2 & 4 & $\leftarrow$ & 5 & 1 & 4 & 5750.6966 & 0.0059 \\
4 & 3 & 1 & $\leftarrow$ & $\leftarrow$ & 2 & 1 & 5474.0185 & -0.0020 \\
5 & 2 & 4 & $\leftarrow$ & 4 & 1 & 4 & 5387.5599 & 0.0127 \\
6 & 1 & 5 & $\leftarrow$ & 5 & 0 & 5 & 5365.1825 & 0.0020 \\
5 & 2 & 3 & $\leftarrow$ & 4 & 1 & 3 & 5047.9828 & 0.0070 \\
& & & & & & & & \\
& & & & & & & RMS & $4.868 \mathrm{kHz}$ \\
\hline
\end{tabular}


Table S31: Measured rotational transitions $\left(\nu_{\mathrm{obs}}\right)$ for the $3 \mathrm{w}-{ }^{18} \mathrm{O} 1{ }^{18} \mathrm{O} 2{ }^{18} \mathrm{O} 3$ complex of camphor with three water molecules, and the residuals $\left(\nu_{\text {obs }}-\nu_{\text {calc }}\right)$ for the fit reported in Table S22 (see Figure S5 for oxygen atom numbering).

\begin{tabular}{lllllllll}
\hline$J^{\prime}$ & $K_{a}^{\prime}$ & $K_{c}^{\prime}$ & $\leftarrow$ & $J^{\prime \prime}$ & $K_{a}^{\prime \prime}$ & $K_{c}^{\prime \prime}$ & $\nu_{\mathrm{obs}}(\mathrm{MHz})$ & $\nu_{\mathrm{obs}}-\nu_{\text {calc }}$ \\
\hline
\end{tabular}

$a$-type transitions:

$\begin{array}{ccccccccc}3 & 1 & 3 & \leftarrow & 2 & 1 & 2 & 2083.8669 & -0.0016 \\ 3 & 1 & 2 & \leftarrow & 2 & 1 & 1 & 2216.6128 & -0.0005 \\ & & & & & & & & \\ 4 & 1 & 4 & \leftarrow & 3 & 1 & 3 & 2775.3431 & -0.0064 \\ 4 & 0 & 4 & \leftarrow & 3 & 0 & 3 & 2840.9893 & -0.0026 \\ 4 & 2 & 3 & \leftarrow & 3 & 2 & 2 & 2867.1578 & 0.0109 \\ 4 & 2 & 2 & \leftarrow & 3 & 2 & 1 & 2895.5583 & -0.0030 \\ 4 & 1 & 3 & \leftarrow & 3 & 1 & 2 & 2951.7833 & -0.0001 \\ & & & & & & & & \\ 5 & 1 & 5 & \leftarrow & 4 & 1 & 4 & 3464.4751 & 0.0007 \\ 5 & 0 & 5 & \leftarrow & 4 & 0 & 4 & 3531.9074 & -0.0039 \\ 5 & 2 & 4 & \leftarrow & 4 & 2 & 3 & 3580.2761 & -0.0030 \\ 5 & 3 & 3 & \leftarrow & 4 & 3 & 2 & 3595.5795 & 0.0003 \\ 5 & 3 & 2 & \leftarrow & 4 & 3 & 1 & 3598.0793 & -0.0025 \\ & & & & & & & & \\ 6 & 1 & 6 & \leftarrow & 5 & 1 & 5 & 4151.0350 & -0.0037 \\ 6 & 0 & 6 & \leftarrow & 5 & 0 & 5 & 4213.5570 & -0.0035 \\ 6 & 2 & 5 & \leftarrow & 5 & 2 & 4 & 4291.0025 & -0.0016 \\ 6 & 3 & 4 & \leftarrow & 5 & 3 & 3 & 4316.9618 & -0.0004 \\ 6 & 3 & 3 & \leftarrow & 5 & 3 & 2 & 4323.5616 & -0.0011 \\ 6 & 2 & 4 & \leftarrow & 5 & 2 & 3 & 4381.3693 & -0.0001 \\ 6 & 1 & 5 & \leftarrow & 5 & 1 & 4 & 4410.1667 & -0.0001 \\ & & & & & & & & \\ 7 & 0 & 7 & \leftarrow & 6 & 0 & 6 & 4888.0125 & -0.0008 \\ 7 & 2 & 6 & \leftarrow & 6 & 2 & 5 & 4998.8933 & 0.0005 \\ 7 & 4 & 4 & \leftarrow & 6 & 4 & 3 & 5035.7554 & 0.0043 \\ 7 & 4 & 3 & \leftarrow & 6 & 4 & 2 & 5036.2382 & -0.0010 \\ 7 & 3 & 5 & \leftarrow & 6 & 3 & 4 & 5038.6980 & -0.0018 \\ 7 & 3 & 4 & \leftarrow & 6 & 3 & 3 & 5053.2771 & -0.0003 \\ 7 & 1 & 6 & \leftarrow & 6 & 1 & 5 & 5130.4625 & -0.0033 \\ 7 & 2 & 5 & \leftarrow & 6 & 2 & 4 & 5131.7765 & -0.0006 \\ & & & & & & & & \\ 8 & 1 & 8 & \leftarrow & 7 & 1 & 7 & 5516.6166 & 0.0018 \\ 8 & 0 & 8 & \leftarrow & 7 & 0 & 7 & 5558.2169 & -0.0010 \\ 8 & 2 & 7 & \leftarrow & 7 & 2 & 6 & 5703.5714 & 0.0005 \\ 8 & 4 & 5 & \leftarrow & 7 & 4 & 4 & 5758.6696 & 0.0047 \\ 8 & 4 & 4 & \leftarrow & 7 & 4 & 3 & 5759.9900 & -0.0061 \\ 8 & 3 & 6 & \leftarrow & 7 & 3 & 5 & 5760.2617 & -0.0021 \\ 8 & 3 & 5 & \leftarrow & 7 & 3 & 4 & 5788.5697 & -0.0018 \\ 8 & 1 & 7 & \leftarrow & 7 & 1 & 6 & 5842.5837 & -0.0020 \\ 8 & 2 & 6 & \leftarrow & 7 & 2 & 5 & 5882.7023 & -0.0028\end{array}$




$\begin{array}{ccccccccc}9 & 1 & 9 & \leftarrow & 8 & 1 & 8 & 6196.0736 & 0.0034 \\ 9 & 0 & 9 & \leftarrow & 8 & 0 & 8 & 6226.7964 & 0.0010 \\ 9 & 2 & 8 & \leftarrow & 8 & 2 & 7 & 6404.7488 & 0.0027 \\ 9 & 5 & 4 & \leftarrow & 5 & 5 & 3 & 6475.2991 & 0.0062 \\ 9 & 3 & 7 & \leftarrow & 8 & 3 & 6 & 6481.0065 & 0.0007 \\ 9 & 4 & 6 & \leftarrow & 8 & 4 & 5 & 6482.7119 & 0.0043 \\ 9 & 4 & 5 & \leftarrow & 8 & 4 & 4 & 6485.8587 & -0.0047 \\ 9 & 3 & 6 & \leftarrow & 8 & 3 & 5 & 6530.6384 & -0.0025 \\ 9 & 1 & 8 & \leftarrow & 8 & 1 & 7 & 6544.9369 & -0.0030 \\ 9 & 2 & 7 & \leftarrow & 8 & 2 & 6 & 6630.7553 & -0.0027 \\ & & & & & & & & \\ 10 & 1 & 10 & \leftarrow & 5 & 1 & 9 & 6873.7503 & 0.0001 \\ 10 & 0 & 10 & \leftarrow & 5 & 0 & 9 & 6895.4059 & 0.0150 \\ 10 & 2 & 9 & \leftarrow & 5 & 2 & 8 & 7102.2341 & -0.0010 \\ 10 & 4 & 7 & \leftarrow & 9 & 4 & 6 & 7207.7585 & -0.0047 \\ 10 & 1 & 9 & \leftarrow & 5 & 1 & 8 & 7236.5891 & 0.0012 \\ 10 & 3 & 7 & \leftarrow & 9 & 3 & 6 & 7280.0101 & -0.0040 \\ 10 & 2 & 8 & \leftarrow & 5 & 2 & 7 & 7373.3380 & -0.0088\end{array}$

$c$-type transitions:

\begin{tabular}{ccccccccc}
5 & 3 & 2 & $\leftarrow$ & 4 & 2 & 3 & 6128.1287 & 0.0013 \\
4 & 3 & 1 & $\leftarrow$ & 3 & 2 & 2 & 5397.1815 & -0.0107 \\
4 & 2 & 2 & $\leftarrow$ & 3 & 1 & 2 & 4290.8892 & 0.0085 \\
5 & 1 & 4 & $\leftarrow$ & 4 & 0 & 4 & 4443.6278 & 0.0166 \\
4 & 2 & 3 & $\leftarrow$ & 3 & 1 & 3 & 4513.5911 & 0.0073 \\
3 & 3 & 0 & $\leftarrow$ & 2 & 2 & 0 & 4670.7199 & 0.0016 \\
3 & 3 & 1 & $\leftarrow$ & 2 & 2 & 1 & 4673.5096 & -0.0034 \\
4 & 3 & 1 & $\leftarrow$ & 3 & 2 & 1 & 5382.6994 & -0.0010 \\
4 & 3 & 2 & $\leftarrow$ & 3 & 2 & 2 & 5396.3492 & -0.0035 \\
6 & 2 & 4 & $\leftarrow$ & 5 & 1 & 4 & 5672.1410 & 0.0053 \\
5 & 3 & 2 & $\leftarrow$ & 4 & 2 & 2 & 6085.2217 & 0.0009 \\
5 & 3 & 3 & $\leftarrow$ & 4 & 2 & 3 & 6124.7798 & -0.0054 \\
6 & 2 & 5 & $\leftarrow$ & 5 & 1 & 5 & 6145.0551 & 0.0117 \\
7 & 1 & 6 & $\leftarrow$ & 6 & 0 & 6 & 6238.7691 & -0.0029 \\
7 & 2 & 5 & $\leftarrow$ & 6 & 1 & 5 & 6393.7535 & 0.0076 \\
6 & 3 & 3 & $\leftarrow$ & 5 & 2 & 3 & 6773.6975 & -0.0039 \\
6 & 3 & 4 & $\leftarrow$ & 5 & 2 & 4 & 6861.4650 & -0.0033 \\
8 & 2 & 6 & $\leftarrow$ & 7 & 1 & 6 & 7145.9900 & 0.0047 \\
7 & 3 & 4 & $\leftarrow$ & 6 & 2 & 4 & 7445.6082 & -0.0013 \\
& & & & & & & & \\
& & & & & & & $\mathrm{RMS}$ & $4.856 \mathrm{kHz}$ \\
\hline
\end{tabular}


Table S32: The abbreviated results of the fit of the $r_{0}$ geometry of $1 \mathrm{w}(\mathrm{I})$ complex of camphor with one water molecule to 6 rotational constants from 2 different isotopic species.

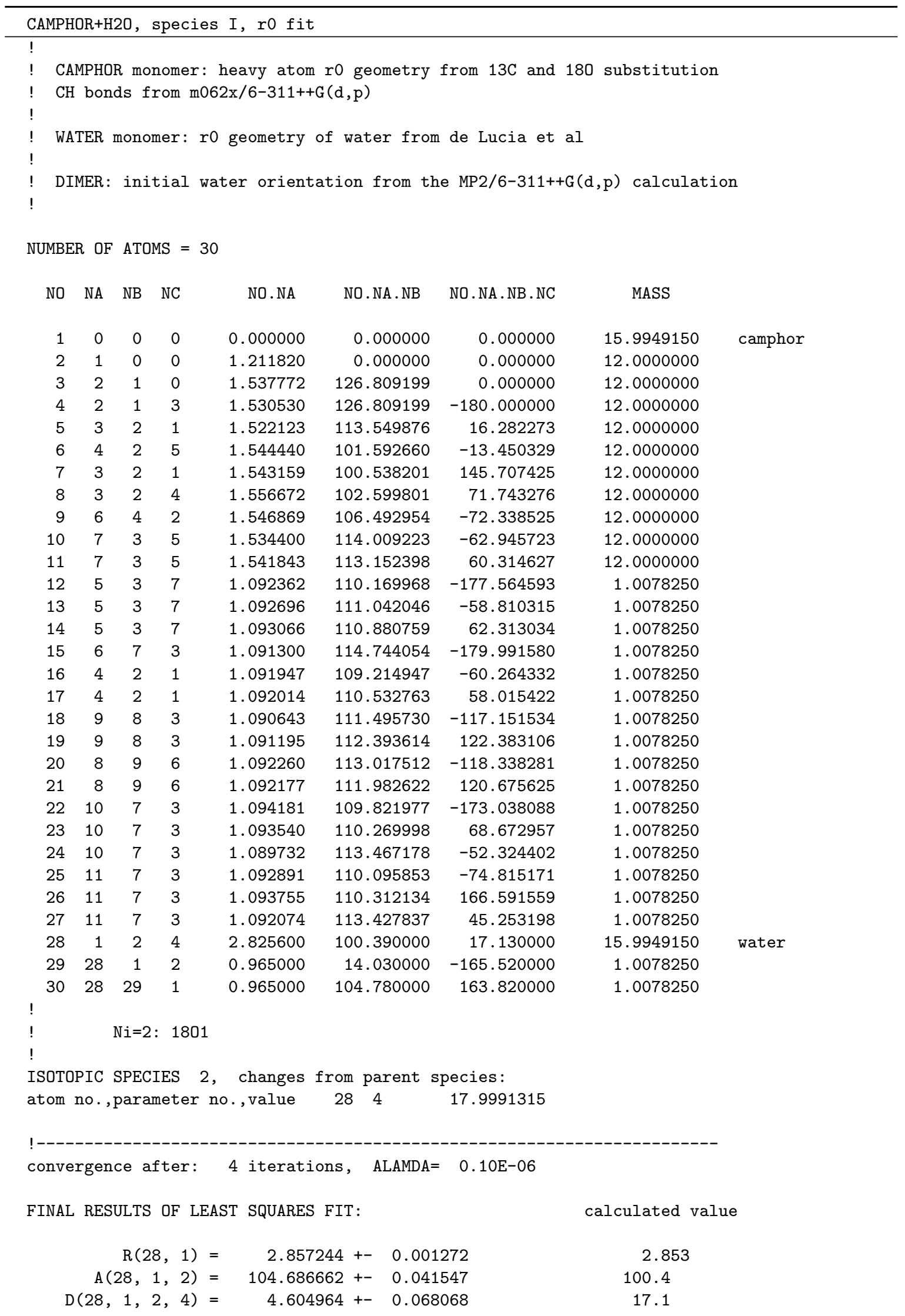




\begin{tabular}{|c|c|c|c|c|c|c|c|}
\hline & & Chi- & quared = & 0.010 & & & \\
\hline & & Deviatior & of fit $=$ & 0.059 & & & \\
\hline $\mathrm{Ni}$ & Axis & Iobs & Icalc & Io-c & Bobs & Bcalc & $\mathrm{Bo}-\mathrm{C}$ \\
\hline 1 & a & 375.84316 & 375.77688 & 0.06628 & 1344.6540 & 1344.8912 & -0.2372 \\
\hline 1 & $\mathrm{~b}$ & 762.27461 & 762.25083 & 0.02378 & 662.9881 & 663.0088 & -0.0207 \\
\hline 1 & c & 792.24728 & 792.22106 & 0.02622 & 637.9056 & 637.9268 & -0.0211 \\
\hline 2 & a & 376.27998 & 376.34355 & -0.06357 & 1343.0930 & 1342.8661 & 0.2269 \\
\hline 2 & $\mathrm{~b}$ & 795.67730 & 795.69863 & -0.02133 & 635.1558 & 635.1387 & 0.0170 \\
\hline 2 & c & 825.78699 & 825.81118 & -0.02419 & 611.9968 & 611.9789 & 0.0179 \\
\hline
\end{tabular}

Principal coordinates and estimated uncertainties:

$\begin{array}{llllllll}\text { ATOM NO. } & \mathrm{A} & \mathrm{dA} & \mathrm{B} & \mathrm{dB} & \mathrm{C} & \mathrm{d}\end{array}$
$\begin{array}{ll}-1.81106 & 0.00053\end{array}$
$1.14268 \quad 0.00131$
$0.33101 \quad 0.00089$
$\begin{array}{ll}-0.86921 & 0.00018\end{array}$
$0.38952 \quad 0.00080$
$0.21200 \quad 0.00062$
$0.61962 \quad 0.00035$
$0.74594 \quad 0.00025$
$0.35728 \quad 0.00055$
$\begin{array}{ll}-0.92557 & 0.00055\end{array}$
$\begin{array}{ll}-1.10511 & 0.00072\end{array}$
$2.24129 \quad 0.00022$
$-0.11271 \quad 0.00152$
$0.89619 \quad 0.00108$
$0.29167 \quad 0.00176$
$0.57295 \quad 0.00074$
$\begin{array}{ll}-1.47894 & 0.00009\end{array}$
$\begin{array}{ll}1.23988 & 0.00037\end{array}$
$\begin{array}{ll}-0.19913 & 0.00072\end{array}$
$\begin{array}{ll}-0.11491 & 0.00109\end{array}$
$\begin{array}{ll}1.00078 & 0.00091\end{array}$
$\begin{array}{ll}0.06901 & 0.00117\end{array}$
$\begin{array}{ll}-0.69317 & 0.00026\end{array}$
$1.03344 \quad 0.00121$
$\begin{array}{lll}-1.45605 & 0.00093\end{array}$
$2.77365 \quad 0.00036$
$\begin{array}{ll}-0.23686 & 0.00139\end{array}$
$0.80528 \quad 0.00119$
$0.12907 \quad 0.00161$
$0.34716 \quad 0.00131$
$2.76083 \quad 0.00083$
0.567840 .00150
$\begin{array}{ll}2.65950 & 0.00048\end{array}$
$\begin{array}{ll}1.96234 & 0.00117\end{array}$
$2.44624 \quad 0.00066$
$0.80998 \quad 0.00116$
$\begin{array}{lll}-2.39953 & 0.00054\end{array}$
$\begin{array}{ll}-1.40916 & 0.00072\end{array}$
$\begin{array}{ll}-1.24435 & 0.00100\end{array}$
$\begin{array}{ll}-1.51939 & 0.00100\end{array}$
$-1.63706 \quad 0.00130$
$\begin{array}{ll}1.70624 & 0.00026\end{array}$
$1.36165 \quad 0.00090$
$-0.670730 .00106$
$\begin{array}{ll}-2.13563 & 0.00019\end{array}$
$\begin{array}{ll}1.08028 & 0.00191\end{array}$
$\begin{array}{ll}-0.66294 & 0.00193\end{array}$
$0.41858 \quad 0.00238$
$\begin{array}{ll}-0.65089 & 0.00167\end{array}$
$\begin{array}{ll}-1.08178 & 0.00186\end{array}$
$2.03095 \quad 0.00144$
$-1.87537$
$0.63355 \quad 0.00218$
$\begin{array}{ll}0.35017 & 0.00172\end{array}$
$\begin{array}{ll}-2.03734 & 0.00149\end{array}$
$\begin{array}{ll}1.49825 & 0.00095\end{array}$
$0.29856 \quad 0.00131$
$0.31096 \quad 0.00188$
$\begin{array}{ll}1.98289 & 0.00159\end{array}$
$\begin{array}{ll}1.98030 & 0.00103\end{array}$
$0.43903 \quad 0.00136$
$3.13568 \quad 0.00076$
$\begin{array}{ll}-1.01356 & 0.00192\end{array}$
$3.18117 \quad 0.00076$
0.717790 .00176
$3.18837 \quad 0.00033$
$\begin{array}{ll}-0.43904 & 0.00126\end{array}$
$1.32284 \quad 0.00161$
$\begin{array}{ll}1.02433 & 0.00200\end{array}$
$\begin{array}{ll}1.06900 & 0.00146\end{array}$
$\begin{array}{ll}-0.69245 & 0.00216\end{array}$
$0.31599 \quad 0.00151$
$\begin{array}{ll}-0.26613 & 0.00128\end{array}$
$\begin{array}{ll}-4.09320 & 0.00019\end{array}$
$\begin{array}{lll}-3.48557 & 0.00029\end{array}$
$\begin{array}{ll}-4.95720 & 0.00023\end{array}$
$-0.43929 \quad 0.00045$
$0.24166 \quad 0.00021$
$\begin{array}{ll}-0.00979 & 0.00067\end{array}$
2.507110 .00015
$2.01684 \quad 0.00101$
$\begin{array}{ll}-1.35110 & 0.00097\end{array}$
$\begin{array}{ll}-1.01480 & 0.00182\end{array}$
0.316510 .00119
$\begin{array}{ll}-2.48925 & 0.00107\end{array}$
$\begin{array}{ll}-2.80784 & 0.00036\end{array}$
$\begin{array}{ll}-2.23441 & 0.00034\end{array}$
$\begin{array}{ll}-0.34210 & 0.00039\end{array}$
$\begin{array}{ll}-0.02852 & 0.00026\end{array}$
$\begin{array}{ll}-0.35814 & 0.00033\end{array}$
। ।

NOTES: 1/ only the uncertainties for those coordinates which are completely defined by the fitted internals should be trusted

2/ the uncertainties are somewhat limited by the linear approximation coord $=(d$ coord/d parameter $) *$ parameter used for evaluation

$3 /$ only the effect of the internals $R, A$, and D is propagated 
Table S33: The abbreviated results of the fit of the $r_{0}$ geometry of $1 \mathrm{w}$ (II) complex of camphor with one water molecule to 6 rotational constants from 2 different isotopic species.

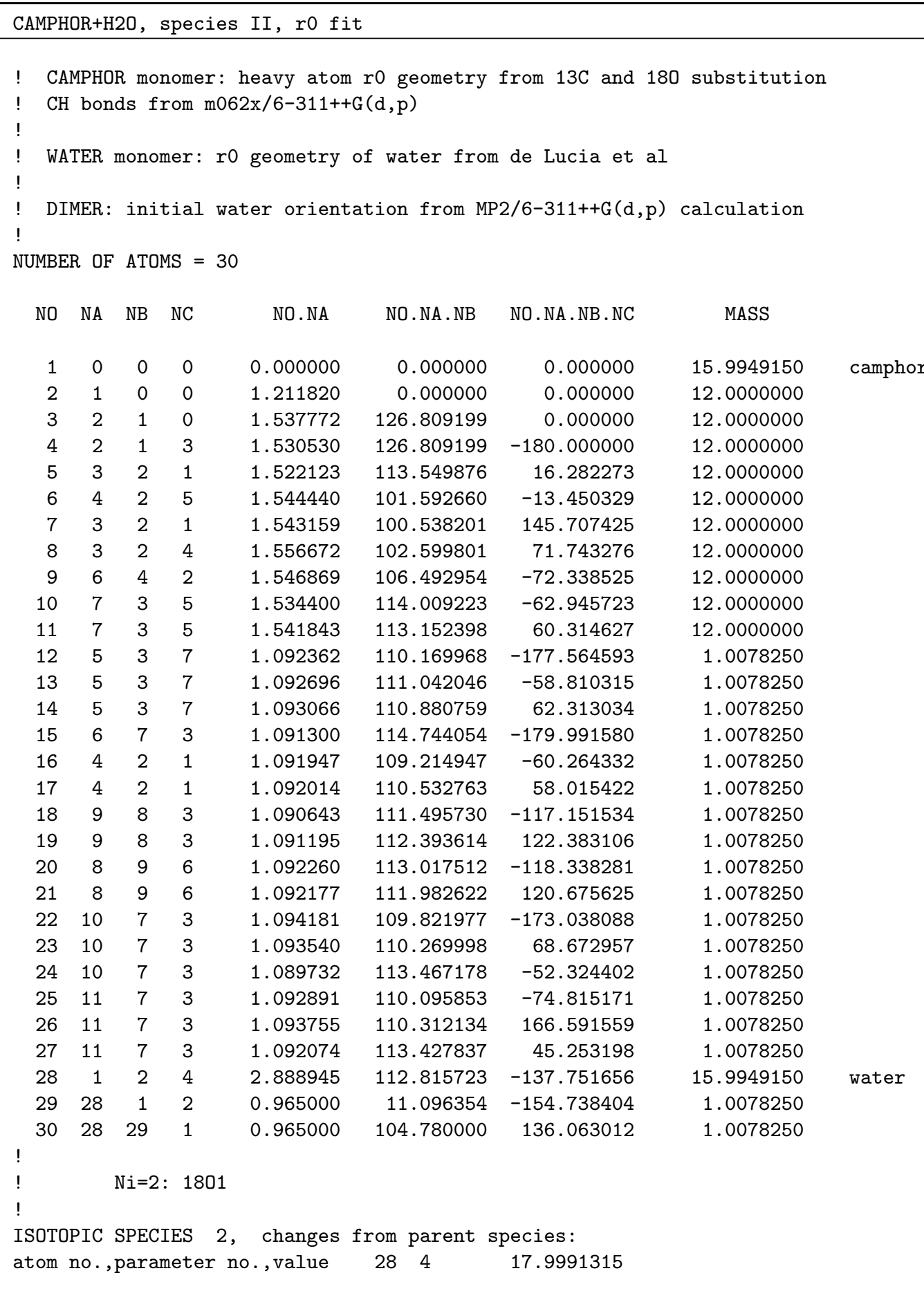

converged after: 5 iterations, ALAMDA $=0.10 \mathrm{E}-07$

FINAL RESULTS OF LEAST SQUARES FIT:

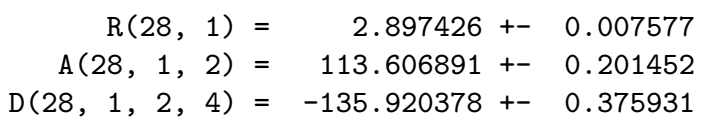

calculated value 2.889

112.8

$-137.8$ 


\begin{tabular}{|c|c|c|c|c|c|c|c|}
\hline & & Chi- & quared $=$ & 0.367 & & & \\
\hline & & Deviation & of fit $=$ & 0.350 & & & \\
\hline $\mathrm{Ni}$ & Axis & Iobs & Icalc & Io-c & Bobs & Bcalc & Bo-c \\
\hline 1 & a & 377.19637 & 377.27851 & -0.08214 & 1339.8300 & 1339.5383 & 0.2917 \\
\hline 1 & $\mathrm{~b}$ & 733.45950 & 733.20686 & 0.25264 & 689.0347 & 689.2721 & -0.2374 \\
\hline 1 & c & 750.98137 & 750.62406 & 0.35730 & 672.9581 & 673.2785 & -0.3203 \\
\hline 2 & $\mathrm{a}$ & 378.07112 & 377.96753 & 0.10359 & 1336.7300 & 1337.0964 & -0.3664 \\
\hline 2 & $\mathrm{~b}$ & 763.09497 & 763.32177 & -0.22680 & 662.2754 & 662.0786 & 0.1968 \\
\hline 2 & c & 780.33533 & 780.66262 & -0.32729 & 647.6434 & 647.3719 & 0.2715 \\
\hline
\end{tabular}

Correlation coefficients:

$\begin{array}{rrrrr} & & 1 & 2 & 3 \\ 1: & \mathrm{R}(28,1) & 1.000 & & \\ 2: & \mathrm{A}(28,1,2) & -0.910 & 1.000 & \\ 3: & \mathrm{D}(28,1,2,4) & -0.133 & -0.242 & 1.000\end{array}$

Principal coordinates and estimated uncertainties:

\begin{tabular}{|c|c|c|c|c|c|c|}
\hline ATOM NO. & A & $\mathrm{dA}$ & B & $\mathrm{dB}$ & $\mathrm{C}$ & $\mathrm{dC}$ \\
\hline 1 & -1.74968 & 0.00308 & -0.78740 & 0.01398 & -1.11267 & 0.00627 \\
\hline 2 & -0.65709 & 0.00216 & -0.70662 & 0.00740 & -0.59475 & 0.00468 \\
\hline 3 & -0.05388 & 0.00165 & 0.52778 & 0.00143 & 0.09600 & 0.00476 \\
\hline 4 & 0.39612 & 0.00529 & -1.81296 & 0.00392 & -0.49840 & 0.01284 \\
\hline 5 & -0.77744 & 0.00596 & 1.82501 & 0.00543 & -0.23644 & 0.01504 \\
\hline 6 & 1.53070 & 0.00366 & -1.09569 & 0.00464 & 0.26552 & 0.00928 \\
\hline 7 & 1.43468 & 0.00154 & 0.35914 & 0.00197 & -0.27424 & 0.00261 \\
\hline 8 & -0.08869 & 0.00420 & 0.12641 & 0.01021 & 1.59963 & 0.00229 \\
\hline 9 & 1.04699 & 0.00620 & -0.94116 & 0.01358 & 1.72666 & 0.00741 \\
\hline 10 & 2.37049 & 0.00404 & 1.34567 & 0.00823 & 0.43668 & 0.00873 \\
\hline 11 & 1.69994 & 0.00538 & 0.47768 & 0.01062 & -1.78847 & 0.00345 \\
\hline 12 & -1.82788 & 0.00547 & 1.75578 & 0.00708 & 0.05518 & 0.01644 \\
\hline 13 & -0.75055 & 0.00814 & 2.02517 & 0.01259 & -1.31031 & 0.01641 \\
\hline 14 & -0.32600 & 0.00814 & 2.66916 & 0.00190 & 0.29120 & 0.02035 \\
\hline 15 & 2.51305 & 0.00495 & -1.55876 & 0.00699 & 0.15834 & 0.01425 \\
\hline 16 & 0.68103 & 0.00643 & -2.12654 & 0.01052 & -1.50480 & 0.01543 \\
\hline 17 & -0.01060 & 0.00831 & -2.68507 & 0.00162 & 0.01783 & 0.01869 \\
\hline 18 & 1.83765 & 0.00724 & -0.60551 & 0.02032 & 2.39876 & 0.00657 \\
\hline 19 & 0.68009 & 0.00935 & -1.89488 & 0.01549 & 2.10942 & 0.01376 \\
\hline 20 & -1.06620 & 0.00541 & -0.25118 & 0.01077 & 1.90776 & 0.00389 \\
\hline 21 & 0.11571 & 0.00576 & 1.01439 & 0.01493 & 2.20176 & 0.00790 \\
\hline 22 & 3.41233 & 0.00331 & 1.08835 & 0.01014 & 0.22317 & 0.00817 \\
\hline 23 & 2.20053 & 0.00734 & 2.36132 & 0.00553 & 0.06870 & 0.01560 \\
\hline 24 & 2.24572 & 0.00490 & 1.35891 & 0.01516 & 1.51916 & 0.00880 \\
\hline 25 & 1.63777 & 0.00840 & 1.52343 & 0.01292 & -2.09985 & 0.01006 \\
\hline 26 & 2.70611 & 0.00547 & 0.12108 & 0.01090 & -2.02673 & 0.00536 \\
\hline 27 & 0.98913 & 0.00623 & -0.08321 & 0.01630 & -2.39902 & 0.00148 \\
\hline 28 & -3.87057 & 0.00118 & 0.40482 & 0.00521 & 0.46070 & 0.00301 \\
\hline 29 & -3.27686 & 0.00150 & 0.04216 & 0.00223 & -0.20803 & 0.00087 \\
\hline 30 & -4.74248 & 0.00125 & 0.06382 & $\begin{array}{c}0.00297 \\
\mid\end{array}$ & 0.22678 & $\begin{array}{c}0.00220 \\
\mid\end{array}$ \\
\hline
\end{tabular}

NOTES: 1/ only the uncertainties for those coordinates which are completely defined by the fitted internals should be trusted

2/ the uncertainties are somewhat limited by the linear approximation coord $=(\mathrm{d}$ coord/d parameter $) *$ parameter used for evaluation

3 / only the effect of the internals R, A, and D is propagated 
Table S34: The abbreviated results of the fit of the $r_{0}$ geometry of $2 \mathrm{w}(\mathrm{I})$ complex of camphor with two water molecules to 12 rotational constants from 4 different isotopic species.

CAMPHOR+(H2O) 2 , species I, rO fit

! CAMPHOR monomer: heavy atom r0 geometry from $13 \mathrm{C}$ and 180 substitution

$\mathrm{CH}$ bonds from $\mathrm{m} 062 \mathrm{x} / 6-311++\mathrm{G}(\mathrm{d}, \mathrm{p})$

WATER monomer: $r 0$ geometry of water from de Lucia et al

CLUSTER: initial water orientations from mp2/6-311++g $(d, p)$
NO.NA

0.000000

1. 211820

1. 537772

1. 530530

1.522123

1.544440

1.543159

1. 556672

1.546869

1. 534400

1. 541843

1. 092362

1.092696

1.093066

1. 091300

1. 091947

1. 092014

1. 090643

1. 091195

1. 092260

1. 092177

1. 094181

1. 093540

1. 089732

1. 092891

1. 093755

1. 092074

2. 793777

0.965000

0.965000

2.801300

0.965000

0.965000
NO.NA.NB

0.000000

0.000000

126.809199

126.809199

113.549876

101.592660

100.538201

102.599801

106.492954

114.009223

113.152398

110.169968

111.042046

110.880759

114.744054

109. 214947

110.532763

111.495730

112.393614

113.017512

111.982622

109.821977

110.269998

113.467178

110.095853

110.312134

113.427837

118.616844

8.875916

104.780000

84.844072

12.121476

104.780000
NO.NA . NB . NC

0.000000

0.000000

0.000000

$-180.000000$

16.282273

$-13.450329$

145.707425

71.743276

$-72.338525$

$-62.945723$

60.314627

$-177.564593$

$-58.810315$

62.313034

$-179.991580$

$-60.264332$

58.015422

$-117.151534$

122.383106

$-118.338281$

120.675625

$-173.038088$

68.672957

$-52.324402$

$-74.815171$

166.591559

45.253198

24.649903

$-128.688897$

118.333579

35.405494

161.745442

$-163.932918$
MASS

15.9949150

12.0000000

12.0000000

12.0000000

12.0000000

12.0000000

12.0000000

12.0000000

12.0000000

12.0000000

12.0000000

1.0078250

1.0078250

1.0078250

1. 0078250

1.0078250

1.0078250

1.0078250

1.0078250

1.0078250

1. 0078250

1.0078250

1.0078250

1. 0078250

1.0078250

1.0078250

1.0078250

15.9949150

1.0078250

1.0078250

15.9949150

1.0078250

1.0078250 !

! $\mathrm{Ni}=2: 1802$ /smaller $\mathrm{Pb} /$

ISOTOPIC SPECIES 2, changes from parent species:

atom no.,parameter no., value $\quad \begin{array}{lll}28 & 4 & 17.9991315\end{array}$

!

! $\mathrm{Ni}=3: 1801$ /larger $\mathrm{Pb}, \mathrm{H}$-bonded to $\mathrm{C}=0$ /

ISOTOPIC SPECIES 3, changes from parent species:

atom no., parameter no., value $\quad \begin{array}{lll}31 & 4 & 17.9991315\end{array}$ !

! Ni=4: 18011802 
ISOTOPIC SPECIES 4, changes from parent species:

atom no., parameter no., value $\quad \begin{array}{lll}28 & 4 & 17.9991315\end{array}$

atom no.,parameter no., value $\quad 31 \quad 4 \quad 17.9991315$

convergence after: 9 iterations, $\mathrm{ALAMDA}=0.10 \mathrm{E}-11$

FINAL RESULTS OF LEAST SQUARES FIT:

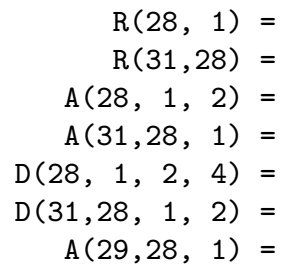

calculated value
2.794
2.801
118.6
84.8
24.6
35.4
8.9 \\ 0.006369 \\ Chi-squared = \\ Deviation of fit $=$
}

Correlation coefficients:

$\begin{array}{lrrrrrrrr} & & 1 & 2 & 3 & 4 & 5 & 6 & 7 \\ 1: & \mathrm{R}(28,1) & 1.000 & & & & & & \\ 2: & \mathrm{R}(31,28) & -0.686 & 1.000 & & & & & \\ 3: & \mathrm{A}(28,1,2) & -0.924 & 0.425 & 1.000 & & & & \\ 4: & \mathrm{A}(31,28,1) & 0.905 & -0.425 & -0.995 & 1.000 & & & \\ 5: & \mathrm{D}(28,1,2,4) & -0.719 & 0.052 & 0.924 & -0.923 & 1.000 & & \\ 6: & \mathrm{D}(31,28,1,2) & -0.823 & 0.225 & 0.974 & -0.974 & 0.983 & 1.000 & \\ 7: & \mathrm{A}(29,28,1) & -0.135 & -0.289 & 0.124 & -0.105 & 0.209 & 0.162 & 1.000\end{array}$

Principal coordinates and estimated uncertainties:

$\begin{array}{rrcrcrc}\text { ATOM NO. } & \mathrm{A} & \mathrm{dA} & \mathrm{B} & \mathrm{dB} & \mathrm{C} & \mathrm{dC} \\ & & & & & & \\ 1 & -1.25436 & 0.00241 & 1.49367 & 0.00581 & 0.51885 & 0.00688 \\ 2 & -0.38441 & 0.00085 & 0.80418 & 0.00129 & 0.03274 & 0.00262 \\ 3 & 1.04810 & 0.00369 & 0.62004 & 0.00924 & 0.56072 & 0.01093 \\ 4 & -0.49353 & 0.00956 & -0.05604 & 0.02153 & -1.22846 & 0.01210 \\ 5 & 1.22845 & 0.01446 & 1.07598 & 0.03267 & 2.00171 & 0.01969 \\ 6 & 0.92442 & 0.00948 & -0.66213 & 0.02112 & -1.31438 & 0.01070 \\ 7 & 1.30126 & 0.00259 & -0.85426 & 0.00346 & 0.18167 & 0.01083 \\ 8 & 1.89796 & 0.00558 & 1.40132 & 0.00655 & -0.48358 & 0.03020 \\ 9 & 1.86654 & 0.01435 & 0.48471 & 0.02697 & -1.75026 & 0.01519 \\ 10 & 2.74816 & 0.00491 & -1.31562 & 0.00886 & 0.40075 & 0.00688\end{array}$




$\begin{array}{rrrrrrr}11 & 0.38204 & 0.00974 & -1.83937 & 0.01437 & 0.93126 & 0.03393 \\ 12 & 0.96738 & 0.01393 & 2.13231 & 0.03389 & 2.09801 & 0.03465 \\ 13 & 0.57438 & 0.02045 & 0.51662 & 0.04269 & 2.67499 & 0.00553 \\ 14 & 2.26408 & 0.01717 & 0.94384 & 0.03919 & 2.32543 & 0.02571 \\ 15 & 0.99559 & 0.01316 & -1.56517 & 0.03084 & -1.92297 & 0.02468 \\ 16 & -1.27719 & 0.00752 & -0.80321 & 0.02026 & -1.08715 & 0.03030 \\ 17 & -0.77175 & 0.01709 & 0.55766 & 0.03572 & -2.08779 & 0.00440 \\ 18 & 2.86463 & 0.01597 & 0.13270 & 0.02998 & -2.01370 & 0.01737 \\ 19 & 1.46303 & 0.02188 & 0.99773 & 0.04155 & -2.62473 & 0.02027 \\ 20 & 1.50796 & 0.00838 & 2.40399 & 0.01005 & -0.67219 & 0.04328 \\ 21 & 2.90979 & 0.00272 & 1.52028 & 0.00163 & -0.09002 & 0.04008 \\ 22 & 2.88110 & 0.00325 & -2.32413 & 0.00274 & -0.00232 & 0.02207 \\ 23 & 2.97515 & 0.01333 & -1.35611 & 0.02632 & 1.46971 & 0.00574 \\ 24 & 3.48638 & 0.00051 & -0.66805 & 0.00262 & -0.07169 & 0.00937 \\ 25 & 0.71110 & 0.01801 & -1.94523 & 0.03148 & 1.96804 & 0.03304 \\ 26 & 0.42732 & 0.00746 & -2.82885 & 0.00696 & 0.46739 & 0.04951 \\ 27 & -0.66351 & 0.00952 & -1.52492 & 0.01348 & 0.95546 & 0.03709 \\ 28 & -3.87159 & 0.00035 & 0.88162 & 0.00133 & -0.19617 & 0.00125 \\ 29 & -3.16068 & 0.00603 & 1.52692 & 0.00708 & -0.09911 & 0.00611 \\ 30 & -4.56758 & 0.00190 & 1.19111 & 0.00775 & 0.39632 & 0.00136 \\ 31 & -3.15315 & 0.00043 & -1.83278 & 0.00066 & 0.07777 & 0.00745 \\ 32 & -3.58924 & 0.00036 & -0.97557 & 0.00071 & -0.00128 & 0.00599 \\ 33 & -3.85026 & 0.00061 & -2.47965 & 0.00084 & -0.08597 & 0.01105 \\ & & 1 & & 1 & & 1\end{array}$

NOTES: 1/ only the uncertainties for those coordinates which are completely defined by the fitted internals should be trusted

2/ the uncertainties are somewhat limited by the linear approximation $\operatorname{coord}=(d$ coord $/ d$ parameter $) *$ parameter used for evaluation

$3 /$ only the effect of the internals $R, A$, and D is propagated 
Table S35: The abbreviated results of fit of the $r_{0}$ geometry of $2 \mathrm{w}$ (II) complex of camphor with two water molecules to 12 rotational constants from 4 different isotopic species.

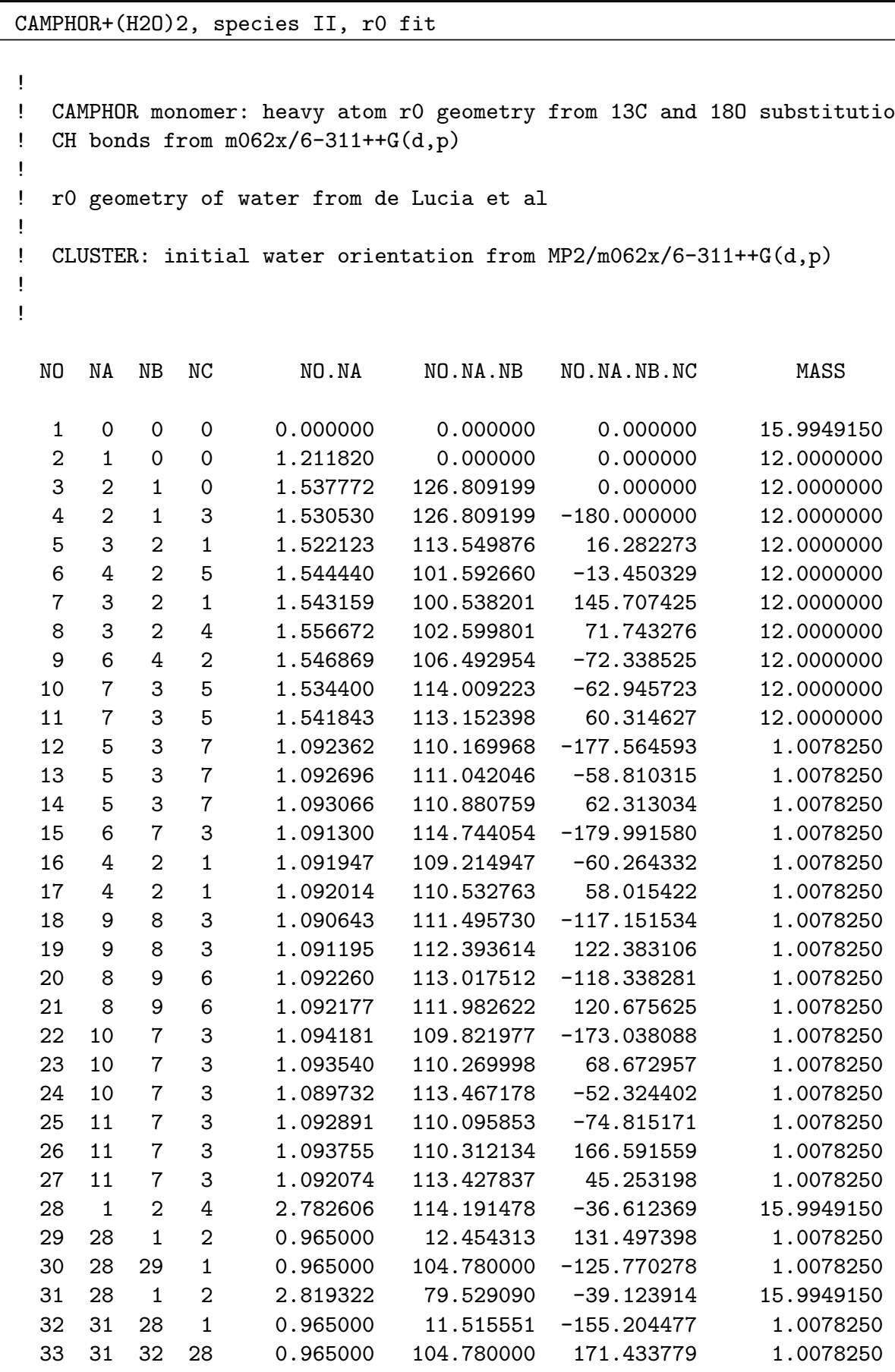

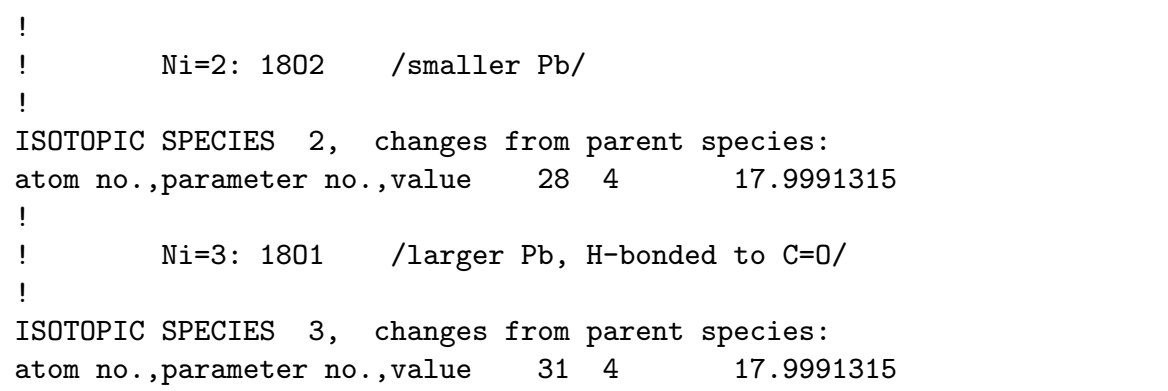


ISOTOPIC SPECIES 4, changes from parent species:

$\begin{array}{llll}\text { atom no., parameter no., value } & 28 & 4 & 17.9991315 \\ \text { atom no., parameter no., value } & 31 & 4 & 17.9991315\end{array}$

convergence after: 13 iterations, $\quad$ ALAMDA $=0.10 \mathrm{E}-07$

FINAL RESULTS OF LEAST SQUARES FIT:

$$
\begin{array}{r}
\mathrm{R}(28,1)= \\
\mathrm{R}(31,28)= \\
\mathrm{A}(28,1,2)= \\
\mathrm{A}(31,28,1)= \\
\mathrm{D}(28,1,2,4)= \\
\mathrm{D}(31,28,1,2)= \\
\mathrm{A}(29,28,1)=
\end{array}
$$$$
\begin{array}{r}
2 . \\
2.87 \\
112 . \\
82 . \\
-31 . \\
-41 . \\
33 . \\
\text { it }=
\end{array}
$$$$
2.777603+-0.001080
$$$$
2.837303+-0.000679
$$$$
112.415378+-0.043599
$$$$
82.172912+-0.036761
$$$$
-31.953513+-0.159855
$$$$
-41.468353+-0.041081
$$$$
33.810927+-0.279780
$$

calculated value

$$
2.783
$$$$
2.819
$$

114.19

79.53

$-36.6$

$-39.1$

12.45

$\begin{aligned} \text { Chi-squared }= & 0.0000518045 \\ \text { Deviation of fit }= & 0.003219\end{aligned}$

\begin{tabular}{llccr} 
Ni & Axis & Iobs & Icalc & \multicolumn{1}{l}{ Io-c } \\
& & & & \\
1 & a & 445.57807 & 445.57471 & 0.00336 \\
1 & b & 956.46485 & 956.46416 & 0.00069 \\
1 & c & 1058.51411 & 1058.51388 & 0.00023 \\
2 & a & 447.61120 & 447.61409 & -0.00288 \\
2 & b & 985.88522 & 985.88732 & -0.00210 \\
2 & c & 1089.15775 & 1089.15639 & 0.00136 \\
3 & a & 451.09110 & 451.09360 & -0.00251 \\
3 & b & 974.87261 & 974.87072 & 0.00189 \\
3 & c & 1081.92149 & 1081.92450 & -0.00300 \\
4 & a & 453.49753 & 453.49543 & 0.00211 \\
4 & b & 1003.53926 & 1003.53973 & -0.00047 \\
4 & c & 1112.15516 & 1112.15377 & 0.00140
\end{tabular}

\begin{tabular}{rrr}
\multicolumn{1}{c}{ Bobs } & \multicolumn{1}{l}{ Bcalc } & \multicolumn{1}{l}{ Bo-c } \\
& & \\
1134.2098 & 1134.2183 & -0.0085 \\
528.3822 & 528.3826 & -0.0004 \\
477.4419 & 477.4420 & -0.0001 \\
1129.0580 & 1129.0507 & 0.0073 \\
512.6145 & 512.6134 & 0.0011 \\
464.0090 & 464.0096 & -0.0006 \\
1120.3480 & 1120.3418 & 0.0062 \\
518.4052 & 518.4062 & -0.0010 \\
467.1125 & 467.1112 & 0.0013 \\
1114.4030 & 1114.4082 & -0.0052 \\
503.5967 & 503.5964 & 0.0002 \\
454.4141 & 454.4147 & -0.0006
\end{tabular}

Correlation coefficients:

$\begin{array}{lrrrrrrrr} & & 1 & 2 & 3 & 4 & 5 & 6 & 7 \\ \text { 1: } & \mathrm{R}(28,1) & 1.000 & & & & & & \\ 2: & \mathrm{R}(31,28) & -0.937 & 1.000 & & & & & \\ 3: & \mathrm{A}(28,1,2) & -0.971 & 0.912 & 1.000 & & & & \\ 4: & \mathrm{A}(31,28,1) & 0.549 & -0.736 & -0.411 & 1.000 & & & \\ 5: & \mathrm{D}(28,1,2,4) & -0.174 & 0.381 & -0.007 & -0.901 & 1.000 & & \\ 6: & \mathrm{D}(31,28,1,2) & 0.402 & -0.594 & -0.235 & 0.969 & -0.967 & 1.000 & \\ 7: & \mathrm{A}(29,28,1) & 0.034 & -0.229 & -0.160 & 0.044 & 0.045 & 0.045 & 1.000\end{array}$

Principal coordinates and estimated uncertainties:

$\begin{array}{crcrcrc}\text { ATOM NO. } & \mathrm{A} & \mathrm{dA} & \mathrm{B} & \mathrm{dB} & \mathrm{C} & \mathrm{dC} \\ & & & & & & \\ 1 & -1.23577 & 0.00080 & -1.58058 & 0.00126 & 0.32221 & 0.00142 \\ 2 & -0.42549 & 0.00048 & -0.74346 & 0.00091 & -0.01122 & 0.00114 \\ 3 & 0.85955 & 0.00086 & -0.35483 & 0.00068 & 0.73871 & 0.00020 \\ 4 & -0.47814 & 0.00160 & 0.13669 & 0.00198 & -1.26226 & 0.00207 \\ 5 & 1.28534 & 0.00187 & -1.37515 & 0.00189 & 1.78490 & 0.00176 \\ 6 & 0.80895 & 0.00110 & 0.97586 & 0.00115 & -1.10574 & 0.00117 \\ 7 & 1.79226 & 0.00059 & -0.03271 & 0.00048 & -0.44773 & 0.00113\end{array}$



$\begin{array}{ll}0.51080 & 0.00232\end{array}$
$\begin{array}{ll}1.05351 & 0.00109\end{array}$
$\begin{array}{ll}1.30277 & 0.00164\end{array}$
$0.54281 \quad 0.00140$
$\begin{array}{ll}1.97820 & 0.00022\end{array}$
0.041990 .00250
$\begin{array}{lll}3.13326 & 0.00039\end{array}$
$0.58943 \quad 0.00147$
$\begin{array}{ll}-0.03662 & 0.00231\end{array}$
$\begin{array}{ll}2.10123 & 0.00242\end{array}$
$\begin{array}{ll}-1.26112 & 0.00103\end{array}$
$\begin{array}{ll}0.49223 & 0.00277\end{array}$
$\begin{array}{ll}-1.51043 & 0.00227\end{array}$
$\begin{array}{ll}-1.32683 & 0.00274\end{array}$
$1.47419 \quad 0.00131$
$-2.34976 \quad 0.00155$
$\begin{array}{lll}2.52376 & 0.00084\end{array}$
$\begin{array}{ll}2.19001 & 0.00265\end{array}$
$\begin{array}{ll}-1.04719 & 0.00286\end{array}$
$1.17026 \quad 0.00213$
$1.42895 \quad 0.00190$
$\begin{array}{ll}1.32834 & 0.00297\end{array}$
$2.30337 \quad 0.00265$
$\begin{array}{ll}-0.47630 & 0.00309\end{array}$
$\begin{array}{ll}-0.49961 & 0.00281\end{array}$
$\begin{array}{ll}-1.39796 & 0.00139\end{array}$
$\begin{array}{ll}0.72506 & 0.00255\end{array}$
$1.31917 \quad 0.00199$
$2.73975 \quad 0.00036$
$\begin{array}{ll}-0.40386 & 0.00160\end{array}$
$\begin{array}{ll}2.49705 & 0.00097\end{array}$
$\begin{array}{lll}-0.45138 & 0.00301\end{array}$
$\begin{array}{ll}1.06905 & 0.00136\end{array}$
$-2.03045 \quad 0.00113$
$\begin{array}{ll}-2.14966 & 0.00145\end{array}$
$\begin{array}{lll}-1.27819 & 0.00387\end{array}$
$0.12453 \quad 0.00221$
$\begin{array}{ll}1.27164 & 0.00343\end{array}$
$1.33356 \quad 0.00213$
$3.68563 \quad 0.00091$
$0.90272 \quad 0.00148$
$\begin{array}{ll}3.74735 & 0.00061\end{array}$
$\begin{array}{ll}-0.14712 & 0.00224\end{array}$
$\begin{array}{ll}-0.11715 & 0.00427\end{array}$
$\begin{array}{ll}1.81949 & 0.00294\end{array}$
$2.03458 \quad 0.00090$
$\begin{array}{ll}-0.92768 & 0.00275\end{array}$
$\begin{array}{ll}3.02768 & 0.00169\end{array}$
1.458340 .00186
$\begin{array}{ll}2.81089 & 0.00217\end{array}$
$\begin{array}{ll}-1.91819 & 0.00101\end{array}$
$0.48894 \quad 0.00385$
$\begin{array}{ll}2.55846 & 0.00348\end{array}$
$\begin{array}{ll}-0.94787 & 0.00177\end{array}$
0.612510 .00133
$1.21874 \quad 0.00307$
$\begin{array}{ll}-1.86005 & 0.00143\end{array}$
$\begin{array}{ll}-0.81786 & 0.00433\end{array}$
$\begin{array}{lll}-2.26976 & 0.00304\end{array}$
$\begin{array}{ll}-3.81757 & 0.00018\end{array}$
$\begin{array}{ll}-0.92995 & 0.00058\end{array}$
$\begin{array}{ll}-1.56164 & 0.00215\end{array}$
$\begin{array}{ll}-3.13566 & 0.00287\end{array}$
$\begin{array}{ll}-1.61215 & 0.00294\end{array}$
$\begin{array}{ll}-0.46904 & 0.00077\end{array}$
$\begin{array}{ll}-4.59164 & 0.00112\end{array}$
$\begin{array}{ll}-1.33842 & 0.00283\end{array}$
$\begin{array}{ll}-0.44042 & 0.00220\end{array}$
$\begin{array}{ll}-3.00897 & 0.00022\end{array}$
$\begin{array}{ll}1.65544 & 0.00037\end{array}$
$\begin{array}{ll}-0.06260 & 0.00107\end{array}$
$0.37489 \quad 0.00040$
$\begin{array}{ll}-3.46253 & 0.00019\end{array}$
0.841150 .00029
$2.29910 \quad 0.00030$
0.12502
$0.50994 \quad 0.00092$
$\begin{array}{ll}-3.71515 & 0.00026\end{array}$ I
$0.50994 \quad 0.00092$ 1

NOTES: 1/ only the uncertainties for those coordinates which are completely defined by the fitted internals should be trusted

2/ the uncertainties are somewhat limited by the linear approximation coord $=(d$ coord/d parameter $) *$ parameter used for evaluation

3 / only the effect of the internals R, A, and D is propagated 
Table S36: The abbreviated results of the fit of the $r_{0}$ geometry of the complex of camphor with three water molecules $(3 \mathrm{w})$ to 24 rotational constants from 8 different isotopic species.

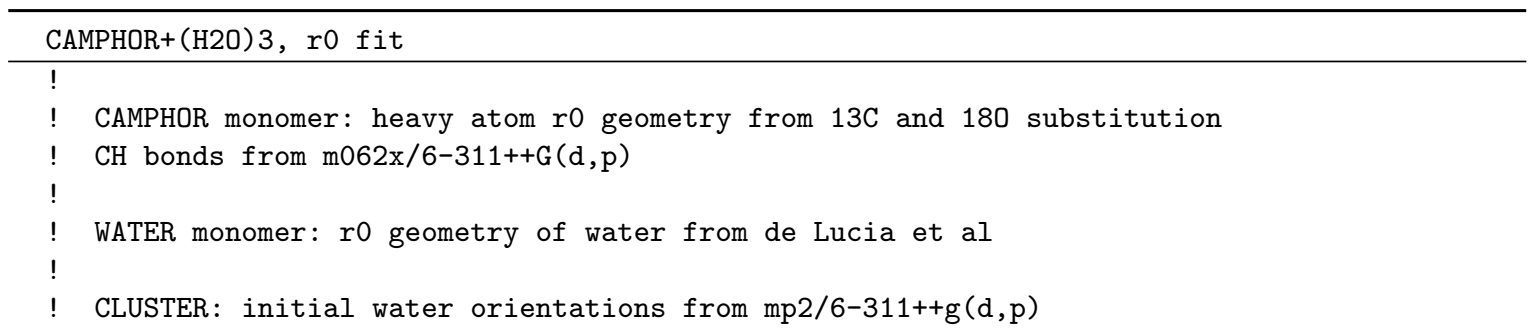
NO NA NB NC NO.NA NO.NA.NB NO.NA.NB.NC

$\begin{array}{rrrrrrrr}1 & 0 & 0 & 0 & 0.000000 & 0.000000 & 0.000000 & 15.9949150 \\ 2 & 1 & 0 & 0 & 1.211820 & 0.000000 & 0.000000 & 12.0000000 \\ 3 & 2 & 1 & 0 & 1.537772 & 126.809199 & 0.000000 & 12.0000000 \\ 4 & 2 & 1 & 3 & 1.530530 & 126.809199 & -180.000000 & 12.0000000 \\ 5 & 3 & 2 & 1 & 1.522123 & 113.549876 & 16.282273 & 12.0000000 \\ 6 & 4 & 2 & 5 & 1.544440 & 101.592660 & -13.450329 & 12.0000000 \\ 7 & 3 & 2 & 1 & 1.543159 & 100.538201 & 145.707425 & 12.0000000 \\ 8 & 3 & 2 & 4 & 1.556672 & 102.599801 & 71.743276 & 12.0000000 \\ 9 & 6 & 4 & 2 & 1.546869 & 106.492954 & -72.338525 & 12.0000000 \\ 10 & 7 & 3 & 5 & 1.534400 & 114.009223 & -62.945723 & 12.0000000 \\ 11 & 7 & 3 & 5 & 1.541843 & 113.152398 & 60.314627 & 12.0000000 \\ 12 & 5 & 3 & 7 & 1.092362 & 110.169968 & -177.564593 & 1.0078250 \\ 13 & 5 & 3 & 7 & 1.092696 & 111.042046 & -58.810315 & 1.0078250 \\ 14 & 5 & 3 & 7 & 1.093066 & 110.880759 & 62.313034 & 1.0078250 \\ 15 & 6 & 7 & 3 & 1.091300 & 114.744054 & -179.991580 & 1.0078250 \\ 16 & 4 & 2 & 1 & 1.091947 & 109.214947 & -60.264332 & 1.0078250 \\ 17 & 4 & 2 & 1 & 1.092014 & 110.532763 & 58.015422 & 1.0078250 \\ 18 & 9 & 8 & 3 & 1.090643 & 111.495730 & -117.151534 & 1.0078250 \\ 19 & 9 & 8 & 3 & 1.091195 & 112.393614 & 122.383106 & 1.0078250 \\ 20 & 8 & 9 & 6 & 1.092260 & 113.017512 & -118.338281 & 1.0078250 \\ 21 & 8 & 9 & 6 & 1.092177 & 111.982622 & 120.675625 & 1.0078250 \\ 22 & 10 & 7 & 3 & 1.094181 & 109.821977 & -173.038088 & 1.0078250 \\ 23 & 10 & 7 & 3 & 1.093540 & 110.269998 & 68.672957 & 1.0078250 \\ 24 & 10 & 7 & 3 & 1.089732 & 113.467178 & -52.324402 & 1.0078250 \\ 25 & 11 & 7 & 3 & 1.092891 & 110.095853 & -74.815171 & 1.0078250 \\ 26 & 11 & 7 & 3 & 1.093755 & 110.312134 & 166.591559 & 1.0078250 \\ 27 & 11 & 7 & 3 & 1.092074 & 113.427837 & 45.253198 & 1.0078250 \\ 28 & 1 & 2 & 4 & 2.764097 & 118.571881 & -5.174111 & 15.9949150 \\ 29 & 28 & 1 & 2 & 0.965000 & 3.747889 & 140.330834 & 1.0078250 \\ 30 & 28 & 29 & 1 & 0.965000 & 104.780000 & -150.991061 & 1.0078250 \\ 31 & 28 & 1 & 2 & 2.746954 & 99.884587 & -57.811250 & 15.9949150 \\ 32 & 31 & 28 & 1 & 0.965000 & 6.504518 & -167.209620 & 1.0078250 \\ 33 & 31 & 32 & 28 & 0.965000 & 104.780000 & 119.186991 & 1.0078250 \\ 34 & 31 & 28 & 1 & 2.798593 & 102.187720 & -1.964741 & 15.9949150 \\ 35 & 34 & 31 & 28 & 0.965000 & 3.720893 & -161.433538 & 1.0078250 \\ 36 & 34 & 35 & 31 & 0.965000 & 104.780000 & -144.393303 & 1.0078250 \\ & & & & & & & \end{array}$

ISOTOPIC SPECIES 2, changes from parent species: atom no., parameter no., value $34 \quad 4 \quad 417.9991315$ !

$! \quad \mathrm{Ni}=3: \quad 02=28$

ISOTOPIC SPECIES 3, changes from parent species: atom no.,parameter no., value $\quad \begin{array}{llll}28 & 4 & 17.9991315\end{array}$ ! 
!

ISOTOPIC SPECIES 4, changes from parent species:

atom no.,parameter no., value $\begin{array}{llll}31 & 4 & 17.9991315\end{array}$

!

! $\quad \mathrm{Ni}=5: \quad 02+03=28+31$

ISOTOPIC SPECIES 5, changes from parent species:

atom no., parameter no., value $\quad \begin{array}{llll}28 & 4 & 17.9991315\end{array}$

atom no.,parameter no., value $\quad \begin{array}{lll}31 & 4 & 17.9991315\end{array}$

!

$! \quad \mathrm{Ni}=6: \quad 01+02=28+34$

ISOTOPIC SPECIES 6, changes from parent species:

atom no., parameter no., value $\quad \begin{array}{lll}28 & 4 & 17.9991315\end{array}$

atom no.,parameter no., value $\quad \begin{array}{lll}34 & 4 & 17.9991315\end{array}$

!

$! \quad \mathrm{Ni}=7: \quad 01+03=31+34$

ISOTOPIC SPECIES 7 , changes from parent species:

atom no., parameter no., value $\quad \begin{array}{lll}31 & 4 & 17.9991315\end{array}$

atom no., parameter no., value $\quad \begin{array}{lll}34 & 4 & 17.9991315\end{array}$

!

$! \quad \mathrm{Ni}=8: \quad 01+02+03=28+31+34$

ISOTOPIC SPECIES 8, changes from parent species:

atom no., parameter no., value $\quad \begin{array}{llll}28 & 4 & 17.9991315\end{array}$

atom no.,parameter no., value $\quad \begin{array}{lll}31 & 4 & 17.9991315\end{array}$

atom no.,parameter no., value $\quad \begin{array}{llll}34 & 4 & 17.9991315\end{array}$

converged after: 8 iterations, ALAMDA $=0.10 \mathrm{E}-10$

FINAL RESULTS OF LEAST SQUARES FIT:

\begin{tabular}{|c|c|c|c|c|}
\hline $\mathrm{R}(28,1)$ & & 2.804016 & +- & 0.005119 \\
\hline $\mathrm{R}(31,28)$ & $=$ & 2.761338 & +- & 0.002737 \\
\hline$R(34,31)$ & $=$ & 2.800836 & +- & 0.002783 \\
\hline $\mathrm{A}(28,1,2)$ & $=$ & 114.966264 & +- & 0.233450 \\
\hline$A(31,28,1)$ & $=$ & 100.586962 & +- & 0.112803 \\
\hline$A(34,31,28)$ & $=$ & 103.444023 & +- & 0.090519 \\
\hline$D(28,1,2,4)$ & $=$ & -0.787286 & +- & 0.681056 \\
\hline$D(31,28,1,2)$ & $=$ & -52.727535 & +- & 0.167530 \\
\hline$D(34,31,28,1)$ & $=$ & -10.512289 & +- & 0.216204 \\
\hline $\mathrm{D}(33,31,32,28)$ & $=$ & 154.438948 & +- & 1.953760 \\
\hline \multicolumn{3}{|c|}{ Chi-squared $=$} & \multicolumn{2}{|c|}{0.0034587391} \\
\hline Devi & of & Eit $=$ & \multicolumn{2}{|c|}{0.015718} \\
\hline
\end{tabular}

\begin{tabular}{|c|c|c|c|c|c|c|c|}
\hline $\mathrm{Ni}$ & Axis & Iobs & Icalc & Io-c & Bobs & Bcalc & $\mathrm{Bo}-\mathrm{C}$ \\
\hline 1 & a & 565.77908 & 565.77591 & 0.00316 & 893.2444 & 893.2494 & -0.0050 \\
\hline 1 & $\mathrm{~b}$ & 1259.95176 & 1259.93193 & 0.01983 & 401.1098 & 401.1161 & -0.0063 \\
\hline 1 & c & 1418.50072 & 1418.51920 & -0.01848 & 356.2769 & 356.2722 & 0.0046 \\
\hline 2 & a & 575.90361 & 575.90919 & -0.00559 & 877.5410 & 877.5325 & 0.0085 \\
\hline 2 & $\mathrm{~b}$ & 1272.23716 & 1272.23432 & 0.00283 & 397.2365 & 397.2374 & -0.0009 \\
\hline 2 & c & 1438.81497 & 1438.82555 & -0.01058 & 351.2467 & 351.2441 & 0.0026 \\
\hline 3 & a & 573.71041 & 573.72299 & -0.01258 & 880.8957 & 880.8763 & 0.0193 \\
\hline 3 & $\mathrm{~b}$ & 1281.00975 & 1281.00066 & 0.00909 & 394.5161 & 394.5189 & -0.0028 \\
\hline 3 & c & 1447.36511 & 1447.36714 & -0.00202 & 349.1717 & 349.1713 & 0.0005 \\
\hline 4 & a & 567.18298 & 567.16117 & 0.02181 & 891.0335 & 891.0677 & -0.0343 \\
\hline 4 & $\mathrm{~b}$ & 1295.26407 & 1295.26061 & 0.00346 & 390.1745 & 390.1755 & -0.0010 \\
\hline
\end{tabular}




$\begin{array}{rrrrrrrr}4 & \mathrm{c} & 1453.56838 & 1453.57405 & -0.00566 & 347.6816 & 347.6803 & 0.0014 \\ 5 & \mathrm{a} & 575.30814 & 575.30130 & 0.00684 & 878.4493 & 878.4597 & -0.0104 \\ 5 & \mathrm{~b} & 1315.63865 & 1315.65089 & -0.01225 & 384.1321 & 384.1285 & 0.0036 \\ 5 & \mathrm{c} & 1481.96322 & 1481.96260 & 0.00062 & 341.0199 & 341.0201 & -0.0001 \\ 6 & \mathrm{a} & 584.38953 & 584.41308 & -0.02355 & 864.7982 & 864.7633 & 0.0348 \\ 6 & \mathrm{~b} & 1292.61242 & 1292.61644 & -0.00403 & 390.9749 & 390.9737 & 0.0012 \\ 6 & \mathrm{c} & 1467.57516 & 1467.57269 & 0.00247 & 344.3633 & 344.3639 & -0.0006 \\ 7 & \mathrm{a} & 577.21978 & 577.20617 & 0.01361 & 875.5400 & 875.5607 & -0.0206 \\ 7 & \mathrm{~b} & 1307.34037 & 1307.34278 & -0.00242 & 386.5703 & 386.5696 & 0.0007 \\ 7 & \mathrm{c} & 1473.41081 & 1473.39673 & 0.01408 & 342.9994 & 343.0027 & -0.0033 \\ 8 & \mathrm{a} & 585.87021 & 585.87344 & -0.00324 & 862.6126 & 862.6078 & 0.0048 \\ 8 & \mathrm{~b} & 1327.06935 & 1327.08543 & -0.01608 & 380.8234 & 380.8187 & 0.0046 \\ 8 & \mathrm{c} & 1501.71290 & 1501.69376 & 0.01913 & 336.5350 & 336.5393 & -0.0043\end{array}$

Principal coordinates and estimated uncertainties:

\begin{tabular}{|c|c|c|c|c|c|c|}
\hline ATOM NO. & A & $\mathrm{dA}$ & B & $d B$ & C & $\mathrm{dC}$ \\
\hline 1 & -0.75560 & 0.00428 & -1.46503 & 0.00624 & -0.97760 & 0.00475 \\
\hline 2 & -0.04370 & 0.00221 & -0.71143 & 0.00524 & -0.35007 & 0.00404 \\
\hline 3 & 1.35372 & 0.00350 & -0.21311 & 0.00479 & -0.75457 & 0.00132 \\
\hline 4 & -0.35712 & 0.00543 & -0.06687 & 0.01395 & 1.00228 & 0.00881 \\
\hline 5 & 1.99638 & 0.00634 & -1.03700 & 0.01421 & -1.86139 & 0.00920 \\
\hline 6 & 0.91744 & 0.00371 & 0.76936 & 0.00845 & 1.25034 & 0.00633 \\
\hline 7 & 2.03130 & 0.00365 & -0.11944 & 0.00182 & 0.62871 & 0.00449 \\
\hline 8 & 1.09071 & 0.01300 & 1.27760 & 0.00543 & -1.11766 & 0.00819 \\
\hline 9 & 0.85747 & 0.00995 & 1.96312 & 0.00343 & 0.26844 & 0.01315 \\
\hline 10 & 3.41283 & 0.00175 & 0.54773 & 0.00907 & 0.60329 & 0.00869 \\
\hline 11 & 2.19030 & 0.01444 & -1.48851 & 0.00305 & 1.31982 & 0.01268 \\
\hline 12 & 1.36482 & 0.01093 & -1.02820 & 0.01577 & -2.75263 & 0.00583 \\
\hline 13 & 2.11407 & 0.00596 & -2.07967 & 0.01318 & -1.55646 & 0.01549 \\
\hline 14 & 2.97716 & 0.00915 & -0.63438 & 0.02091 & -2.12740 & 0.01234 \\
\hline 15 & 1.08257 & 0.00791 & 1.04608 & 0.01329 & 2.29298 & 0.00697 \\
\hline 16 & -0.51375 & 0.01337 & -0.85064 & 0.01879 & 1.74627 & 0.00588 \\
\hline 17 & -1.27504 & 0.00286 & 0.52164 & 0.01865 & 0.94252 & 0.01686 \\
\hline 18 & 1.61845 & 0.01318 & 2.71670 & 0.00104 & 0.47469 & 0.01338 \\
\hline 19 & -0.11307 & 0.01239 & 2.45814 & 0.00906 & 0.32933 & 0.02096 \\
\hline 20 & 0.24697 & 0.01723 & 1.39762 & 0.00522 & -1.80085 & 0.01330 \\
\hline 21 & 1.97285 & 0.01776 & 1.67292 & 0.01281 & -1.62599 & 0.00600 \\
\hline 22 & 3.77489 & 0.00570 & 0.69030 & 0.00667 & 1.62594 & 0.00996 \\
\hline 23 & 4.13300 & 0.00057 & -0.09196 & 0.01558 & 0.08561 & 0.01581 \\
\hline 24 & 3.41646 & 0.00822 & 1.51946 & 0.01151 & 0.11010 & 0.00475 \\
\hline 25 & 2.99926 & 0.01508 & -2.05422 & 0.00573 & 0.85082 & 0.02005 \\
\hline 26 & 2.44899 & 0.01932 & -1.35392 & 0.00720 & 2.37398 & 0.01333 \\
\hline 27 & 1.29217 & 0.01750 & -2.10768 & 0.00661 & 1.26856 & 0.01137 \\
\hline 28 & -3.23285 & 0.00072 & -2.01966 & 0.00114 & 0.21325 & 0.00301 \\
\hline 29 & -2.37734 & 0.00111 & -1.88943 & 0.00209 & -0.21380 & 0.00222 \\
\hline 30 & -3.64560 & 0.00131 & -2.74094 & 0.00079 & -0.27728 & 0.00485 \\
\hline 31 & -4.16531 & 0.00055 & 0.54069 & 0.00271 & 0.66057 & 0.00367 \\
\hline 32 & -3.94111 & 0.00051 & -0.38825 & 0.00253 & 0.52627 & 0.00173 \\
\hline 33 & -4.92085 & 0.01908 & 0.52731 & 0.00378 & 1.26074 & 0.02466 \\
\hline 34 & -2.36233 & 0.00103 & 2.14745 & 0.00109 & -0.75797 & 0.00287 \\
\hline 35 & -3.02350 & 0.00111 & 1.64211 & 0.00102 & -0.26939 & 0.00223 \\
\hline 36 & -2.87215 & 0.00150 & 2.69727 & 0.00152 & -1.36543 & 0.00320 \\
\hline
\end{tabular}

NOTES: 1/ only the uncertainties for those coordinates which are completely defined by the fitted internals should be trusted

2/ the uncertainties are somewhat limited by the linear approximation coord $=(\mathrm{d}$ coord/d parameter $) *$ parameter used for evaluation

3 / only the effect of the internals R, A, and D is propagated 